\title{
Breaking GUT groups in F-theory
}

\author{
Ron Donagi ${ }^{1}$ and Martijn Wijnholt ${ }^{2}$
}

${ }^{1}$ Department of Mathematics, University of Pennsylvania, Philadelphia, PA 19104-6395, USA

${ }^{2}$ Max Planck Institute (Albert Einstein Institute), Am Mühlenberg 1, D-14476 Potsdam-Golm, Germany

\begin{abstract}
We consider the possibility of breaking the GUT group to the Standard Model gauge group in $F$-theory compactifications by turning on certain $U(1)$ fluxes. We show that the requirement of massless hypercharge is equivalent to a topological constraint on the UV completion of the local model. The possibility of this mechanism is intrinsic to $F$-theory. We address some of the phenomenological signatures of this scenario. We show that our models predict monopoles as in conventional GUT models. We discuss in detail the leading threshold corrections to the gauge kinetic terms and their effect on unification. They turn out to be related to Ray-Singer torsion. We also discuss the issue of proton decay in Ftheory models and explain how to engineer models which satisfy current experimental bounds.
\end{abstract}

\section{Contents}

1 Introduction

2 Breaking the GUT group

e-print archive: http://lanl.arXiv.org/abs/0808.2223 
2.1 The basic proposal 1528

2.2 More refined version

1532

2.3 Other comments 1536

2.3.1 Doublet-triplet splitting 1536

2.3.2 No heterotic or $M$-theory duals 1537

3 GUT monopoles 1537

4 Precision of unification: threshold corrections

1540

4.1 Leading corrections to gauge kinetic terms 1540

4.2 One-loop KK thresholds

1544

4.2.1 Generalities

1544

4.2.2 KK modes of $S U(5)$ gauge fields

1546

4.2.3 Anomaly and dependence on $\Lambda$

1550

4.2.4 KK modes of matter fields

4.3 Toy models

1558

4.4 Conclusions 1563

5 Proton decay 1565

5.1 Fibered root systems and their monodromies 1565

5.2 Extra $U(1)$ 's and their D-terms 1572

5.3 Proton decay operators of dimension four and five

5.4 Operators of dimension six 1583

5.5 Conclusions 1588

Acknowledgments 1589 
$\begin{array}{ll}\text { Appendix } & \text { A GUT breaking fluxes constructed } \\ \text { through the cylinder map }\end{array}$

Appendix B Metric anomaly for holomorphic torsion

Appendix C Roots of $E_{8}$

References
1590

1594

1596

1598

\section{Introduction}

One of the most remarkable hints about physics at high energy scales is the apparent unification of gauge couplings $[1,2]$. This gives strong support for the idea that the Standard Model originates from a single GUT group at some high scale [3]. Several other properties of the Standard Model point to some kind of unification; however, there are also aspects that are hard to fit in a conventional four-dimensional GUT model, particularly the doublet-triplet splitting problem. As has been appreciated for some time, the discrepancies have natural resolutions if the unification takes place not in four but in higher dimensions $[4,5]$. Of course gauge theories in higher dimensions do not make sense by themselves, but they have a natural home in string theory, which provides their UV completion.

The first models of this type were explored in the heterotic string, where unification takes place in ten dimensions [6]. However the experimental fact of the hierarchy between the $\mathrm{TeV}$ scale and the Planck scale, as well as practical considerations, have led to the idea of local model building [7-10]. In this scenario, one considers models of particle physics where the effects of four-dimensional gravity (and its attendant complications) may be treated as a small perturbation. That is, a local model is defined by the existence of a decoupling limit [10]

$$
g_{Y M}^{2}(\mu) \text { fixed, } \quad M_{P l} / \mu \rightarrow \infty .
$$

In the context of GUT models, this means we would fix the coupling at the GUT scale $\mu \sim M_{\mathrm{GUT}}$, and we will have at least two independent expansion parameters, $\alpha_{\mathrm{GUT}}$ and $M_{\mathrm{GUT}} / M_{P l}$.

Although perhaps not apparent at first sight, insisting on the twin principles of unification and local model building cuts down the landscape of possibilities considerably. In particular one might have thought that models 
Table 1: Branes with exceptional gauge symmetry in string theory.

\begin{tabular}{ll}
\hline Dim & Stringy realization \\
\hline 10d & $E_{8} \times E_{8}$ heterotic string \\
9d & strongly coupled type I' \\
8d & $F$-theory on ALE \\
7d & $M$-theory on ALE \\
6d & IIa on ALE/IIb with NS5 \\
\hline
\end{tabular}

with $D$-branes provide a natural realization of these principles. Unfortunately attempts to construct GUTs in D-brane models ran into fundamental difficulties, due to the absence of matter in the spinor representation of $S O(10)$, or the perturbative vanishing of top quark Yukawa couplings for $S U(5)$ (since the epsilon tensor needed for these couplings cannot be generated in perturbative open string theory). These problems can be traced back to the absence of exceptional gauge symmetries on perturbative $D$-branes. GUT groups can of course be naturally incorporated in the $E_{8} \times E_{8}$ heterotic string, however the ideas about local model building and decoupling from the Planck scale leads one to ask if exceptional Yang-Mills symmetries can also be localized on branes with non-zero codimension.

Apart from the heterotic string, exceptional Yang-Mills theory can in fact also appear on various branes in string theory (see table 1). However Yang-Mills theories realized on 5-branes or branes of lower dimension do not lead to chiral matter, because if the codimension is high enough then we can always separate the branes. On the other hand, if the codimension is too small, one gets into trouble with the requirement of decoupling. The situation in type I' should be very similar to the situation in Horava-Witten theory, to which it is related by compactification on a circle. For a generic compactification in the Horava-Witten set-up, if the size of the interval gets too large then the bare coupling of one of the $E_{8}$ s is pushed to infinity, which yields a bound of the form [11]

$$
G_{N} \gtrsim \frac{\alpha_{\mathrm{GUT}}^{2}}{M_{\mathrm{GUT}}^{2}},
$$

This means that $M_{\mathrm{GUT}} / M_{P l}$ is never parametrically small and the gravitational back-reaction may not be considered subleading. Another issue for the strongly coupled type I' is that there are no known constructive techniques. A similar issue affects the study of $7 d$ Yang-Mills theory in $M$-theory. This requires the study of compactification on 7 -manifolds of $G_{2}$ holonomy, a 
subject about which so little is known that we cannot even write equations for semi-realistic local models. ${ }^{1}$

Thus, if one is interested in a framework for local model building with GUT groups, on second thought it turns out the options are really rather limited. In $F$-theory, like in the heterotic string, there are actually powerful constructive techniques available from algebraic geometry [13,14]. Up until recently, the main issue hindering progress in this direction was the lack of knowledge about engineering chiral matter, an issue that was pointed out and solved in [15] by turning on fluxes (see also [16], and further refinement in [17]). However, although GUT groups and chiral matter could be naturally obtained in this framework, there was not yet a sound method for breaking the GUT group to the Standard Model while preserving the main predictions from unification.

The main purpose this paper is to make such a proposal. As we discuss in Section 2, this can be achieved by turning on suitable $U(1)$ fluxes. There are however a number of subtleties in the implementation. In particular, analogous mechanisms are not available in $M$-theory on $G_{2}$ or the heterotic string. This is an additional motivation to study unification in $F$-theory.

Having specified a GUT breaking mechanism, we can now start to address some of the phenomenology of $F$-theoretic GUTs. There are many issues one could discuss, and we focus on only a few of them. This paper is a direct continuation of [15], so we refer to that paper for basic concepts and notation.

In Section 3, we show that our models predict monopoles carrying magnetic hypercharge. They correspond to certain D3-branes ending on the GUT 7-brane.

In Section 4, we give a detailed discussion of threshold corrections to the gauge kinetic terms. It is well known that the GUT prediction of $\alpha_{3}\left(M_{Z}\right)$ differs slightly from the experimental value, and the natural question is if the heavy threshold corrections in our scenario make the situation worse or better. We will see that at least in our toy models, the corrections come with varying signs and can make the discrepancy worse or better. We will also see that the Kaluza-Klein (KK) scale ends up parametrically lower than the the GUT scale, although in practice this is a mild effect.

In Section 5, we discuss proton decay. Dimension four and five operators violating baryon number are generically present and models have to be

\footnotetext{
${ }^{1}$ Since this paper first appeared, the construction of local $M$-theory models has been described in [12].
} 
engineered to guarantee their absence. We find that decay through dimension six operators is parametrically enhanced as $\alpha_{\mathrm{GUT}} \rightarrow 0$ compared to four-dimensional models. A similar situation had earlier been discovered in other KK models of unification [18,19]; however, the parametric dependence for the $p \rightarrow \pi^{0} e_{L}^{+}$channel is different from what was seen in these papers. Moreover a second effect, the lowering of the KK scale compared to the GUT scale, also did not appear in $M$-theory.

Note for revision: The present version of this paper includes significant clarifications and improvements over the original version arXiv:0808.2223. Most of these were designed to bring the paper more in line with the subsequent papers $[22,70]$. We also extended the discussion of extra light $Z^{\prime}$ s, expanded the discussion of flux quantization and exotic matter in Appen$\operatorname{dix}$ A, and added Appendix C. As this paper was being prepared for submission, two papers appeared $[20,21]$ that overlap with some of our results.

\section{Breaking the GUT group}

\subsection{The basic proposal}

We start with a brief recap of the discussion in [15]. There are basically three ideas for breaking the GUT group in string models. The first is to engineer an adjoint Higgs or a Higgs in another large representation. Although this is in principle possible in $F$-theory, in this way one ends up with a traditional four-dimensional GUT model. Such models are beset by phenomenological problems such as doublet/triplet splitting and a tension between fast proton decay and unification. Therefore it is more desirable to use the additional ingredients in our $F$-theoretic constructions and to look for an intrinsically higher dimensional mechanism which preserves the successes of unification but evades the problems.

The second approach, which has been successfully implemented in the heterotic string, is to use discrete Wilson lines. However in order to avoid adjoint Higgses in F-theory, we would typically wrap our 7-brane on fourcycles with trivial fundamental group ${ }^{2}$. Attempts to dualize heterotic models with discrete Wilson lines lead to 7-branes with singularities on the worldvolume. Although the presence of singularities is common in recent phenomenological literature on extra-dimensional GUTs $[25,26]$, in the present

\footnotetext{
${ }^{2}$ One could consider smooth models with discrete Wilson lines, based on the Enriques surface or some surface of general type. However, an application of the Riemann-Roch formula shows that such models have light lepto-quarks.
} 
context we should avoid them. There could very well be new degrees of freedom at such singularities that spoil the running of the couplings or are not mutually supersymmetric with the other ingredients in the construction, or give rise to light particles with fractional electric charges as happens in analogous heterotic orbifold constructions. Given our current understanding of $F$-theory, it is impossible to know. Until this point is clarified, this approach does not appear promising in the context of $F$-theory.

The third idea is to turn on $U(1)$ fluxes on the worldvolume of the 7-brane in order to break the GUT group. For definiteness let us consider the case of a 7-brane with a GUT group $G=S U(5)$ wrapping a four-cycle $S$. We denote the elliptically fibered Calabi-Yau four-fold by $Y_{4}$, the section by $\sigma\left(B_{3}\right)$ and the inclusion of $S$ by $i: S \rightarrow \sigma\left(B_{3}\right)$. Then in order to break the GUT group we could turn on a non-trivial flux on $S$ for the hypercharge gauge field, which is embedded in $S U(5)$ as

$$
Y=\left(\begin{array}{ccccc}
-1 / 3 & 0 & 0 & 0 & 0 \\
0 & -1 / 3 & 0 & 0 & 0 \\
0 & 0 & -1 / 3 & 0 & 0 \\
0 & 0 & 0 & 1 / 2 & 0 \\
0 & 0 & 0 & 0 & 1 / 2
\end{array}\right)
$$

This means we specify a line bundle $L$ on $S$, with $c_{1}(L)$ a non-trivial class in $H^{2}(S)$ represented by a harmonic two-form, and set the $S U(5)$ field strength to $\left\langle F_{S U(5)}\right\rangle=c_{1}(L) Y$. More precisely, recall that abelian gauge fields descend from harmonic two-forms in the lattice

$$
\Lambda=\left\{\omega \in H^{2}\left(Y_{4}\right) \mid \omega \cdot \gamma=0 \text {, when } \gamma \in H_{2}\left(B_{3}\right) \text { or } \gamma=\left[T^{2}\right]\right\} .
$$

Let us denote the harmonic two-form leading to the hypercharge gauge field by $\omega_{Y}$ :

$$
C^{(3)}=A_{Y} \wedge \omega^{Y}
$$

Then let us turn on a $G$-flux ${ }^{3}$

$$
\mathrm{G} / 2 \pi=c_{1}(L) \wedge \omega^{Y}
$$

More generally, we would consider a global Calabi-Yau four-fold with a $G$-flux, which reproduces our local model with the above $G$-flux in a degeneration limit. The two-form $\omega^{Y}$ is somewhat singular, it has a delta-function

\footnotetext{
${ }^{3}$ Recall that the $G$-flux must be properly quantized, which means in the present case that the flux of the line bundle $L$ must be quantized. This results in a small puzzle regarding exotic matter, which we discuss and resolve in the next subsection.
} 
piece localized on the $A_{4}$ singularity giving rise to the $S U(5)$ gauge group. (To make sense of it mathematically, we should first resolve the $A_{4}$ singularity; $F$-theory corresponds to the limit when the exceptional cycles are taken to zero size.)

Such a $U(1)$ flux will break the GUT group $G=S U(5)$ to the subgroup which commutes with the $U(1)$, which is the Standard Model gauge group $S U(3) \times S U(2) \times U(1)$. Further we assume large volumes so that we can trust the predictions from the two derivative eight-dimensional Yang-Mills action. Then with this method of breaking the GUT group we recover the standard $S U(5)$ relations between the gauge couplings.

Unfortunately this argument is too quick, for two reasons. As we will discuss in the next subsection, naively turning on such a flux leads to leptoquarks in the low-energy spectrum, which is unacceptable. Actually, as we will show later this problem is naturally resolved by a more careful examination of the flux we should turn on, so we will not worry about this here. The second issue is that the Yang-Mills theory is coupled to additional closed string modes, which live in the bulk of spacetime, which may lead the abelian gauge fields to pick up a mass through the Stückelberg mechanism. The $F$-theory effective action has a Chern-Simons term of the form ${ }^{4}$

$$
\mathscr{L}_{12} \sim-\frac{i}{4 \pi} \int C_{4} \wedge \mathrm{G} \wedge \mathrm{G} .
$$

Now we expand the four-form as follows:

$$
C_{4}=C_{2}^{M} \wedge \beta_{M}, \quad \beta_{M} \in H^{2}\left(B_{3}\right)
$$

Note that these are bulk modes and know about all of $B_{3}$ (or more precisely, the section $\left.\sigma_{B_{3}}\right)$. We can expand the $G$-flux as

$$
\mathrm{G}=F_{Y} \wedge \omega^{Y}+G_{\text {internal }}
$$

where $\omega^{Y} \in \Lambda$ is the harmonic two-form giving rise to hypercharge.

\footnotetext{
${ }^{4}$ The reader might wonder why we do not consider couplings to other RR or NS potentials (we would like to thank J. Maldacena for asking the question). For instance, naively one should also take couplings like $\int C^{(2)} \wedge \operatorname{Tr}(F \wedge F \wedge F)$ into account. However, due to the $S l(2, Z)$ monodromies, in a generic $F$-theory compactification there are no zero modes for any of the RR or NS potentials with two indices on $R^{4}$, except for $C_{\mathrm{RR}}^{(4)}$. This is clear from the $M$-theory description.
} 
Then we find the following four-dimensional coupling:

$$
\mathscr{L}_{4} \sim-\frac{\mathrm{i}}{2 \pi} \Pi_{M}^{Y} \int d^{4} x C_{2}^{M} \wedge F_{Y}
$$

where

$$
\Pi_{M}^{Y}=\int_{Y_{4}} \beta_{M} \wedge \omega^{Y} \wedge G_{\text {internal }}
$$

As is well-known, the fields $C_{2}^{M}$ may be dualized to $\mathrm{RR}$ axions $a_{M}$ in four dimensions, and (2.8) then leads to a coupling of the form

$$
\mathscr{L}_{4} \sim \int d^{4} x G^{M N} g^{\mu \nu}\left(\Pi_{M} A_{Y, \mu}-\partial_{\mu} a_{M}\right)\left(\Pi_{N} A_{Y, \nu}-\partial_{\nu} a_{N}\right)
$$

where

$$
G_{M N}=\int_{B_{3}} \beta_{M} \wedge * \beta_{N}
$$

Next we plug in $\mathrm{G}_{\text {int }} / 2 \pi=c_{1}(L) \wedge \omega^{Y}$. In a local model (a $d P_{8}$ or $d P_{9}$ fibration over $S$ ), we may use $\int_{d P_{8}} \omega_{Y} \wedge \omega_{Y}=\operatorname{Tr}\left(Y^{2}\right)$, leading to

$$
\Pi_{M}^{Y}=-2 \pi \operatorname{Tr}\left[Y^{2}\right] \int_{S} c_{1}(L) \wedge i^{*} \beta_{M}
$$

Therefore at first sight it seems that $\Pi_{M}^{Y}$ must be non-zero for some $M$, and the hypercharge gauge field will pick up a mass through (2.8) or (2.10).

We would like to make a brief comment on our argument. We assumed that $\omega^{Y}$ can be represented by a class that is localized at the $S U(5)$ singularity, so that the overlap $\Pi_{M}^{Y}$ only depends on the pull-back $i^{*} \beta_{M}$ of $\beta_{M}$ to $S$. This could hardly be otherwise, because we would expect that for a $U(1)$ gauge symmetry that is part of an unbroken non-abelian group, the abelian and non-abelian components are localized in the same way, so it should be in the singularity lattice of the $d P_{9}$ surface. We will see this explicitly for hypercharge below. For abelian gauge symmetries that are not part of a non-abelian group, there is no such localization and no guarantee that the corresponding $\omega$ can be extended globally.

One way to avoid the conclusion that hypercharge is lifted, suggested in [27], is to turn on a flux in the same cohomology class $\left[c_{1}(L)\right]$ in a hidden sector. The axion will then couple to a linear combination of $A_{\mu}^{Y}$ and a $U(1)$ in the hidden sector, and the orthogonal linear combination will remain 
massless. But in this approach the kinetic term of the remaining massless $U(1)$ is changed compared to our original hypercharge generator, and we lose the relation $\sqrt{5 / 3} g_{1}=g_{2}=g_{3}$.

Here we would like to propose a different way out, which has the virtue of preserving the GUT relations among the couplings at leading order. The situation we discussed above is very analogous to that of obtaining a massless hypercharge in models with branes at singularities [28]. In fact in [29], Section 6 , the mechanism of [28] was already interpreted as a partial unification scenario. The point is that because the brane has non-zero codimension, the vanishing of $\Pi_{M}^{Y}$ for all $M$ does not imply that $c_{1}(L)$ must vanish. Rather it is equivalent to the following statement. If we embed $i: S \rightarrow B_{3}$ then

$$
i^{*}: H^{2}\left(B_{3}\right) \rightarrow H^{2}(S)
$$

generally has non-trivial cokernel, and $\Pi_{M}^{Y}=0$ for all $M$ means that $c_{1}(L)$ is a generator of this cokernel. Thus the requirement of a massless hypercharge gauge field would really amount to a topological constraint on the UV completion of the local model.

It is useful to restate this criterion using Poincaré duality. Consider the two-cycle $\Xi \in H_{2}(S)$ dual to $c_{1}(L)$. Then the requirement $\Pi_{M}^{Y}=0$ for all $M$ is equivalent to the statement that $\Xi$ is in the kernel of the map $i: H_{2}(S) \rightarrow$ $H_{2}\left(B_{3}\right)$. In other words, even though $\Xi$ is a non-trivial two-cycle in $S$, once we embed it in $B_{3}$ we can find a three-chain $\Gamma$ in $B_{3}$ so that $\Xi=\partial \Gamma$.

One quick observation is that the canonical divisor $K_{S}$ is always a pullback of a class in $H^{2}\left(B_{3}\right)$. This follows from the adjunction formula, and the fact that $K_{B_{3}}$ and $\mathcal{O}(S)$ yield classes in $H^{2}\left(B_{3}\right)$. So in any local construction we should certainly make sure that $c_{1}(L)$ is orthogonal to $c_{1}\left(K_{S}\right)$. Generically we would expect there is one other class in the image of $i^{*}$, namely the remaining linear combination of $c_{1}\left(K_{B_{3}}\right)$ and $c_{1}(\mathcal{O}(S))$ (which may be proportional to $c_{1}\left(K_{S}\right)$ however $)$, and the remaining classes to be orthogonal to two-forms inherited from $B_{3}$. This is because divisors in the local Picard group typically get destroyed when higher order terms are added to the equations in order to compactify the model [28]. However for special UV completions the set of inherited classes may be larger.

\subsection{More refined version}

We now deal with the other problem briefly mentioned above. The issue is that due to quantization constraints on the flux for $A_{Y}$, naively it seems that turning on such a flux leads to massless lepto-quarks, which is 
phenomenologically unacceptable. Here by lepto-quarks we mean chiral fields with the same gauge quantum numbers as the $X$ and $Y$ gauge bosons of conventional $S U(5)_{\text {GUT }}$ models. To see this, consider the decomposition of the $S U(5)$ adjoint under $S U(3) \times S U(2) \times U(1)_{Y}$ :

$$
\begin{aligned}
\mathbf{2 4} & =(\mathbf{3}, \mathbf{1})_{0} \oplus(\mathbf{1}, \mathbf{8})_{0} \oplus(\mathbf{1}, \mathbf{1})_{0} \oplus(\mathbf{2}, \mathbf{3})_{-\mathbf{5} / \mathbf{6}} \oplus(\mathbf{2}, \overline{\mathbf{3}})_{\mathbf{5} / \mathbf{6}} \\
& \equiv \mathbf{R}_{0} \oplus \mathbf{R}_{-5 / 6} \oplus \mathbf{R}_{5 / 6} .
\end{aligned}
$$

In order to get the MSSM at low energies, we cannot have any massless modes in the representation $\mathbf{R}_{5 / 6}$. This means in particular that

$$
\chi\left(S, L^{5 / 6}\right)=0
$$

Since $\operatorname{deg}(L)=c_{1}(L) \cdot K_{S}=0$ as noted above, and since we take $S$ to be a Del Pezzo surface in order to avoid massless adjoint valued fields, we have

$$
\chi\left(S, L^{5 / 6}\right)=1+\frac{1}{2} c_{1}\left(L^{5 / 6}\right)^{2}
$$

from the Hirzebruch-Riemann-Roch formula. So the absence of leptoquarks requires that $c_{1}\left(L^{5 / 6}\right)^{2}=-2$. On the other hand, since

$$
\begin{aligned}
10 & =(2,3)_{1 / 6}+(1,1)_{1}+(1, \overline{3})_{-2 / 3} \\
\overline{\mathbf{5}} & =(\mathbf{2}, \mathbf{1})_{-1 / 2}+(\mathbf{1}, \overline{\mathbf{3}})_{1 / 3}
\end{aligned}
$$

it may naively seem that we also need $c_{1}\left(L^{1 / 6}\right)$ and $c_{1}\left(L^{1 / 3}\right)$ to be integer classes, so that $L^{5 / 6}, L^{1 / 6}, L^{1 / 3}$ are all honest line bundles on $S$. But this requirement is inconsistent with $c_{1}\left(L^{5 / 6}\right)^{2}=-2$, so it seems we would not get any sensible models without extra lepto-quarks this way.

This issue arose because we tried to apply the quantization constraints the hypercharge flux and the flux for generating chiral matter separately. However the correct thing to do is to apply the constraints only to the total flux. We will now analyze the model in more detail and see that quantization only requires $L^{5 / 6}$ to be an honest line bundle, and is therefore perfectly consistent with the absence of massless lepto-quarks.

Consider a local model for an $S U(5)$ GUT obtained from unfolding an $E_{8}$ singularity. That is we consider an $E_{8}$ ALE fibered over $S_{2}$, with the vanishing cycles labeled by the roots of $E_{8}$ (see figure 1). We take $\left\{\alpha_{-\theta}, \alpha_{1}, \alpha_{2}, \alpha_{3}, \alpha_{4}\right\}$ to be of finite volume and varying over $S_{2}$, and we take the monodromy for $\left\{\alpha_{5}, \ldots, \alpha_{8}\right\}$ to be trivial. This breaks the gauge group from $E_{8}$ to $S U(5)$. The first group of cycles may undergo monodromies corresponding to Weyl reflections generated by $\left\{\alpha_{-\theta}, \alpha_{1}, \alpha_{2}, \alpha_{3}\right\}$. These Weyl 


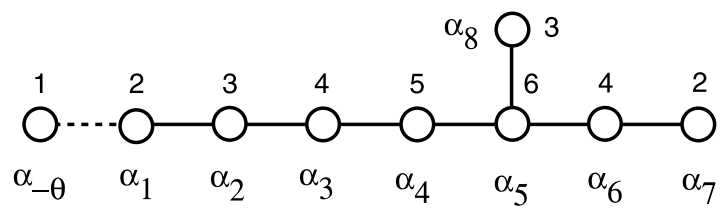

Figure 1: The extended $E_{8}$ Dynkin diagram and Dynkin indices.

reflections leave the second group of cycles invariant. We denote the corresponding Weyl group as $\mathscr{W}_{A_{4}}$.

When the two-cycle $\alpha_{4}$ in the ALE (or some $\mathscr{W}_{A_{4}}$ image) shrinks to zero size, the singularity increases to $S O(10)$, so this corresponds to the matter curve $\Sigma_{\mathbf{1 0}}$. Similarly, when the two-cycle $\alpha_{3}+\alpha_{4}$ (or some $\mathscr{W}_{A_{4}}$ image) shrinks to zero size, we get the matter curve $\Sigma_{\mathbf{5}}$.

Let us define a canonical basis of Cartan generators $\omega_{i}$ satisfying

$$
\left[\omega_{i}, \alpha_{j}\right]=\delta_{i j}
$$

Let us write $\omega_{Y}$ in this basis, taking the roots $\left\{\alpha_{6}, \alpha_{7}\right\}$ and $\left\{\alpha_{8}\right\}$ in the Dynkin diagram to correspond to the roots of $S U(3)_{c} \times S U(2)_{w}$ respectively. Naively one might think that the hypercharge generator should be identified with $\omega_{Y}=-5 / 6 \omega_{5}$. However, this generator assigns a non-zero $U(1)$ charge to $\alpha_{-\theta}$, hence it does not commute with the $\mathscr{W}_{A_{4}}$ monodromy group and receives a mass of order the KK scale. Furthermore it gives incorrect hypercharge assignments to the hypermultiplets, so we should really not call this generator hypercharge. Since we know the hypercharge assignments of the hypermultiplets on the matter curves and of the $S U(5)$ gauge bosons, we see that the correct hypercharge generator $\omega_{Y}$ must satisfy

$$
\left[\omega_{Y}, \alpha_{5}\right]=-\frac{5}{6}, \quad\left[\omega_{Y}, \alpha_{4}\right]=1
$$

and all the remaining commutators zero. Note that these equations are consistent in that the equation $\left[\omega_{Y}, \alpha_{-\theta}\right]=0$ actually follows from the definition of $\alpha_{\theta}$ and the remaining commutators. Hence, we deduce

$$
\omega_{Y}=-\frac{5}{6} \omega_{5}+\omega_{4}
$$

Dually we may also represent this by a linear combination of algebraic cycles on the $d P_{9}$ (fibered over the base), and we find

$$
\omega_{Y} \simeq \frac{1}{6}\left(2 \alpha_{7}+4 \alpha_{6}+6 \alpha_{5}+3 \alpha_{8}\right)
$$


This is indeed a linear combination of effective cycles in the singularity lattice, as expected for a $U(1)$ which is part of an unbroken non-abelian gauge symmetry. Turning on a flux for this $U(1)_{Y}$ breaks the $S U(5)$ group to $S U(3) \times S U(2) \times U(1)$. Furthermore, this generator actually commutes with the $\mathscr{W}_{A_{4}}$ Weyl group that breaks the $E_{8}$ to $S U(5)_{\text {GUT }}$; in particular, it is defined globally over $S$ and defines an unbroken $U(1)$ gauge symmetry.

As discussed in [15], in $F$-theory the Cartan generators $\omega_{i}$ correspond to two-forms on the ALE, which we denote by the same name, and the $\alpha_{j}$ correspond to two-cycles of the ALE. The integral of the $\omega_{i}$ yield the $U(1)$ charges of of BPS membranes (or rather $(p, q)$-strings) wrapped on the corresponding two-cycle, in particular with our definition we have $\int_{\alpha_{j}} \omega_{i}=$ $\delta_{i j}$ so the periods of the $\omega_{i}$ are all integral. Now turning on a flux for the $U(1)$ gauge field associated to $\omega_{Y}$, corresponds to turning on an additional G- flux of the form

$$
\frac{\mathrm{G}_{Y}}{2 \pi}=c_{1}(L) \wedge \omega_{Y}=-c_{1}\left(L^{5 / 6}\right) \wedge \omega_{5}+c_{1}(L) \wedge \omega_{4},
$$

where $L$ is a (possibly fractional) line bundle on $S$. The last piece looks problematic for satisfying the quantization conditions. However since the last piece commutes with the generators of the $S U(5)_{\text {GUT }}$ group, it can be canceled by including a similar fractionally quantized piece with opposite sign in the flux we turn on to generate chiral matter. Hence for the total flux to be properly quantized, only $c_{1}\left(L^{5 / 6}\right)$ and its multiples need to be integer quantized, but not $c_{1}(L)$ or $c_{1}\left(L^{1 / 6}\right)$. As we already noted, this is perfectly compatible with the requirement of the absence of massless leptoquarks.

In this way of stating it, it also becomes clear that the same freedom should exist in spectral cover constructions for the heterotic string. What we have discussed is the $F$-theory dual of heterotic models with structure groups of the form $S(U(n) \times U(1)) \subset S U(n+1)$ [30-33], where for us the relevant case is $n=5$. See [15] for the relevant aspects of heterotic/ $F$-theory duality.

In Appendix A we discuss how to write down the fluxes in the heterotic language, and their dual $G$-flux constructed using the projected cylinder map, which gives a precise algebro-geometric definition of (2.22). We also show in more detail there that the quantization constraint is compatible with the absence of light exotic matter, taking into account the issue of ramification (which we did not treat carefully above). To compare with our description here, recall that the sheets of the spectral cover are labelled by generators of the adjoint representation of $E_{8}$. For $S(U(5) \times U(1)) \subset S U(6)$, 
the most important piece is a six-fold cover whose sheets are labelled by the six roots

$$
\begin{array}{ll}
\sigma_{1}=\alpha_{5}, & \sigma_{4}=\alpha_{5}+\alpha_{4}+\alpha_{3}+\alpha_{2}, \\
\sigma_{2}=\alpha_{5}+\alpha_{4}, & \sigma_{5}=\alpha_{5}+\alpha_{4}+\alpha_{3}+\alpha_{2}+\alpha_{1}, \\
\sigma_{3}=\alpha_{5}+\alpha_{4}+\alpha_{3}, & \sigma_{6}=\alpha_{5}+\alpha_{4}+\alpha_{3}+\alpha_{2}+\alpha_{1}+\alpha_{-\theta} .
\end{array}
$$

This spectral cover is naturally reducible under the action of $\mathscr{W}_{A_{4}}$ - it splits into a copy of the zero section, labelled by $\left\{\alpha_{5}\right\}$, and a five-fold spectral cover whose sheets are labelled by $\left\{\sigma_{2}, \ldots, \sigma_{6}\right\}$. Since $\omega_{Y}$ evaluates to $1 / 6$ on each of the sheets of the five-fold cover, the $G$-flux $c_{1}(L) \wedge \omega_{Y}$ maps to a line bundle on this five-fold spectral cover which is morally $L^{1 / 6}=\left(L^{5 / 6}\right)^{1 / 5}$ on each sheet, i.e., it is a $U(5)$ spectral cover rather than an $S U(5)$ spectral cover. Similarly this $G$-flux yields a line bundle $L^{-5 / 6}$ on $\sigma_{1}$, the zero-section proportional to the hypercharge generator of $S U(5)_{\text {GUT }}$. One should keep in mind though that we only want part of a heterotic model (the observable sector, i.e., the local neighborhood of the GUT brane in F-theory language) while we would like to replace the hidden sector by something else. Indeed as we will see in the next subsection, $F$-theory models which implement our GUT breaking mechanism cannot have global heterotic duals.

The hypercharge $G$-flux defined through the cylinder map depends on the choice of the line bundle $L^{5 / 6}$ on $S_{2}$, which we henceforth call $\zeta$. In order to eliminate the axion couplings, $c_{1}(\zeta)$ must be orthogonal to any class inherited from $B_{3}$, in particular $c_{1}\left(K_{S}\right)$ and $c_{1}\left(N_{S}\right)$. For primitiveness, $\zeta$ must be orthogonal to the pull-back to $S$ of the Kähler class of $B_{3}$. This is also clearly guaranteed by the previous condition. Finally, in order to eliminate the light $(\mathbf{2}, \mathbf{3})_{-\mathbf{5} / \mathbf{6}}$ exotics from the spectrum, from (2.16) we see that $c_{1}(\zeta)$ must be one of the "simple roots", i.e., a cohomology class with $c_{1}(\zeta)^{2}=-2$ and $c_{1}(\zeta) \cdot K_{S}=0$. It is not hard to make toy models with three generations and no exotic matter; in Appendix A we explain how to do this using the cylinder map.

\subsection{Other comments}

\subsubsection{Doublet-triplet splitting}

It may be useful to point out that our GUT breaking mechanism does not lead to a doublet-triplet splitting problem. The zero modes for the doublets and triplets come from different Dolbeault cohomology groups, so we can have light doublets without having light triplets. This story is essentially the same as for GUT breaking with discrete Wilson lines: because there is never a four-dimensional $S U(5)$ gauge group at any scale, there is no 
need for four-dimensional triplet partners of the minimal supersymmetric standard model (MSSM) Higgses. Since there is still an eight-dimensional GUT group, there will of course still be KK modes with the same quantum numbers as the triplets, with masses of order the radius of curvature of the surface $S$, and they can be an issue for proton decay and precision unification. We discuss this in more detail in Sections 4 and 5.

\subsubsection{No heterotic or $M$-theory duals}

If $B_{3}$ is fibered over $S$, then the map $i^{*}: H^{2}\left(B_{3}\right) \rightarrow H^{2}(S)$ is automatically surjective. This is easy to see because any class $c_{1}(\zeta) \in H^{2}(S)$ may be pulled back to $B_{3}$. For such compactifications, our GUT breaking mechanism can therefore not be implemented. In particular, it does not work for duals of perturbative heterotic models, which correspond to $\mathbf{P}^{1}$ fibrations over $S$. In fact it cannot be implemented in the perturbative heterotic string at all, whether or not it has an $F$-theory dual, because the zero modes of the axions and gauge fields are both supported on all of the heterotic CalabiYau three-fold. One may still use the trick of getting a massless hypercharge as a linear combination of two different sectors, as studied in [30-33], though at the cost of giving up unification at leading order.

One might also consider implementing this mechanism in $M$-theory on manifolds of $G_{2}$ holonomy. Local models in this context consist of ALE fibrations over a compact three-manifold $Q$. In order to avoid adjoint Higgses one needs $b_{1}(Q)=0$. But this implies that $b_{2}(Q)=0$ also and so we cannot turn on any fluxes.

Thus, the possibility of breaking the GUT group by fluxes is intrinsic to $F$-theory and should contribute to distinctive phenomenological signatures.

\section{GUT monopoles}

In GUT models we expect to find solitons, particularly monopoles, which will be generated in the early universe through the Kibble mechanism (for a review, see [34]). This is true even for the higher dimensional models here; in a sense the role of the Higgs field is played by the internal components of eight-dimensional $U(1)$ gauge fields breaking the GUT group. For the analgous situation in the heterotic string, see [35].

Let us review quickly some aspects of four-dimensional GUT monopoles. If the GUT group $G$ is broken to a subgroup $H$, monopoles are classified by $\pi_{2}(G / H)$. From the long exact sequence

$$
\cdots \rightarrow \pi_{2}(G) \rightarrow \pi_{2}(G / H) \rightarrow \pi_{1}(H) \rightarrow \pi_{1}(G) \rightarrow \cdots
$$


and the fact that for any compact simple Lie group we have $\pi_{2}(G)=0$, we find that $\pi_{2}(G / H)=\pi_{1}(H)$. In the case of adjoint breaking of $G=S U(5)$ to the SM, we have

$$
H=[S U(3) \times S U(2) \times U(1)] / Z_{6}
$$

The action of $Z_{6}$ on $S U(3) \times S U(2)$ is identified with the center of $S U(3) \times$ $S U(2)$. Thus there exists a monopole with charge $1 / 2 g_{1}$. The monopole also carries $Z_{3}$ color and $Z_{2} S U(2)$ magnetic charge.

Now let us try to find the monopoles in our $F$-theory set-up. The important feature which allowed us to break the GUT group without mixing with other fields was the existence of an extra two-cycle on the worldvolume of the 7-brane, which becomes the boundary of a three-cycle when embedded in space-time. So we expect the existence of hypercharged monopoles must be related to this feature. Let us denote by $\Xi \in H_{2}(S)$ the Poincaré dual of $c_{1}(\zeta)$ in $S$. Since $c_{1}(\zeta)$ was automatically orthogonal to the Kähler class, then if $S$ is non-singular $\Xi$ can not be represented by a single geometric two-cycle in $S$, only by a linear combination of them. For definiteness let us say that $\Xi=A_{1}-A_{2}$ where $A_{1}$ and $A_{2}$ are geometric cycles (in particular they do not become boundaries when embedded in $B_{3}$ ).

Since our mechanism requires that $P D(\alpha)$ becomes a boundary when embedded in $B_{3}$, there exists a three-chain $\Gamma$ in $B_{3}$ such that

$$
A_{1}-A_{2}=\partial \Gamma
$$

Now we can wrap a $D 3$-brane on $\Gamma$. There must be a representative of $\Gamma$ with minimal volume greater than zero, because if we restrict to a sufficiently small neighborhood of the brane we know that $\Gamma$ does not exist. This is similar to a D3-ending-on-D5 system and so should correspond to the sought-after monopole.

Let us consider a small "six-sphere" surrounding the end of a stack of D3branes. The integral of $d F_{\mathrm{RR}}^{(5)}$ over any such six-sphere must vanish, but we get a contribution +1 for every open $D 3$-brane in the stack, as it intersects the sphere precisely once. However due to the Chern-Simons terms in the action, we also get contributions to $d F_{\mathrm{RR}}^{(5)}$ from other fluxes. Thus we can relate the flux through the sphere to the number of open $D 3$-branes.

Let $D$ be a divisor in $S_{2}$ such that $D \cdot\left[A_{1}-A_{2}\right]=1$. Such a divisor is guaranteed to exist by Poincaré duality. In the present case, we can take our "six-sphere" to correspond to a unit three-ball in $R^{3}$ times $D$ times a unit circle in the normal bundle to $S_{2}$ in $B_{3}$, minus a unit two-sphere in $R^{3}$ 
times $D$ times the unit disk in the normal bundle to $S_{2}$ in $B_{3}$. We have to take a linear combination, because otherwise the boundary is non-empty and the integral of $d F_{\mathrm{RR}}^{(5)}$ need not vanish. Our "six-sphere" intersects precisely once with each $D 3$-brane wrapped on $\Gamma$. Then the total flux through this "six-sphere" must be equal to the number $n$ of $D 3$ branes wrapped on $\Gamma$. More precisely, we consider the eight-manifold $T$, which consists of the "sixsphere" with the elliptic fibration on top of it. Then we must have

$$
N_{D 3}(\Gamma)=\frac{\chi(T)}{24}-\frac{1}{8 \pi^{2}} \int_{T} \mathrm{G} \wedge \mathrm{G}
$$

We will assume $\chi(T)=0$, which seems reasonable but which we have not derived. The four-form $\omega^{Y} \wedge \omega^{Y}$ is a delta-function on $Y_{4}$ localized on our 7-brane. The six-sphere intersects our 7-brane in a four-cycle, which is given by $S^{2} \times D$ with $\left[A_{1}-A_{2}\right] \cdot D=1$ and $S^{2}$ the unit two-sphere in $R^{3}$ surrounding the origin. The flux integral can be written as

$$
n=\frac{1}{2 \pi} \int_{S^{2}} F_{Y} \cdot \frac{1}{2 \pi} \int_{D} F_{Y} 2 \frac{1}{2} \operatorname{Tr}\left(Y^{2}\right) .
$$

Since $\frac{1}{2 \pi} \int_{D} F_{Y}=D \cdot\left[A_{1}-A_{2}\right]=1$, we see that the integral $\int_{S^{2}} F_{Y}$ is nonzero, and the particle obtained by wrapping a $D 3$ brane on $\Gamma$ carries magnetic hypercharge. It might be interesting to do the topological analysis carefully and see if the color $Z_{3}$ charge and the $S U(2) Z_{2}$ charge can be recovered also.

Our monopoles are also similar to those of $[36,37]$. The fact that the monopole is not supported on the GUT brane may seem somewhat puzzling, since one may expect that it can be understood as a bound state of the fields in the eight-dimensional gauge theory. A similar situation appears in the context of the $D 3$-ending-on- $D 5$ system, to which our configuration is similar. In that context, a field configuration on the $D 5$-brane satisfying the Nahm equations can be understood as a "fuzzy funnel", a spike growing out of the D5-brane. The two points of view have a slightly different range of validity.

In addition to monopoles, $F$-theory vacua have several other interesting types of solitons. There are cosmic strings from D3-branes wrapped on twocycles, and domain walls interpolating between vacua with different values of the $G$-flux. It should be interesting to examine their effect on early universe cosmology. 


\section{Precision of unification: threshold corrections}

\subsection{Leading corrections to gauge kinetic terms}

Even though we have engineered gauge coupling unification at leading order, we cannot expect unification to be precise, because there are many massive states charged under the Standard Model gauge group. The corrections to the leading terms at the GUT scale are organized in a power series in $\alpha_{\mathrm{GUT}}$, and we have

$$
\frac{16 \pi^{2}}{g_{a}^{2}\left(M_{\mathrm{GUT}}\right)}=\frac{4 \pi k_{a}}{\alpha_{\mathrm{GUT}}}+\delta_{a}^{(0)}+\delta_{a}^{(1 / 2)} \alpha_{\mathrm{GUT}}^{1 / 2}+\delta_{a}^{(1)} \alpha_{\mathrm{GUT}}+\cdots
$$

Such corrections are expected in any GUT model, for example in conventional $4 d$ GUTs one will get corrections due to the Higgs sector responsible for breaking the GUT group to the SM gauge group. In this section we would like to discuss in detail the leading threshold corrections, here denoted by $\delta_{a}^{(0)}$.

Actually there are two dimensionless parameters in our set-up, $\alpha_{\mathrm{GUT}}$ and $M_{\mathrm{GUT}} / M_{P l, 4}$. Since we assume the existence of a decoupling limit, we can make an expansion of the threshold corrections to the gauge kinetic terms:

$$
\delta \sim \delta_{\text {local }}+\frac{M_{\mathrm{GUT}}}{M_{P l}} \delta^{\prime}+\cdots .
$$

The $M_{p l}$ suppressed contributions can be regarded as sub-subleading. Thus we may focus on the charged states that remain dynamical in the local geometry.

The assumption of decoupling can fail because some corrections may become divergent if certain tadpoles are not canceled in the local model, but only globally. We will see how this can happen explicitly below. In fact it could happen that the tadpole is already canceled in a non-compact model which is slightly larger than the first infinitesimal neighborhood of the gauge brane, as was seen explicitly in [28]. In other words, it could be canceled already even when the four-dimensional Planck scale is infinite. To account for the general case, we will have to introduce a cut-off $\Lambda$ for the loop integrals, which in a concrete global model could be anywhere between the local KK scale and $M_{P l}$ a priori.

The charged states in the local geometry can be separated into two types. First, there are the ground states of open strings solitons which give us our $S U(5)$ gauge fields and $\mathbf{1 0}$ and $\overline{\mathbf{5}}$ matter. Even though the string scale is 
not parametrically separated from the $10 d$ Planck scale, these modes still have a weakly coupled description in terms of a higher dimensional gauge theory with enlarged gauge group containing $S U(5)$. They give a one-loop correction proportional to $\alpha_{\mathrm{GUT}}^{0}$, which may be expanded in KK harmonics, and they will be discussed at length in Section 4.2. The upshot will be that they are related to Ray-Singer torsion and can in principle be computed in any given model, even without knowing the Calabi-Yau metric. Second, there are the massive excitations of these same open strings solitons, the open strings which gave us the eight-dimensional gauge fields and $\mathbf{1 0}$ and $\overline{\mathbf{5}}$ matter. For finite $g_{s}$ they have masses of order the ten-dimensional Planck scale and were not included in our gauge theory. Integrating out such modes yields local higher derivative terms to the eight-dimensional gauge theory action, localized on $R^{4} \times S$ and $R^{4} \times \Sigma$. Since we do not have a weakly coupled description of these modes in $F$-theory, we cannot calculate the coefficients of such corrections, so we had better hope that they do not affect unification at order $\alpha_{\mathrm{GUT}}^{0}$. We will argue that this is indeed the case.

The leading higher derivative terms in eight dimensions that may affect unification are the $\operatorname{Tr}\left(F^{4}\right)$ and $\operatorname{Tr}\left(F^{2}\right)^{2}$ terms. Since they are local in eight dimensions and BPS protected, we may use $F$-theory/hetetotic duality to view these same corrections from the heterotic side, where they are wellstudied. Let us first consider the heterotic string on $T^{2}$, with Wilson lines turned on so that the gauge group is broken to $S U(5)$. It is believed that the $\operatorname{Tr}\left(F^{4}\right)$ and $\operatorname{Tr}\left(F^{2}\right)^{2}$ amplitudes are BPS saturated at one loop and receive no contributions beyond one-loop, up to non-perturbative corrections by $N S 5$-instantons. Now heterotic on $T^{2}$ is dual to $F$-theory on $K 3$, and we may take a low energy limit such that the heterotic string scale goes to infinity but the mass of the $S U(5) W$-bosons remains finite. In this limit, the $K 3$-surface degenerates to an $A_{4}$ ALE space. The only surviving BPS states in short multiplets are the $W$-bosons of $S U(5)$.

On the heterotic side, there are also tree level contributions to the $\operatorname{Tr}\left(F^{2}\right)^{2}$ terms. They come from ten dimensions as the gauge fields on the heterotic side live in ten dimensions. Upon compactifying to eight dimensions and mapping to $F$-theory, tree level $F^{4}$ terms are proportional to $V_{\mathbf{P}^{1}}^{-2}$ and one loop $F^{4}$ terms are independent of $V_{\mathbf{P}^{1}}$. The gauge kinetic terms are also independent of $V_{\mathbf{P}^{1}}$. Here $V_{\mathbf{P}^{1}}$ denotes the volume of the base of the $K 3$ surface. Therefore in the local limit considered here, the heterotic tree level $F^{4}$ terms decouple. We still get contributions at one loop, but only by short BPS multiplets. In our low energy limit, the only surviving BPS states are the $S U(5) W$-bosons, and so the amplitude degenerates to a straightforward one-loop amplitude in the $S U(5)$ gauge theory. (See e.g., [38] for a review of the analogous statements in six dimensions). In this limit, we find the 
following divergent contribution from the eight-dimensional supersymmetric Yang-Mills theory (SYM) [39,40]

$$
\log \left(\Lambda^{2} / \mu^{2}\right)\left[t_{8}+\epsilon_{8}\right] \operatorname{Tr}_{a d j}\left(F^{4}\right) .
$$

It is useful to express the trace in the adjoint in terms of fundamental traces using the identity (for $S U(n)$ gauge group)

$$
\operatorname{Tr}_{a d j}\left(F^{4}\right)=2 n \operatorname{Tr}_{f}\left(F^{4}\right)+6\left(\operatorname{Tr}_{f}\left(F^{2}\right)\right)^{2}
$$

Heterotic/type II duality predicts this to be the exact answer for the $F^{4}$ amplitude in the limit we are considering. (One can probably argue this more directly from the superspace structure of the $F^{4}$ terms).

From the perturbative type II perspective this may look somewhat strange, since we would expect a tree level $F^{4}$ contribution from the BornInfeld action, as well as loop corrections and non-perturbative corrections due to $D_{-1}$-instantons. This is not a contradiction, as this calculation is performed in a different weakly coupled frame. Since the amplitude is protected one should get the same answer either way after proper extrapolation, although clearly the full type II calculation (including $D$-instantons) is much more involved.

The above picture for the relation between the $F$-theory and IIb computations is confirmed in a number of instances where a comparison between heterotic and perturbative type II could be done explicitly. It was found that the heterotic one-loop result actually already contained and agreed with the type II result based on the DBI/Chern-Simons action in the weakly coupled type II limit $[41,43,44,87]$, and sums up further $D_{-1}$ instanton corrections to it. We expect that here too the heterotic result reproduces the full type II contribution in the IIb weak coupling limit. This is not completely unexpected, because the classical $F$-theory geometry sums up the $D_{-1}$ instanton corrections and so a one-loop calculation in an $F$-theory background should know about the instantons. It would be interesting to check this in more detail. ${ }^{5}$ Since the heterotic calculation is well-established, it is reasonable to assume the heterotic answer (really a one-loop field theory calculation) is the reliable result. ${ }^{6}$

\footnotetext{
${ }^{5}$ In light of the recent work [45] which shows that the Sen IIb limit is a generalization of the heterotic $S O(32)$ limit, old results about threshold corrections in heterotic/type I duality such as [86] are particularly relevant. The $D_{1}$-instantons in [86] correspond to $D_{-1^{-}}$ instantons in type IIb. Localization techniques for summing $D_{-1}$-instanton corrections have recently been discussed in [47].

${ }^{6}$ This revises a claim in v1 of this paper, where we added the DBI $F^{4}$ correction to the above one-loop amplitude. Since this DBI correction worsens a discrepancy with experimentally measured deviations from unification, this means that precision unification is in better shape than claimed in v1.
} 
Once we compactify and turn on a background flux, the above one-loop computation in the eight-dimensional gauge theory can be expanded in a sum over KK modes. This computation will be discussed in the next subsection, although we will use a more four-dimensional point of view there.

There could be further corrections to the effective action in eight dimensions at higher order in derivatives. However, they will be suppressed by additional powers of $\alpha_{\text {GUT }}$ and so are not of interest to us. Moreover, they should not affect the four-dimensional gauge kinetic terms at any rate because that would change the tension of a BPS D3 instanton configuration in the 7-brane, but not its charge.

So far we have discussed contributions from the strings whose ground states yield the $S U(5)$ gauge fields in eight dimensions. We further have to include the modes of the open strings stretching between different 7-branes, whose ground states give rise to charged matter in the $\mathbf{1 0}$ and $\overline{\mathbf{5}}$. The heterotic perspective seems less useful in this case, so we will argue more directly that the massive stringy tower cannot affect unification to the order we are considering.

Strings stretching between intersecting 7-branes could give rise to local higher derivative corrections of the form

$$
\int_{R^{4} \times \Sigma} \sqrt{g} F^{3} .
$$

The terms that can appear are constrained by $(5+1)$ dimensional Lorentz invariance. There could also be terms of type

$$
\int_{R^{4} \times \Sigma} \sqrt{g} F^{2} R,
$$

however upon reduction they would give an $S U(5)$ symmetric contribution to the gauge kinetic terms that can be absorbed in the bare coupling. Upon reduction of (4.5) to four dimensions, if non-vanishing the four-dimensional gauge kinetic terms can only be proportional to $\int_{\Sigma} F_{Y}$. However, we have already seen that for GUT breaking with fluxes to be viable, we must have $\int_{\Sigma} F_{Y}=0$. More precisely, this holds for the total matter curves $\Sigma_{\mathbf{5}}$ or $\Sigma_{\mathbf{1 0}}$. In practice these matter curves may be reducible, and $\int_{\Sigma_{i}} F_{Y} \neq 0$ for each irreducible piece. However, the contribution to a given gauge kinetic term is proportional only to the total flux through $\Sigma_{\mathbf{5}}$ and $\Sigma_{\mathbf{1 0}}$ :

$$
\sum_{i} \int_{\Sigma_{i}} F_{Y}=\int_{\Sigma_{\mathbf{5}, 1 \mathbf{0}}} F_{Y}=0,
$$


so this does not affect the argument that such corrections cannot affect unification. Therefore we have to go at least to the $F^{4}$ corrections on $R^{4} \times \Sigma$ to get a contribution that is not $S U(5)$ symmetric. However such higher derivative contributions will be proportional to at least $\alpha^{\prime} / R_{\mathrm{KK}}^{2} \sim \alpha_{\mathrm{GUT}}^{1 / 2}$ (i.e., they are suppressed by at least $\alpha_{\mathrm{GUT}}^{3 / 2}$ compared to the leading terms), so they are not relevant for our discussion as promised.

We have argued that in local models the leading corrections to unification come only from one-loop amplitudes of the gauge theory itself, i.e., from the eight-dimensional super-Yang-Mills multiplet and from the six-dimensional hypermultiplet matter. Thus it remains to expand these one-loop corrections as a sum over KK modes. This is the subject of the following subsections.

\subsection{One-loop KK thresholds}

We would now like to discuss loop corrections to unification from integrating out KK modes of the gauge theory. We will see that they can be determined without knowing the Calabi-Yau metric, and discuss the role of an anomaly for determining their moduli dependence.

\subsubsection{Generalities}

We briefly recall some relevant formulae. The one-loop running for the gauge couplings in four dimensions is given by

$$
\frac{16 \pi^{2}}{g_{a}^{2}(\mu)}=\frac{16 \pi^{2} k_{a}}{g^{2}}+b_{a} \log \left(\Lambda^{2} / \mu^{2}\right)+S_{a}
$$

with $\left(k_{1}, k_{2}, k_{3}\right)=(5 / 3,1,1)$. The one-loop beta-function coefficients are given by

$$
b_{a}=2 \operatorname{Tr}_{m=0}\left(Q_{a}^{2}(-1)^{F}\left(\frac{1}{12}-\chi^{2}\right)\right)
$$

where $Q_{a}$ is normalized so that $\operatorname{Tr}\left(Q_{a}^{2}\right)=k_{a} / 2$. In the MSSM, we have $\left(b_{1}, b_{2}, b_{3}\right)=(11,1,-3)$. Finally the threshold corrections are given by

$$
S_{a}=2 \operatorname{Tr}_{m \neq 0} Q_{a}^{2}(-1)^{F}\left(\frac{1}{12}-\chi^{2}\right) \log \left(\Lambda^{2} / m^{2}\right) .
$$

In our case this will be a sum over $N=1$ vector multiplets and chiral multiplets. The $\operatorname{Tr} Q_{a}^{2}$ piece in (4.9), (4.10) factors out for each supermultiplet, 
and we have

$$
\operatorname{Tr}(-1)^{F}\left(\frac{1}{12}-\chi^{2}\right)= \begin{cases}-3 / 2 & \text { (vector multiplet) } \\ 1 / 2 & \text { (chiral multiplet) }\end{cases}
$$

For later purposes we would like to expand (4.8) a little. If all matter would come in complete $S U(5)$ multiplets, then we would have $\left(b_{1}, b_{2}, b_{3}\right) \propto$ $\left(k_{1}, k_{2}, k_{3}\right)$. However the gauge multiplets and the electro-weak Higgses do not come in complete multiplets. Then we can decompose

$$
b_{a}=b_{a}^{(c)}+b_{a}^{(g)}+b_{a}^{(h)},
$$

where $b_{a}^{(g)}$ is the contribution from the $S U(3) \times S U(2) \times U(1)$ gauge fields, $b_{a}^{(h)}$ is the contribution from the Higgs doublets, and $b_{a}^{(c)}$ are the contributions from the remaining complete multiplets (the three generations of $\mathbf{1 0}$ and $\overline{\mathbf{5}}$ ). In other words we have

$$
b_{a}^{(g)}=(0,-6,-9), \quad b_{a}^{(h)}=(1,1,0), \quad b_{a}^{(c)}=(10,6,6) \propto\left(k_{1}, k_{2}, k_{3}\right) .
$$

To compute the one-loop threshold corrections, we need the massive spectrum of the Dirac operator of the eight-dimensional gauge theory. As explained in [15], after compactification this Dirac operator decomposes into various Dolbeault operators, coupled to holomorphic bundles. Let us recall some generalities. Consider a holomorphic bundle $V$ on $S$, and its associated Dolbeault operator

$$
\Omega_{S}^{0,0} \otimes R(V) \stackrel{\bar{\partial}}{\longrightarrow} \Omega_{S}^{0,1} \otimes R(V) \stackrel{\bar{\partial}}{\longrightarrow} \Omega_{S}^{0,2} \otimes R(V),
$$

where $R$ is some representation of the structure group of $V$. Let us denote $\mathrm{by}^{7}$

$$
\Delta_{n, R(V)}=\left(\bar{\partial}+\bar{\partial}^{\dagger}\right)^{2}=\bar{\partial} \bar{\partial}^{\dagger}+\bar{\partial}^{\dagger} \bar{\partial}
$$

the Laplacian acting on $\Omega_{S}^{0, n} \otimes R(V)$, where the adjoint is defined with respect to the Hermitian metric. Then we are interested in the spectrum of $\bar{\partial}+\bar{\partial}^{\dagger}$, or equivalently the spectrum of $\Delta_{n, R(V)}$ which gives the squares of the eigenvalues. More precisely, we are interested in the combination of eigenvalues appearing in (4.10). This is the logarithm of the determinant of

\footnotetext{
${ }^{7}$ Unfortunately this is not exactly the standard Laplacian, which differs by a factor of a two (i.e., $\Delta_{d}=2 \Delta_{\bar{\partial}}$ ). Since the squared masses of KK modes are conventionally defined to be the eigenvalues of $\Delta_{d}$, we should really replace the radius $R$ by $R / \sqrt{2}$ in all the formulae below, and similarly replace $M_{\mathrm{KK}}$ by $\sqrt{2} M_{\mathrm{KK}}$.
} 
$\Delta_{n, R(V)}$. Naively the sum over eigenvalues is not well defined. Traditionally it is defined using zeta function regularization [48]. That is we consider

$$
\zeta_{\Delta}(s)=\sum_{\lambda_{i}>0} \frac{1}{\lambda_{i}^{s}} .
$$

For elliptic operators one may show that $\zeta_{\Delta}(s)$ extends to a meromorphic function on the complex plane. Then we define

$$
\log \operatorname{det}^{\prime} \Delta=-\zeta_{\Delta}^{\prime}(0) \sim \log \prod \lambda_{i} .
$$

Here, we explicitly excluded the zero modes, because in (4.10) we want only the massive modes. This is indicated by the prime on the determinant.

We need to say a few words about the zeta-function regularization scheme used here. There are other regularization schemes for the sum over KK modes, see e.g., [49] for a discussion of some different schemes and their relations. The zeta-function regularization used in the definition of the holomorphic torsion is special in that it throws out power law divergences, and keeps only the logarithmic divergences. Power law divergences are however quite natural in a KK theory; in the present case they signal that the gauge coupling becomes dimensionful above the KK scale and has power law running. Thus the regularization provided by the microscopic theory underlying $F$-theory will certainly not be zeta-function regularization. ${ }^{8}$

However we have essentially already argued that the power law divergences cannot affect unification. We can also argue this directly; a term with power law divergence that could affect the relations between the $4 d$ gauge couplings must be of the schematic form

$$
\Lambda^{2} F^{3}
$$

on $S$. Since $g_{i \bar{j}} F_{Y}^{i \bar{j}}=0$ for the internal flux, such a term would have no effect on the $4 d$ gauge couplings.

\subsubsection{KK modes of $S U(5)$ gauge fields}

Now we are ready to write expressions for the one-loop contributions of KK modes to the gauge couplings. As discussed in [15] there are several scenarios we can consider. Here, we will take the case of an $S U(5)$ gauge field propagating in the bulk and all chiral matter localized on 7-brane intersections. It is not hard to write down analogous formal expressions for other scenarios,

\footnotetext{
${ }^{8}$ We would like to thank S. Raby for a conversation on this point.
} 
with one or more chiral fields and a larger gauge group supported in the bulk of the 7-brane (though of course evaluating them in explicit examples is another matter). Let us first consider the eight-dimensional $S U(5)$ gauge fields. Turning on a $U(1)$ flux proportional to hypercharge breaks $S U(5)$ to $S U(3) \times S U(2) \times U(1)$, and we decompose the adjoint representation of $S U(5)$ as

$$
\begin{aligned}
\mathbf{2 4} & =(\mathbf{3}, \mathbf{1})_{0} \oplus(\mathbf{1}, \mathbf{8})_{0} \oplus(\mathbf{1}, \mathbf{1})_{0} \oplus(\mathbf{2}, \mathbf{3})_{-\mathbf{5} / \mathbf{6}} \oplus(\mathbf{2}, \overline{\mathbf{3}})_{\mathbf{5} / \mathbf{6}} \\
& \equiv \mathbf{R}_{0} \oplus \mathbf{R}_{-5 / 6} \oplus \mathbf{R}_{5 / 6} .
\end{aligned}
$$

We would like to sum up the contributions of the KK modes of the $S U(5)$ gauge fields. For brevity we denote by $\Delta_{n, Y}$ the Laplacian acting on $\Omega^{0, n}\left(L^{Y}\right)$. Then for each eigen-vector of $\Delta_{0, Y}$ we get a vector multiplet in four dimensions in the representation $\mathbf{R}_{Y}$. Similarly for each eigenvector of $\Delta_{1, Y}$ we get a chiral multiplet, and for each eigenvector of $\Delta_{2, Y}$ we get an anti-chiral multiplet in the representation $\mathbf{R}_{Y}$, or equivalently a chiral multiplet in $\mathbf{R}_{Y}^{*}$. Note however that $\operatorname{Tr}_{\mathbf{R}_{Y}} Q_{a}^{2}=\operatorname{Tr}_{\mathbf{R}_{Y}^{*}} Q_{a}^{2}$. Thus we consider the linear combination

$$
\mathbf{K}_{Y}=\frac{3}{2} \log \operatorname{det}^{\prime} \frac{\Delta_{0, R(V)}}{\Lambda^{2}}-\frac{1}{2} \log \operatorname{det}^{\prime} \frac{\Delta_{1, R(V)}}{\Lambda^{2}}-\frac{1}{2} \log \operatorname{det}^{\prime} \frac{\Delta_{2, R(V)}}{\Lambda^{2}} .
$$

We also need

$$
\operatorname{Tr}_{\mathbf{R}_{0}}\left(Q_{a}^{2}\right)=(0,2,3), \quad \operatorname{Tr}_{\mathbf{R}_{5 / 6}}\left(Q_{a}^{2}\right)=(25 / 6,3 / 2,1) .
$$

Altogether then we find

$$
S_{a}^{g}=\sum_{Y=0, \pm 5 / 6} 2 \operatorname{Tr}_{\mathbf{R}_{Y}}\left(Q_{a}^{2}\right) \mathbf{K}_{Y}
$$

or more explicitly

$$
\begin{aligned}
& S_{1}^{g}=\frac{25}{3}\left(\mathbf{K}_{5 / 6}+\mathbf{K}_{-5 / 6}\right), \quad S_{2}^{g}=4 \mathbf{K}_{0}+3\left(\mathbf{K}_{5 / 6}+\mathbf{K}_{-5 / 6}\right), \\
& S_{3}^{g}=6 \mathbf{K}_{0}+2\left(\mathbf{K}_{5 / 6}+\mathbf{K}_{-5 / 6}\right)
\end{aligned}
$$

where $g$ indicates that these are the contributions from the gauge fields. Note incidentally that since $c_{1}\left(L^{5 / 6}\right)$ corresponds to a simple root, the Del Pezzo has a diffeomorphism symmetry (namely the Weyl reflection generated by $c_{1}\left(L^{5 / 6}\right)$ itself) which takes $L^{5 / 6} \rightarrow L^{-5 / 6}$, so we expect $\mathbf{K}_{5 / 6}$ and $\mathbf{K}_{-5 / 6}$ to be identical.

The expression we have written can be related to holomorphic Ray-Singer torsion. The appearance of Ray-Singer torsion is not unexpected; in the 
heterotic string [67] and in $M$-theory on manifolds of $G_{2}$ holonomy [18], the threshold corrections can also be related to Ray-Singer torsion. To see this, let us first note that the above vector and chiral multiplets pair up in a natural way into massive multiplets. We expand the linear fluctuations of the $8 d$ gauge field in terms of a complete set of eigenmodes of the Laplacian. In fact, it is convenient to look at the $(0,1)$ part of the gauge field with respect to a complex structure on $\mathbf{R}^{4} \times S_{2}$, so that the relevant Laplacian is given by $\Delta_{\bar{\partial}}=D^{2}$ :

$$
A_{8 d}=A_{c l}+\sum_{n} a_{(n) \mu} d x^{\mu} \wedge \psi_{(n)}^{0}+\sum_{m} \phi_{(m)} \psi_{(m)}^{1},
$$

where the zero-forms $\psi_{(n)}^{0}$ and the $(0,1)$-forms $\psi_{(m)}^{1}$ are eigenmodes. The $(1,0)$ part of the $8 d$ gauge field is recovered by Hermitian conjugation. In our application, $A_{c l}$ will be the hypercharge gauge field $A^{Y}$. Similarly, we expand the Higgs field

$$
\Phi_{8 d}=\Phi_{c l}+\sum_{m} \chi_{(m)} \psi_{(m)}^{2}
$$

where $\psi_{(m)}^{2}$ are $(0,2)$ forms which are eigenmodes of $\Delta_{\bar{\partial}}$. We will take $\Phi_{c l}=0$. In addition, the set of $(0, p)$ forms with values in $R(V)$ admits an orthogonal decomposition known as the Hodge decomposition:

$$
\Omega^{0, p}(R(V))=\mathbf{H} \oplus \mathrm{i} m(\bar{\partial}) \oplus \mathrm{i} m\left(\bar{\partial}^{\dagger}\right),
$$

where $\mathbf{H}$ are the zero modes (harmonic forms) of $\Delta_{\bar{\partial}}$. Since the Laplacian commutes with $\bar{\partial}$ and $\bar{\partial}^{\dagger}$, this decomposition is compatible with the decomposition in terms of eigenmodes of $\Delta_{\bar{\partial}}$.

Now, given an eigenmode $\psi_{(n)}^{0}$ of the Laplacian with eigenvalue $M_{(n)}^{2}$, we get another eigenmode with the same eigenvalue:

$$
\not \psi_{(n)}^{0}=\psi_{(n)}^{1}
$$

Similarly, given such an exact one-form $\psi_{(n)}^{1}$, by applying $\not D$ we recover the zero-form $\psi_{(n)}$. Furthermore, $\bar{\partial} \bar{\partial}^{\dagger}$ annihilates i $m\left(\bar{\partial}^{\dagger}\right)$. Therefore the massive spectrum of $\bar{\partial}^{\dagger} \bar{\partial}$ acting on zero-forms is the same as the massive spectrum of $\bar{\partial} \bar{\partial}^{\dagger}$ acting on $(0,1)$ forms.

This has a simple physical interpretation. Let us consider the effect of an eight-dimensional gauge transformation with parameter $\lambda$. Expanding 
$\delta A_{8 d}=\not D \lambda$ in KK modes, we see that the $4 d$ fields have the following transformation laws:

$$
\delta a^{(n)}=\bar{\partial} \lambda^{(n)}, \quad \delta \phi^{(n)}=\lambda^{(n)} .
$$

In particular, we find that $\phi^{(n)}$ describes the longitudinal part of a massive $4 d$ gauge field, whose \pm 1 polarizations are given by $a_{(n)}$. Thus the fact that the massive spectrum of $\bar{\partial}^{\dagger} \bar{\partial}$ acting on zero-forms is the same as the massive spectrum of $\bar{\partial} \bar{\partial}^{\dagger}$ acting on $(0,1)$ forms corresponds physically to the fact that a vector superfield eats a chiral field in order to obtain a mass. Similarly, the massive spectrum of $\bar{\partial}^{\dagger} \bar{\partial}$ acting on $(0,1)$-forms is the same as the massive spectrum of $\bar{\partial} \bar{\partial}^{\dagger}$ acing on $(0,2)$-forms. This corresponds to the fact that two chiral fields with opposite charges need to pair up in order to gain a mass.

Thus we may rewrite (4.20) as

$$
\mathbf{K}_{Y}=2 \log \operatorname{det}^{\prime} \frac{\Delta_{0, R(V)}}{\Lambda^{2}}-\log \operatorname{det}^{\prime} \frac{\Delta_{1, R(V)}}{\Lambda^{2}} .
$$

Now we define the holomorphic Ray-Singer torsion as

$$
\mathbf{T}_{R(V)}=\frac{1}{2} \sum_{k=0}^{2}(-1)^{k+1} k \log \operatorname{det}^{\prime} \Delta_{k, R(V)} .
$$

The determinants are defined as before using zeta-function regularization. Note that the conjugate linear $\bar{*}$-operator commutes with the Laplacian, and maps the eigenvectors and eigenvalues of $\Delta_{n, R(V)}$ to those of $\Delta_{2-n, R^{*}(V) \otimes K_{S}}$. Therefore we have

$$
\begin{aligned}
\mathbf{T}_{R(V)} & =\frac{1}{2}(-1)^{n+1} \sum_{k=0}^{n}(-1)^{n-k+1}(n-k) \log \operatorname{det}^{\prime} \Delta_{n-k, K \otimes R(V)^{*}} \\
& =(-1)^{n+1} \mathbf{T}_{R(V)^{*} \otimes K}
\end{aligned}
$$

and

$$
2 \mathbf{T}_{R^{*}(V) \otimes K_{S}}=-2 \log \operatorname{det}^{\prime} \Delta_{0, R(V)}+\log \operatorname{det}^{\prime} \Delta_{1, R(V)}
$$

is the the same as $\mathbf{K}_{Y}$, modulo the extra dependence on $\Lambda^{2}$. The relation between them can be found using

$$
\zeta_{\Delta / \Lambda^{2}}(s)=\Lambda^{2 s} \zeta_{\Delta}(s) \quad \Rightarrow \quad-\zeta_{\Delta / \Lambda^{2}}^{\prime}(0)=-\zeta_{\Delta}(0) \log \Lambda^{2}-\zeta_{\Delta}^{\prime}(0)
$$


Hence, we may write

$$
\mathbf{K}_{Y}=\mathbf{K}_{-Y}=\left.2 \mathbf{T}\left(S_{2}, L^{Y}\right)\right|_{R \rightarrow R \Lambda}
$$

where by $R=V_{S_{2}}^{1 / 4}$ we mean the scale of $S_{2}$.

\subsubsection{Anomaly and dependence on $\Lambda$}

In order to understand the dependence on the cutoff, we need to know the scaling dependence of the holomorphic torsion. Computing the torsion explicitly can quickly get somewhat complicated. But fortunately the metric dependence of the holomorphic torsion is determined by an anomaly (i.e., the torsion is a section of a non-trivial line bundle over the configuration space) and can be obtained rather easily. The anomalous variation of the torsion is described by the metric anomaly formula derived by Bismut et al., [51].

In Appendix B, we have worked out the dependence of the torsion on a rescaling of the metric, and hence the dependence on $\Lambda$. We find that

$$
\begin{aligned}
2 \mathbf{T}(X, V) \sim & {\left[\sum(-1)^{q}(2-q) h^{q}(X, V)-\int_{S}\left(c h_{2}(V)+\frac{5}{12} c_{1}(V) c_{1}(T)\right.\right.} \\
& \left.\left.+\frac{1}{24} c_{1}(T)^{2}+\frac{1}{12} c_{2}(T)\right)\right] \log R^{2} \Lambda^{2}
\end{aligned}
$$

Let us further rewrite the zero mode dependence as

$$
\begin{aligned}
& {\left[2 h^{0}(V)-h^{1}(V)\right] \log \left(R^{2} \Lambda^{2}\right)} \\
& \quad=\left[\frac{1}{2} \chi(V)-\left(-\frac{3}{2} h^{0}(V)+\frac{1}{2} h^{1}(V)+\frac{1}{2} h^{2}(V)\right)\right] \log \left(R^{2} \Lambda^{2}\right) .
\end{aligned}
$$

We also have the Riemann-Roch formula

$$
\chi(V)=\int_{S} \operatorname{ch}_{2}(V)+\frac{1}{2} c_{1}(V) c_{1}(T)+\frac{1}{12} c_{1}(T)^{2}+\frac{1}{12} c_{2}(T)
$$

where we may also recall that $\int_{S} c_{2}(T)=\chi(S)$. Then we may rewrite the contribution of the zero modes and the KK modes of the eight-dimensional 
gauge fields to the running as

$$
\begin{aligned}
\frac{16 \pi^{2}}{g_{a}^{2}(\mu)}= & \frac{16 \pi^{2} k_{a}}{g^{2}}+2 \sum_{Y=0, \pm \frac{5}{6}} \operatorname{Tr}_{R_{Y}}\left(Q_{a}^{2}\right)\left(-\frac{3}{2} h^{0}\left(V_{Y}\right)+\frac{1}{2} h^{1}\left(V_{Y}\right)+\frac{1}{2} h^{2}\left(V_{Y}\right)\right) \\
& \times \log \left(M_{\mathrm{KK}}^{2} / \mu^{2}\right)+\left.2 \sum_{Y=0, \pm \frac{5}{6}} \operatorname{Tr}_{R_{Y}}\left(Q_{a}^{2}\right) 2 \mathbf{T}\left(S, V_{Y}\right)\right|_{R \rightarrow 1} \\
& +2 \sum_{Y=0, \pm \frac{5}{6}} \operatorname{Tr}_{R_{Y}}\left(Q_{a}^{2}\right)\left[\int_{S}-\frac{1}{2} \operatorname{ch}_{2}\left(V_{Y}\right)-\frac{1}{6} c_{1}\left(V_{Y}\right) c_{1}(T)\right. \\
& \left.-\frac{1}{24} c_{2}(T)\right] \log \left(R^{2} \Lambda^{2}\right),
\end{aligned}
$$

with $M_{\mathrm{KK}}=1 / R_{S}$. Here we have collected the finite pieces in the first two lines and the divergent pieces in the third line. The first line describes the running of the massless modes up to the KK scale. The second line describes the effect of integrating out the KK modes at the KK scale; this term depends only on complex structure moduli. Finally the third line describes the running above the KK scale. We see that the divergent piece is entirely proportional to a local density on $S$, as required by the Lorentz invariance of the underlying eight-dimensional $S U(5)$ gauge theory.

The important thing to notice about (4.37) is that the divergent contributions in the third line are not necessarily proportional to $k_{a}$, and hence cannot necessarily be absorbed in the bare gauge coupling $g$. Indeed, for the case of interest with GUT breaking by hypercharge flux, for $Y=0$ we must take $V_{Y} \rightarrow \mathcal{O}$ where $\mathcal{O}$ is the trivial line bundle on $S$, and for $Y=5 / 6$ we must take $V_{Y} \rightarrow \zeta=L^{5 / 6}$ which satisfies $c_{1}(\zeta) \cdot c_{1}(T)=0$ and $c_{1}(\zeta)^{2}=-2$. Then clearly we find a divergence which is not proportional to $k_{a} \log \left(\Lambda^{2}\right)$.

Of course we already predicted such a divergence earlier. In the 8d YangMills theory with 16 supersymmetries, the $\operatorname{Tr}\left(F^{2}\right)$ terms are not corrected at one loop, but as we discussed there is a one-loop correction of the form $\log \left(\Lambda^{2}\right) t_{8} \operatorname{Tr}_{\text {adj }}\left(F^{4}\right)$. After compactifying on a background with GUT breaking flux, this gives a divergent contribution to the four-dimensional kinetic terms of the form

$$
\log \Lambda^{2} n_{a} \operatorname{Tr} F_{a}^{2}
$$

Note in particular that for the $U(1)_{Y}$ gauge field the $\operatorname{Tr}\left(F^{2}\right) \operatorname{Tr}\left(F^{2}\right)$ piece gives a contribution where the two four-dimensional gauge fields come from different traces, whereas such contributions for the non-abelian gauge fields are always zero. Now one may check using our earlier expressions that 
$\operatorname{Tr}_{\mathbf{R}_{0}}\left(Q_{a}^{2}\right)$ (or equivalently $\operatorname{Tr}_{\mathbf{R}_{5 / 6}}\left(Q_{a}^{2}\right)$ ) is in fact a linear combination of $k_{a}$ and $n_{a}$. Thus, the divergences can be understood as one-loop contributions to the local $\operatorname{Tr}\left(F^{2}\right) \operatorname{Tr}\left(R^{2}\right)$ and $\operatorname{Tr}_{\text {adj }}\left(F^{4}\right)$ terms in eight dimensions, which is precisely what we find in the third line of (4.37).

There are additional divergent contributions coming from the modes living on the matter curves. Indeed anomaly cancellation associated to the $\operatorname{Tr}\left(F^{4}\right)$ terms gives the relation

$$
\left[\Sigma_{\mathbf{5}}\right]-3\left[\Sigma_{\mathbf{1 0}}\right]-5 c_{1}(T)=0
$$

so it would in fact be inconsistent not to include these additional modes. However, we will analyze the contributions to these modes later and see that they cannot cancel the non- $S U(5)$ symmetric divergences in (4.37) proportional to $\operatorname{ch}_{2}\left(V_{Y}\right)$.

Thus the divergence is unavoidable in the local model, and we have to ask how it is naturally cut off in an $F$-theory compactification. Naively one may have thought that the running is naturally cut off when we include modes in the local geometry with masses of order the ten-dimensional Planck scale, which we denote $m_{10}$. However the $\log \Lambda^{2}$ dependence of the oneloop amplitude in the eight-dimensional gauge theory has a simple physical interpretation: it indicates that the back-reaction grows far from the 7-brane. In fact in a IIb weak coupling limit it can be interpreted as the Green's function of closed string field like the RR axion, i.e., the Green's function of the Laplacian in the two dimensions transverse to the 7-brane. Thus, this divergence generally is not cut off by Planck scale modes in the local geometry. In fact as we already mentioned, due to the BPS properties of the $\operatorname{Tr}\left(F^{4}\right)$ term, such massive modes can not contribute at all at one loop $[52,87]$, and the running is unmodified at $m_{10}$.

Instead the divergence is canceled by including suitable 7-branes with opposite charges at infinity, i.e., it corresponds to a 7-brane tadpole cancellation condition in a compact model. This introduces new open string soliton sectors whose ground states are BPS, and so can contribute to the amplitude. If the tadpole is canceled globally (i.e., only for finite $M_{P l}$ ), a natural cut-off would generally be the mass of an open string soliton stretching across $B_{3}$, which is of order $\Lambda \sim m_{10}^{2} V_{B_{3}}^{1 / 6}$. However if it is canceled in a higher order neighborhood of the GUT brane, as in [28], then it could be much smaller. Hence we introduce a fudge parameter $\lambda$, which is model dependent, and write our cut-off as

$$
\Lambda=\lambda \cdot M_{\mathrm{KK}}
$$


where

$$
m_{10} / M_{\mathrm{KK}}<\lambda \leq R_{B_{3}} m_{10}^{2} / M_{\mathrm{KK}}
$$

Here $M_{\mathrm{KK}}=1 / R_{S_{2}}$ is the scale where the KK modes of the eight-dimensional gauge theory become important. ${ }^{9}$

After some rearranging, we can express the running incorporating only the contributions from the $S U(5)$ gauge fields as

$$
\frac{16 \pi^{2}}{g_{a}^{2}(\mu)}=\frac{16 \pi^{2} k_{a}}{\tilde{g}^{2}}+b_{a}^{g} \log \left(\lambda^{2 / 3} M_{\mathrm{KK}}^{2} / \mu^{2}\right)+\delta_{a}^{g},
$$

where

$$
\delta_{a}^{g}=\left.2 b_{a}^{5 / 6}\left(\mathbf{T}_{5 / 6}-\mathbf{T}_{0}\right)\right|_{R \rightarrow 1}
$$

and we used our results on the scaling dependence of the holomorphic torsion to put the $R$ and $\Lambda$ dependence in the second term on the right hand side, and we absorbed several universal pieces by shifting $g \rightarrow \tilde{g}$. We have $b_{a}^{5 / 6}=$ $(50 / 3,6,4)$, and as before $M_{\mathrm{KK}}=1 / R_{S}=V_{S_{2}}^{-1 / 4}$. The logic behind writing the formula this way is that formally if we let $L^{5 / 6} \rightarrow \mathcal{O}$, then $\mathbf{T}_{5 / 6} \rightarrow \mathbf{T}_{\mathcal{O}}$ plus a contribution for the extra zero modes in the representation $\mathbf{R}_{5 / 6}$.

Assuming generic values for the complex structure moduli, the correction $\delta_{a}^{(g)}$ can only give a small numerical correction to the relation between the GUT scale and the KK scale. However, $\lambda$ may depend on the ratio $R_{S_{2}} R_{B_{3}} / l_{10}^{2}$ and give a parametrically large correction, with the relation

$$
M_{\mathrm{GUT}} \sim \lambda^{1 / 3} M_{\mathrm{KK}}
$$

Since we regard the GUT scale as fixed by measurements of couplings at low energies, this means that the KK scale may end up parametrically lower than $10^{16} \mathrm{GeV}$, although we will see that in practice the ratio is presumably not very large.

\footnotetext{
${ }^{9}$ In v1 of this paper, we took the cut-off $\Lambda$ at the lower end. It was emphasized in [55] that $\Lambda$ could be much larger, although the divergence in [55] is due to a $D 5$-tadpole and thus different from the divergence encountered here. We thank J. Conlon for conversations about this issue.
} 


\subsubsection{KK modes of matter fields}

Similarly we may write expressions for the KK towers of the modes localized on the matter curves, $\Sigma_{\overline{\mathbf{5}}}$ and $\Sigma_{\mathbf{1 0}}$. Actually the matter curve $\Sigma_{\overline{\mathbf{5}}}$ is usually singular and it turns out that charged matter naturally propagates on the normalization of the matter curve [17], so in the following we shall actually use the notation $\Sigma_{\overline{5}}$ to denote this normalization. We decompose the $S U(5)$ multiplets as

$$
\begin{aligned}
10 & =(2,3)_{1 / 6}+(1,1)_{1}+(1, \overline{3})_{-2 / 3} \\
\overline{5} & =(2,1)_{-1 / 2}+(1, \overline{3})_{1 / 3}
\end{aligned}
$$

Again it is a convenient shorthand to omit the $S U(3) \times S U(2)$ transformation properties and denote these representations by their hypercharge, $\mathbf{R}_{Y}$. Let us concentrate on $\Sigma_{\mathbf{1 0}}$. The total flux on this curve consists of the flux of a line bundle $M_{\mathbf{1 0}}$ which we may think of as coming from a non-compact 7-brane, and the flux of the hypercharge gauge field (corresponding to a line bundle $\left.L^{Y}\right)$. We consider the Laplacian $\Delta_{n, Y}=\left(\bar{\partial}+\bar{\partial}^{\dagger}\right)^{2}$ acting on

$$
\left.\left.\Omega_{\Sigma}^{0, n} \otimes M_{10}\right|_{\Sigma} \otimes L^{Y}\right|_{\Sigma} \otimes K_{\Sigma}^{1 / 2} .
$$

There should be no confusion with the Laplacians $\Delta_{n, Y}$ on $S$ introduced earlier, because they act on representations with different values of $Y$. For each eigenvector of $\Delta_{n, Y}$ we get a chiral or anti-chiral field (depending on whether $n=0$ or $n=1$ ) in the representation $\mathbf{R}_{Y}$. Thus we will need the following combination of determinants:

$$
\mathbf{K}_{\Sigma_{\mathbf{1 0}}, Y}=-\frac{1}{2} \log \operatorname{det}^{\prime} \frac{\Delta_{0, Y}}{\Lambda^{2}}-\frac{1}{2} \log \operatorname{det}^{\prime} \frac{\Delta_{1, Y}}{\Lambda^{2}} .
$$

In fact, it is not hard to see that the massive spectra of $\Delta_{0, Y}$ and $\Delta_{1, Y}$ are identical. Intuitively, this is the statement that we need a chiral and an anti-chiral fermion in order to write down a mass term. Hence modulo the $\Lambda$ dependence we are actually interested in twice the holomorphic Ray-Singer torsion on $\Sigma_{10}$ :

$$
\mathbf{K}_{\Sigma_{\mathbf{1 0}, Y}}=-2 \mathbf{T}_{M_{\mathbf{1 0}} \otimes L^{Y} \otimes K_{\Sigma}^{1 / 2}}+\zeta(0) \log \Lambda^{2} .
$$

The resulting contributions to the threshold corrections are summarized in table 2. These expressions really apply to the total matter curves, so it includes possible matter curves where the Higgses or additional messenger fields for supersymmetry (SUSY) breaking are localized, if these are different from the curves where quarks and lepton are localized. 
Table 2: KK Threshold corrections.

\begin{tabular}{llll}
\hline & $S_{a}^{g}$ & $S_{a}^{\mathbf{1 0}}$ & $S_{a}^{\overline{\mathbf{5}}}$ \\
\hline$U(1)_{Y}$ & $\frac{50}{3} \mathbf{K}_{5 / 6}$ & $\frac{1}{3} \mathbf{K}_{\Sigma_{\mathbf{1 0}}, 1 / 6}+2 \mathbf{K}_{\Sigma_{\mathbf{1 0}}, 1}$ & $\mathbf{K}_{\Sigma_{\overline{5}},-1 / 2}+\frac{2}{3} \mathbf{K}_{\Sigma_{\overline{\mathbf{5}}}, 1 / 3}$ \\
& & $+\frac{8}{3} \mathbf{K}_{\Sigma_{\mathbf{1 0}},-2 / 3}$ & \\
$S U(2)$ & $4 \mathbf{K}_{0}+6 \mathbf{K}_{5 / 6}$ & $3 \mathbf{K}_{\Sigma_{\mathbf{1 0}}, 1 / 6}$ & $\mathbf{K}_{\Sigma_{\overline{5}},-1 / 2}$ \\
$S U(3)$ & $6 \mathbf{K}_{0}+4 \mathbf{K}_{5 / 6}$ & $2 \mathbf{K}_{\Sigma_{\mathbf{1 0}}, 1 / 6}+\mathbf{K}_{\Sigma_{\mathbf{1 0}},-2 / 3}$ & $\mathbf{K}_{\Sigma_{\overline{\mathbf{5}}}, 1 / 3}$ \\
\hline
\end{tabular}

Again it is convenient to separate out the divergent pieces. Using the result from Appendix B, we have

$$
K_{\Sigma, Y}=-2 \mathbf{T}(\Sigma, V)_{R \rightarrow 1}-\left[h^{0}(V)-\int_{\Sigma} \frac{1}{2} c_{1}(V)+\frac{1}{6} c_{1}(T)\right] \log \left(R^{2} \Lambda^{2}\right) .
$$

Further we may rewrite $h^{0}=\frac{1}{2} \chi(V)+\frac{1}{2} h^{0}+\frac{1}{2} h^{1}$ and use Riemann-Roch:

$$
\chi(V)=\int_{\Sigma} c_{1}(V)+\frac{1}{2} c_{1}(T)
$$

Then we can write the contribution of the massless and massive modes on $\Sigma$ to the running as

$$
\begin{aligned}
\frac{16 \pi^{2}}{g_{a}^{2}(\mu)}= & \frac{16 \pi^{2} k_{a}}{g^{2}}+\sum_{Y} 2 \operatorname{Tr}_{R_{Y}}\left(Q_{a}^{2}\right)\left(\frac{1}{2} h^{0}\left(V_{Y}\right)+\frac{1}{2} h^{1}\left(V_{Y}\right)\right) \log \left(M_{\mathrm{KK}}^{2} / \mu^{2}\right) \\
& -\left.\sum_{Y} 2 \operatorname{Tr}_{R_{Y}}\left(Q_{a}^{2}\right) 2 \mathbf{T}\left(\Sigma, V_{Y}\right)\right|_{R \rightarrow 1} \\
& -\sum_{Y} 2 \operatorname{Tr}_{R_{Y}}\left(Q_{a}^{2}\right) \frac{1}{12} \chi(\Sigma) \log \left(R_{\Sigma}^{2} \Lambda^{2}\right)
\end{aligned}
$$

where we used $\int_{\Sigma} c_{1}(T)=\chi(\Sigma)$. Also $M_{\mathrm{KK}}$ is strictly given by $1 / R_{\Sigma}$, but normally we would expect that $R_{\Sigma} \sim R_{S}$. From the third line we see that the divergent terms are $S U(5)$ symmetric and can be absorbed in the bare coupling, as we promised earlier.

Putting all the one-loop KK corrections together, we get

$$
\frac{16 \pi^{2}}{g_{a}^{2}(\mu)}=\frac{16 \pi^{2} k_{a}}{g^{2}}+b_{a} \log \left(\Lambda^{2} / \mu^{2}\right)+S_{a}^{g}+S_{a}^{\mathbf{1 0}}+S_{a}^{\overline{\mathbf{5}}}
$$


where $S_{a}^{g}, S_{a}^{\mathbf{1 0}}$ and $S_{a}^{\overline{\mathbf{5}}}$ are given in table 2. According to our earlier results, we may write this as

$$
\begin{aligned}
\frac{16 \pi^{2}}{g_{a}^{2}(\mu)}= & \frac{16 \pi^{2} k_{a}}{\tilde{g}^{2}}+\left(b_{a}^{(g)}+b_{a}^{(c)}+b_{a}^{(h)}\right) \log \frac{M_{\mathrm{KK}}^{2}}{\mu^{2}}+\delta_{a}^{g}+\delta_{a}^{10}+\delta_{a}^{\overline{5}} \\
& -2 \operatorname{Tr}_{R_{5 / 6}}\left(Q_{a}^{2}\right) \int_{S} \operatorname{ch}_{2}\left(L^{5 / 6}\right) \log \left(\Lambda^{2} / M_{\mathrm{KK}}^{2}\right) .
\end{aligned}
$$

By $\delta_{a}^{\mathbf{1 0}}, \delta_{a}^{\overline{5}}$ we mean the the same expressions as in table 2, except with the scale dependent $\log \Lambda^{2} R_{\mathrm{KK}}^{2}$ terms removed. (In other words, we take $\mathbf{K}_{\Sigma, Y}=-2 \mathbf{T}_{\Sigma, Y}$ with the volume of the matter curves set to one, $\left.R_{\mathrm{KK}}=1\right)$. Also $b_{a}=b_{a}^{g}+b_{a}^{(c)}+b_{a}^{(h)}=(11,1,-3)$ are the beta function coefficients of the MSSM, and

$$
\int_{S} \operatorname{ch}_{2}\left(L^{5 / 6}\right)=\frac{1}{2} c_{1}\left(L^{5 / 6}\right)^{2}=-1
$$

We would like to further rewrite the formula so that we can compare it to running in a conventional four-dimensional model. This can be done by absorbing various further pieces in the bare coupling. Note that

$$
2 \operatorname{Tr}_{R_{5 / 6}}\left(Q_{a}^{2}\right) \log \left(\Lambda^{2} / M_{\mathrm{KK}}^{2}\right)=-\frac{3}{2} 2 \operatorname{Tr}_{R_{0}}\left(Q_{a}^{2}\right) \log \left(\lambda^{2 / 3}\right) \bmod k_{a} .
$$

The chiral fields come in complete $S U(5)$ multiplets, so

$$
b_{a}^{(c)} \log \left(\lambda^{2 / 3}\right)=0 \bmod k_{a} .
$$

The higgses do not come in a complete multiplet, but we may write

$$
b_{a}^{(h)} \log \left(\lambda^{2 / 3}\right)+b_{a}^{(T)} \log \left(\lambda^{2 / 3}\right)=0 \bmod k_{a},
$$

where $T$ stands for the triplet $S U(5)$ partners of the Higgses. Then by absorbing various $S U(5)$ symmetric pieces in the bare coupling, we can rewrite the formula as

$$
\frac{16 \pi^{2}}{g_{a}^{2}(\mu)}=\frac{16 \pi^{2} k_{a}}{\tilde{g}^{2}}+b_{a}^{M S S M} \log \frac{M_{\mathrm{GUT}}^{2}}{\mu^{2}}+b_{a}^{(T)} \log \frac{M_{\mathrm{GUT}}^{2}}{M_{\mathrm{KK}}^{2}}+\delta_{a}^{g}+\delta_{a}^{\mathbf{1 0}}+\delta_{a}^{\overline{5}},
$$

where

$$
M_{\mathrm{GUT}}=\lambda^{1 / 3} M_{\mathrm{KK}}
$$


and $b_{a}^{(T)}=(2 / 3,0,1)$. In other words, comparing with the analogous formula for conventional four-dimensional models, it looks like unification gets modified by the appearance of an effective pair of Higgs triplets with masses $M_{T}^{\mathrm{eff}}=M_{\mathrm{KK}}$ below the GUT scale, as well as some small corrections $\delta_{a}$ from the GUT breaking sector.

One may contrast the situation in $F$-theory models with earlier work on compactifications of $M$-theory on ALE-fibered manifolds of $G_{2}$ holonomy [18]. In that case charged matter is localized at points on the $G_{2}$ manifold and so does not contribute a tower of KK modes to the one-loop running, and there was no parametric separation between the KK scale and the GUT scale. The situation in $F$-theory is a bit more complicated.

Recently the moduli dependence of the effective couplings has been considered from the point of view of $4 d$ supergravity, where the NSVZ-KL formula relates holomorphic couplings to physical couplings in the effective four-dimensional supergravity theory $[53,54]$ :

$$
\frac{1}{g_{a}^{2}(\mu)}=\operatorname{Re}\left(f_{a}\right)+b_{a} \log \left(\frac{\Lambda}{\mu}\right)+\log g_{a}^{2}-c_{a} \hat{\mathcal{K}}-\log \operatorname{det} Z
$$

In particular, it was argued that this formula predicts a new unification scale [55]. Namely after substituting $\Lambda \sim M_{P l}, f_{a} \sim f, \hat{\mathcal{K}}=-2 \log \mathcal{V}$ and $Z \sim \mathcal{V}^{-2 / 3}$, we can recombine the terms and find an effective shift $M_{P l} \rightarrow$ $M_{P l} \mathcal{V}^{-1 / 3}$. Furthermore, this shift was argued to be due to tadpole cancellation from a microscopic point of view, i.e., the appearance of new states at this scale which make some divergent loop integrals finite.

We find the claims of [55] somewhat surprising because the effective $4 d$ supergravity does not know about such microscopic details of the compactification. To illustrate this, suppose we broke the GUT group through discrete Wilson lines instead of fluxes. Some toy models of this type were constructed in [15] (they have some exotics, but that is besides the point here). The divergent terms only depend on the $F_{Y}$ field strength, so in the case of discrete Wilson line breaking all divergent terms must be $S U(5)$ symmetric and cannot affect the unification scale. Thus in this case the unification scale must be the $\mathrm{KK}$ scale $M_{\mathrm{KK}}=1 / R_{S}$, as we also find from the formulae derived above. Yet the argument in [55] is insensitive to such microscopic details. Also the idea that tadpoles can only be canceled when $M_{P l}$ is finite is not quite true, see the discussion in [28]. It can already happen on the second order neighborhood of the brane. We believe this indicates that the appearance of the new "winding" scale purely from a generic supergravity argument is likely not physical. 


\subsection{Toy models}

We would like to be able to get an idea of the size of the heavy threshold corrections, and their effect on unification. Before we start, we state some general formulae. With some simple algebra, the relation between the GUT scale parameters and the parameters measured at low energies can be expressed as [56]

$$
\begin{aligned}
\sin ^{2} \theta_{W}\left(M_{Z}\right) & =\frac{3}{8}\left[1-\left(b_{1}-\frac{5}{3} b_{2}\right) \frac{a}{4 \pi} \log \left(M_{\mathrm{GUT}}^{2} / M_{Z}^{2}\right)\right]+\delta^{\sin } \\
\alpha_{3}^{-1}\left(M_{Z}\right) & =\frac{3}{8}\left[\frac{1}{a}-\left(b_{1}+b_{2}-\frac{8}{3} b_{3}\right) \frac{1}{4 \pi} \log \left(M_{\mathrm{GUT}}^{2} / M_{Z}^{2}\right)\right]+\delta^{\alpha_{3}},
\end{aligned}
$$

where

$$
\begin{aligned}
\delta^{\sin } & =-\frac{3}{8} \frac{a}{4 \pi}\left(\delta_{1}-\frac{5}{3} \delta_{2}\right) \\
\delta^{\alpha_{3}} & =-\frac{3}{8} \frac{1}{4 \pi}\left(\delta_{1}+\delta_{2}-\frac{8}{3} \delta_{3}\right)
\end{aligned}
$$

and $a^{-1}=\alpha_{\mathrm{em}}^{-1}\left(M_{Z}\right)=127.9 \pm 0.1$.

These equations will be used in the following way. We use the experimental values of $\alpha_{\mathrm{em}}\left(M_{Z}\right)$ and $\sin ^{2} \theta_{W}\left(M_{Z}\right)$ to predict $M_{\mathrm{GUT}}$. Then we run them back down to predict $\alpha_{3}\left(M_{Z}\right)$. Further the apparent GUT scale $\bar{M}_{\mathrm{GUT}}$, defined as the scale where $\alpha_{1}^{-1}$ and $\alpha_{2}^{-1}$ meet by extrapolating from low energy data, is given by

$$
\bar{M}_{\mathrm{GUT}}^{2}=M_{\mathrm{GUT}}^{2} \exp \frac{\delta_{1}-\frac{5}{3} \delta_{2}}{b_{1}-\frac{5}{3} b_{2}}=M_{\mathrm{KK}}^{2} \lambda^{2 / 3} \exp \frac{\delta_{1}-\frac{5}{3} \delta_{2}}{b_{1}-\frac{5}{3} b_{2}} .
$$

The heavy threshold corrections affect the relation between the value of the KK scale and the apparent GUT scale, but not the value of the apparent GUT scale because this is defined by extrapolating low energy data.

The experimental values in the $\overline{\mathrm{MS}}$ scheme are given by

$$
\sin ^{2} \theta_{W}\left(M_{Z}\right)=0.2312 \pm 0.0002, \quad \alpha_{3}\left(M_{Z}\right)=0.1176 \pm 0.002
$$

Then without stringy threshold corrections, $M_{Z} \sim 91.2 \mathrm{GeV}$, and using $\alpha_{\mathrm{em}}^{-1}\left(M_{Z}\right)$ and $\sin ^{2} \theta_{W}\left(M_{Z}\right)$ as input, we find

$$
M_{\mathrm{GUT}}=1.8 \times 10^{16} \mathrm{GeV}, \quad \alpha_{\mathrm{GUT}}^{-1} \sim 24.4, \quad \alpha_{3}\left(M_{Z}\right)=0.115
$$


We see that although $\alpha_{3}^{-1}$ comes out slightly too large, the one loop running of the MSSM gives a remarkably accurate prediction.

However we should really include various corrections due to two loop running, scheme conversion, and light SUSY thresholds. The effects can be summarized [57-59] by adding a correction $\delta_{3}^{2-\text { loop }} / 4 \pi \sim-0.82$ and $\delta_{3}^{\text {light }} / 4 \pi \sim$ -0.12 . Including these effects, instead we find $\alpha_{3}\left(M_{Z}\right) \sim 0.129$ which is a much larger discrepancy. Turning it around and using the experimental value of $\alpha_{3}\left(M_{Z}\right)$ as input, we would get $\alpha_{3}\left(\bar{M}_{\mathrm{GUT}}\right)=\alpha_{\mathrm{GUT}}\left(1+\epsilon_{3}\right)$ where $\epsilon_{3} \sim-4 \%$. In order to fix this, from equation (4.62) we see that we effectively need a positive contribution of the heavy thresholds of the order $\delta^{\alpha_{3}} \sim+0.94$.

Our result for the leading corrections is given in equation (4.53) or equivalently equation (4.58). We see that among these, there is one universal contribution that we have written as the contribution of an effective pair of triplets $b_{a}^{(T)} \log M_{\mathrm{GUT}}^{2} / M_{\mathrm{KK}}^{2}$. Now it is well-known in the context of fourdimensional $S U(5)$ models that lowering the masses of the Higgs triplets improves agreement with measured deviations from unification, so this looks like good news. However in four-dimensional $S U(5)$ models, this is also one of the main sources of tension, as lowering the triplet masses also enhances dimension five proton decay $[60,61]$. In our KK models we have an analogous issue because the intermediate states are tied to the KK scale, and dimension five proton decay operators need to be sufficiently suppressed. Moreover we cannot choose the KK scale freely as it is tied to the ratio of the GUT and Planck scales.

Let us illustrate this with some numerics. Suppose that $M_{\mathrm{GUT}} / M_{\mathrm{KK}} \sim$ $10^{2}$. Using the above formulae, and including the two-loop and light SUSY threshold corrections, we find that the unification scale gets lowered to $1.3 \times 10^{16} \mathrm{GeV}$ and the prediction for $\alpha_{3}\left(M_{Z}\right)$ is 0.115 , so we have essentially restored the successful prediction. If on the other hand we have $M_{\mathrm{GUT}} / M_{\mathrm{KK}} \sim 10^{0.5}$, then the unification scale is $1.7 \times 10^{16} \mathrm{GeV}$ and the predicted value for $\alpha_{3}\left(M_{Z}\right)$ is 0.126 . In this case, the remaining heavy threshold corrections have to make up for the difference.

In the remainder we would like to discuss the model dependent threshold corrections. We will compute the determinants for some toy models, the special cases of line bundles $\mathcal{O}(n,-n)$ on $\mathbf{P}^{1} \times \mathbf{P}^{1}$ and line bundles on genus one matter curves.

In order to compute the torsion for $\mathcal{O}(n,-n)$, we first need the torsion for the line bundle $\mathcal{O}(k)_{\mathbf{P}^{1}}$. The eigenvalues we need are well-known - they are just the energy eigenvalues for a charged particle moving on a sphere in 
a magnetic field. For the sphere, the eigenvalues of the Laplacian on zero forms are given by $l(l+1)$ with multiplicity $2 l+1$. The eigenvalues of $\Delta_{k}$ are given by $l(l+|k+1|)$ with multiplicity $2 l+|k+1|$. Thus we need

$$
\zeta_{k}(s)=\sum_{l} \frac{2 l+|k+1|}{l^{s}(l+|k+1|)^{s}} .
$$

This was evaluated in [62-64], with the result

$$
\zeta_{k}^{\prime}(0)=4 \zeta_{R}^{\prime}(-1)-\frac{1}{2}(k+1)^{2}+\sum_{m=1}^{k+1}(2 m-|k+1|) \log m,
$$

where $\zeta_{R}^{\prime}(-1) \sim-0.165421$. Note that this is symmetric under $k \rightarrow-2-k$. The torsion is then given by $\left.\mathbf{T}_{\mathcal{O}(k)}\right|_{R \rightarrow 1}=-\frac{1}{2} \zeta_{k}^{\prime}(0)$.

Now we can use this to compute $\mathbf{K}_{Y}$. We use the product formula for Ray-Singer torsion $[48,65]$

$$
\mathbf{T}\left(M_{1} \times M_{2}, E_{1} \times E_{2}\right)=\chi\left(M_{1}, E_{1}\right) \mathbf{T}\left(M_{2}, E_{2}\right)+\mathbf{T}\left(M_{1}, E_{1}\right) \chi\left(M_{2}, E_{2}\right) .
$$

Since $\chi\left(\mathbf{P}^{1}, \mathcal{O}(n)\right)=n+1$ we have

$$
\mathbf{T}_{\mathcal{O}(n, m)}=(n+1) \mathbf{T}_{\mathcal{O}(m)}+(m+1) \mathbf{T}_{\mathcal{O}(n)} .
$$

We take the radii of the two $\mathbf{P}^{1}$ 's to be equal, since we imagine an embedding of $\mathbf{P}^{1} \times \mathbf{P}^{1}$ in $B_{3}$ such that the difference between the two rulings becomes homologically trivial. Now we can take $L^{5 / 6}=\mathcal{O}(n,-n), n \neq 0$, so that

$$
\begin{aligned}
\delta_{a}^{g} & =\left.2 b_{a}^{5 / 6}\left(\mathbf{T}_{5 / 6}-\mathbf{T}_{0}\right)\right|_{R=1} \\
& =\left.2 b_{a}^{5 / 6}\left((n+1) \mathbf{T}_{\mathcal{O}(-n)}+(1-n) \mathbf{T}_{\mathcal{O}(n)}-2 \mathbf{T}_{\mathcal{O}(0)}\right)\right|_{R=1}
\end{aligned}
$$

with $b_{a}^{5 / 6}=(50 / 3,6,4)$. In order to have no massless triplet gauge bosons we need $n=1$, but the model makes sense for any $n$. Using (4.62), for $n=1$ we get

$$
\begin{aligned}
\delta^{g, \sin } & =-\left.\frac{5 a}{4 \pi}\left(2 \mathbf{T}_{\mathcal{O}(-1)}-2 \mathbf{T}_{\mathcal{O}(0)}\right)\right|_{R=1}=\frac{5 a}{8 \pi} \\
\delta^{g, \alpha_{3}} & =-\left.\frac{9}{4 \pi}\left(2 \mathbf{T}_{\mathcal{O}(-1)}-2 \mathbf{T}_{\mathcal{O}(0)}\right)\right|_{R=1}=\frac{9}{8 \pi}
\end{aligned}
$$


Thus we see in this toy example that the corrections work in opposite directions: for $n=1$ the sign of $\delta^{g, \sin }$ is positive so the KK scale is increased, decreasing $\alpha_{3}^{-1}\left(M_{Z}\right)$, but the sign of sign of $\delta^{g, \alpha_{3}}$ serves to reduce the discrepancy. Plugging in mathematica, the net effect however is a slight increase in the discrepancy.

Now let us examine contributions from the matter curves. We discuss first $\Sigma_{\mathbf{1 0}}$ but the case of $\Sigma_{\overline{\mathbf{5}}}$ is completely analogous. Since the net GUT breaking flux through each matter curve must be zero, the matter curve cannot be a $\mathbf{P}^{1}$. Thus the first non-trivial case corresponds to $\Sigma_{\mathbf{1 0}}$ having genus one. Let us recall some results about Ray-Singer torsion for $T^{2}$, which we take to be flat with modular parameter $\tau$. For flat line bundles we have [48]

$$
\mathbf{T}_{L_{z}}=\log \left|\mathrm{e}^{\pi \mathrm{i} v^{2} \tau} \vartheta_{1}(z, \tau) \eta(\tau)^{-1}\right|,
$$

where $z=u-\tau v$ specifies a point on the dual $T^{2}$, and $L_{z}$ is the corresponding line bundle on $T^{2}$. When $z \rightarrow 0$, the theta function has a zero and the torsion is minus infinity. This is because one of the eigenvalues of the Laplacian goes to zero in this limit. Thus for $z=0$ (i.e., for the trivial line bundle), we have to remove this zero and the torsion is instead given by

$$
\mathbf{T}_{\mathcal{O}}=\lim _{z \rightarrow 0} \mathbf{T}_{L_{z}}-\frac{1}{2} \log \lambda_{0}=\lim _{z \rightarrow 0} \mathbf{T}_{L_{z}}-\frac{1}{2} \log \frac{4 \pi^{2}|z|^{2}}{\operatorname{Im}(\tau)^{2}} .
$$

Now we consider

$$
\left.L^{1 / 6}\right|_{\Sigma_{\mathbf{1 0}}} \otimes M_{\mathbf{1 0}} \otimes K_{\Sigma_{\mathbf{1 0}}}^{1 / 2}=\mathcal{O},\left.\quad L^{5 / 6}\right|_{\Sigma_{\mathbf{1 0}}}=L_{z} .
$$

Using (4.62), this leads to the following corrections:

$$
\begin{aligned}
\delta^{10, \text { sin }} & =-\frac{3}{8} \frac{a_{\mathrm{em}}}{4 \pi}\left(\frac{28}{3} \mathbf{T}_{\mathcal{O}}-\frac{28}{3} \mathbf{T}_{L_{z}}\right), \\
\delta^{10, \alpha_{3}} & =-\frac{3}{8} \frac{1}{4 \pi}\left(4 \mathbf{T}_{\mathcal{O}}-4 \mathbf{T}_{L_{z}}\right),
\end{aligned}
$$

where we used that $\mathbf{T}_{L_{z}}=\mathbf{T}_{L_{-z}}$ and all the torsions should be evaluated at $R \rightarrow 1$. Evaluating in mathematica for generic $\tau$ and $z$, the corrections work in opposite directions, and the net effect is again a slight increase in the discrepancy. For generic values of the moduli, the eigenvalues are of order the compactification scale and the corrections they give upon integrating out are small. For special values of the moduli, in this example $z \rightarrow 0$, an eigenvalue may become parametrically lighter than the KK scale and give large corrections. In our example we can easily find small values of $z$ which repair the discrepancy, e.g., for $\tau=I$ and $z=0.1-0.1 I$ (and including the 

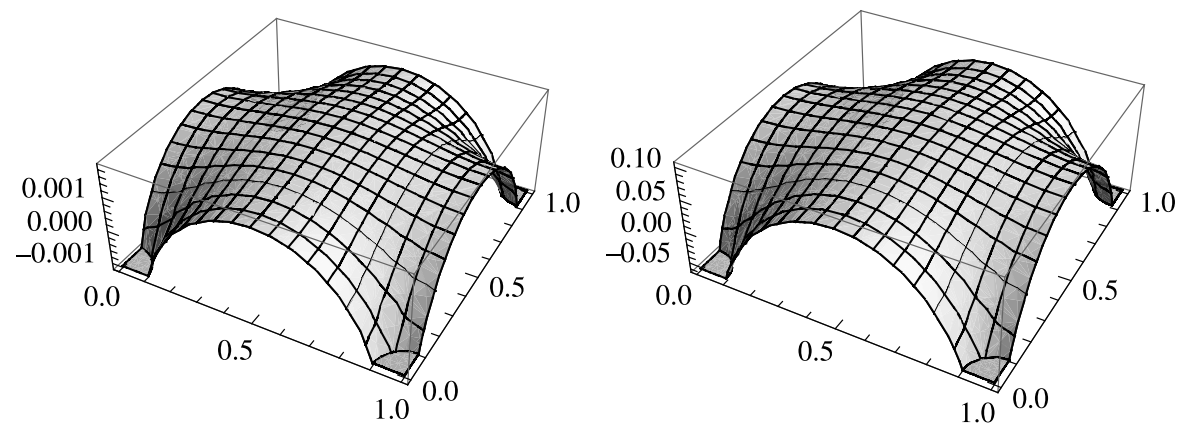

Figure 2: A plot of the threshold corrections (4.75) localized on $\Sigma_{\mathbf{1 0}}$, here assumed to be a square torus. On the left the value of $\delta^{\mathbf{1 0}}$,sin is indicated along the $z$-axis, on the right the $z$-axis measures the value of $\delta^{\mathbf{1 0}, \alpha_{3}}$. The remaining axes correspond to the bundle modulus $z=x-\tau y$ with $0 \leq x, y \leq 1$.

two-loop and light SUSY thresholds, but none of the other heavy thresholds) we find $\alpha_{3}\left(M_{Z}\right) \sim 0.1157$. However this correction then comes mainly from parametrically light charged states which presumably enhance proton decay significantly.

Similarly we can consider a toy model for the threshold corrections coming from $\Sigma_{\overline{\mathbf{5}}}$. Typically this matter curve has genus larger than that of $\Sigma_{\mathbf{1 0}}$, however just to get an idea for the size of the corrections let us assume that $\Sigma_{\overline{5}}$ has genus one and

$$
\left.L^{-1 / 2}\right|_{\Sigma_{\overline{\mathbf{5}}}} \otimes M_{\overline{\mathbf{5}}} \otimes K_{\Sigma_{\overline{\mathbf{5}}}}^{1 / 2}=\left.\mathcal{O} \quad L^{5 / 6}\right|_{\Sigma_{\overline{\mathbf{5}}}}=L_{z}
$$

Then we find

$$
\begin{aligned}
\delta^{\overline{\mathbf{5}}, \sin } & =-\frac{3}{8} \frac{a_{\mathrm{em}}}{4 \pi}\left(\frac{4}{3} \mathbf{T}_{\mathcal{O}}-\frac{4}{3} \mathbf{T}_{L_{z}}\right), \\
\delta^{\overline{\mathbf{5}}, \alpha_{3}} & =-\frac{3}{8} \frac{1}{4 \pi}\left(-4 \mathbf{T}_{\mathcal{O}}+4 \mathbf{T}_{L_{z}}\right) .
\end{aligned}
$$

Again, it is dangerous to compare these contributions to those from $\Sigma_{\mathbf{1 0}}$, since in a given model the curves and line bundles on them cannot be identical. At any rate as long as $\mathbf{T}_{\mathcal{O}}-\mathbf{T}_{L_{z}}$ is positive, which according to the results for (4.75) is true for a large range of $z$, the effect of both corrections is to reduce the discrepancy. 


\subsection{Conclusions}

Since this section was rather long, we would here like to summarize our conclusions. We argued that the full expression for the leading corrections to the gauge kinetic terms in a local model is given by

$$
\frac{16 \pi^{2}}{g_{a}^{2}(\mu)}=\frac{16 \pi^{2} k_{a}}{g^{2}}+b_{a} \log \frac{M_{\mathrm{GUT}}^{2}}{\mu^{2}}+b_{a}^{(T)} \log \frac{M_{\mathrm{GUT}}^{2}}{M_{\mathrm{KK}}^{2}}+\delta_{a}^{g}+\delta_{a}^{\mathbf{1 0}}+\delta_{a}^{\overline{\mathbf{5}}}+\delta_{a}^{(\text {other })}
$$

The correction $\delta_{a}^{\text {(other) }}$ is due to two-loop running and light SUSY thresholds. The correction $b_{a}^{(T)} \log M_{\mathrm{GUT}}^{2} / M_{\mathrm{KK}}^{2}$ is suggestively written as a color triplet correction and has the same effect as lowering the Higgs triplet masses in conventional $4 d$ GUTs, although it had a rather different origin as a one-loop divergence in $F$-theory. It improves the agreement between the predicted and the measured value of $\alpha_{3}\left(M_{Z}\right)$, which as we reviewed differs slightly from the naive value expected form unification. However, to get complete agreement requires lowering $M_{\mathrm{KK}}$ by two orders of magnitude. This is hard to obtain even if we assume dimension five proton decay can be sufficiently suppressed, as the ratio $M_{\mathrm{KK}} / M_{\mathrm{GUT}}$ is tied to the Planck scale and cannot be very large. Thus the remaining discrepancy must come from the correction terms $\delta_{a}^{g}, \delta_{a}^{\mathbf{1 0}}$ and $\delta_{a}^{\overline{\mathbf{5}}}$. We showed that they can be expressed in terms of Ray-Singer torsion which depends only on complex structure moduli. In our toy models, these one loop corrections could either increase or decrease $\alpha_{3}\left(M_{Z}\right)$, but if the values obtained in figure 2 are typical, they seem a bit too small to make up the difference (recall we need $\delta_{\text {heavy }}^{\alpha_{3}} \sim+0.94$ ). Hence we conclude that the threshold corrections generally go in the right direction, but precise agreement with experimentally measured deviations from unification probably implies extra structure beyond what is assumed here.

There are some interesting differences with $4 d$ models. We saw that the KK scale is lowered compared to the GUT scale. This could affect some of the phenomenology because masses of KK modes and certain stabilized moduli are set by $M_{\mathrm{KK}}$ or some power thereof, rather than $M_{\mathrm{GUT}}$. Analogous to four-dimensional models with an extra pair of Higgs triplets and $M_{T}<M_{\mathrm{GUT}}$, this lower scale improves the agreement with measured deviations from unification (through the $b_{a}^{(T)} \log M_{\mathrm{GUT}}^{2} / M_{\mathrm{KK}}^{2}$ term) and enhances dimension five proton decay if present. Different from four-dimensional models, the effective scale $M_{T} \sim M_{\mathrm{KK}}$ is tied to the ratio of the GUT and Planck scales, and dimension six proton decay is also set by this scale. This will be discussed in more detail in the next section. 
However in practice this effect is rather small. The two scales differ by a factor

$$
M_{\mathrm{GUT}} / M_{\mathrm{KK}}=\lambda^{1 / 3}
$$

Although $\lambda$ depends on the UV completion, generically we should expect $\lambda=\Lambda / M_{\mathrm{KK}} \sim R_{B_{3}} R_{S} m_{10}^{2}$. At tree level, we have $m_{10} \sim M_{\mathrm{KK}} \alpha_{\mathrm{GUT}}^{-1 / 4}$ and hence

$$
\lambda \sim M_{P l}^{1 / 3} M_{\mathrm{KK}}^{-1 / 3} \alpha_{\mathrm{GUT}}^{-1 / 6} \sim M_{P l}^{1 / 3} M_{\mathrm{KK}}^{-1 / 3}
$$

since $\alpha_{\mathrm{GUT}}^{-1 / 6} \sim 25^{1 / 6} \sim 1$. Then we have the relation

$$
M_{\mathrm{KK}} \sim M_{\mathrm{GUT}}\left(M_{\mathrm{GUT}} / M_{P l}\right)^{1 / 8} .
$$

Here $M_{P l}$ is the reduced Planck mass, $M_{P l} \sim 2.4 \times 10^{18} \mathrm{GeV}$. With $M_{\mathrm{GUT}} /$ $M_{P l} \sim 10^{-2}$, the KK scale ends up less than half an order of magnitude below the GUT scale.

While we are at it, we believe that a conceptually cleaner approach would be to define a notion of Ray-Singer torsion directly for Higgs bundles. That is, we should really consider the Laplacian for $\bar{D}=\bar{\partial}_{A}+\Phi$ and then define the "Higgs bundle torsion" as in equation (4.29), but with the modified Laplacian. Then in (4.78) we would get a single correction $\delta_{a}^{\text {Higgs }}$. This should reduce to $\delta_{a}^{g}+\delta_{a}^{\mathbf{1 0}}+\delta_{a}^{\overline{5}}$, because the modes become sharply localized on the matter curve when the brane intersection angles are large, and this is controlled by a Kähler modulus. Recall that the torsion is essentially independent of Kähler moduli. (See however [72]).

It is not completely clear how we could go about computing such "Higgs bundle torsion" directly. However apart from a divergent term it should be independent of the Kähler moduli, so we can use heterotic/F-theory duality to compute it. On the heterotic side, the threshold corrections are computed by the torsion of the $E_{8}$ bundle [66,67]. Using results of [68], we expect that in a suitable limit we get the local $F$-theory contribution.

One may also apply our techniques to study threshold corrections for perturbative IIb GUT models. Here one has two expansion parameters and so one should distinguish between $\alpha^{\prime}$ and $g_{s}$ corrections. The former were investigated in [69]. The one-loop contribution would also give rise to holomorphic Ray-Singer torsion. 


\section{Proton decay}

In this section we discuss some constraints on $F$-theory models due to observational constraints on proton decay ${ }^{10}$. We will denote the $\overline{\mathbf{5}}$ modes consisting of $\left(L, d^{c}\right)$ by $\overline{\mathbf{5}}_{m}$ ( $m$ for 'matter) and the $\overline{\mathbf{5}}$ mode consisting of $\left(H_{d}, T_{d}\right)$ by $\overline{\mathbf{5}}_{h}$. Of course we should engineer our model so that after breaking the GUT group there are no massless color triplets. Massless chiral matter is assumed to live on 7-brane intersections rather than in the bulk of the 7-brane. Some issues in models with bulk matter have been clarified recently and can now also be discussed $[70,71]$.

\subsection{Fibered root systems and their monodromies}

In order to be fairly concrete, we first recall some aspects of local geometries for 7-branes and the description of the matter curves in terms of spectral covers $[14,75,76]$. Some of the statements here are not quite rigorous. A more rigorous description can be found in [22].

We focus on an $E_{8}$ ALE (or $d P_{8}$ ) fibration over our surface $S_{2}$, unfolded to an $S U(5)$ singularity. The two-cycles of the ALE are labelled by the roots of $E_{8}$, in such a way that the intersection matrix of the simple roots agrees with the Cartan matrix of $E_{8}$. We will use the labelling shown in figure 3 . We pick a maximal subgroup $S U(5)_{H} \times S U(5)_{\text {GUT }}$ where

$$
\left\{\alpha_{-\theta}, \alpha_{1}, \alpha_{2}, \alpha_{3}\right\}
$$

are the creation operators for the adjoint representation of $S U(5)_{H}$, and similarly

$$
\left\{\alpha_{5}, \alpha_{6}, \alpha_{7}, \alpha_{8}\right\}
$$

are the creation operators for the adjoint representation of $S U(5)_{\mathrm{GUT}}$. Here $\alpha_{-\theta}$ is the negative of the highest root, so that

$$
\alpha_{-\theta}=-\sum d_{i} \alpha_{i}
$$

where $d_{i}$ are the Dynkin indices. We keep the cycles $\left\{\alpha_{5}, \ldots, \alpha_{8}\right\}$ zero size, so they correspond to the exceptional cycles of the $S U(5)$ singularity, but give a finite size to $\left\{\alpha_{-\theta}, \ldots, \alpha_{4}\right\}$. There is a remnant gauge symmetry, namely the Weyl group of $S U(5)_{H}$, which may be used to glue the latter

\footnotetext{
${ }^{10}$ For recent reviews of some of the issues in proton decay, see e.g., [73, 74].
} 
set of cycles together across local patches. This Weyl group, denoted $\mathscr{W}_{A_{4}}$, is generated by the reflections

$$
\left\{W_{\alpha_{-\theta}}, W_{\alpha_{1}}, \ldots, W_{\alpha_{3}}\right\}
$$

and plays the role of the structure group breaking $E_{8}$ to $S U(5)_{\mathrm{GUT}}$. As we vary the ALE over $S_{2}$, the roots undergo monodromies, and define a non-trivial local system (i.e., a flat bundle) over $S_{2}$.

To parameterize the sizes of the cycles, we introduce the dual basis $\omega_{i}$ of forms on the ALE such that $\int_{\alpha_{j}} \omega_{i}=\delta_{i j}$, and further the generator $\omega_{\theta}$ satisfying $\omega_{\theta}+d_{i} \omega_{i}=0$. Then on each local patch of $S_{2}$, the fibration is described by

$$
\left.\Omega(t)=\Omega_{0}+t_{i} \wedge \omega_{i}+\text { (higher order }\right)
$$

where $t_{i}$ is a $(2,0)$ form on the patch and $t_{i} \equiv 0$ for $i=5, \ldots, 8$ in order to enforce the $S U(5)$ singularity.

Although in a local patch on $S$ we can make an expansion (5.5), since globally the $t_{i}$ and $\omega_{i}$ undergo monodromies by the $\mathscr{W}_{A_{4}}$ Weyl group, it is not very convenient to work with them. A more convenient way to specify such a fibration is by working with the invariant polynomials. Let us first define

$$
\lambda_{1}=t_{4}, \quad \lambda_{2}=t_{4}+t_{3}, \ldots, \lambda_{5}=t_{4}+\cdots+t_{-\theta} .
$$

Then $\mathscr{W}_{A_{4}}$ acts as the symmetric group on the $\lambda_{i}$. Then to specify the fibration, we need to specify precisely five global holomorphic sections $\left\{a_{0}, a_{2}, a_{3}\right.$, $\left.a_{4}, a_{5}\right\}$. In our local patch they are given by

$$
a_{n} / a_{0}=\sigma_{n}\left(\lambda_{i}\right), \quad n=2, \ldots, 5,
$$

where $\sigma_{n}$ is the $n$th symmetric polynomial. The combination $\sigma_{1}\left(\lambda_{i}\right)$ is zero automatically due to the constraint $t_{-\theta}=-\sum d_{i} t_{i}$. The $a_{i} / a_{0}, i=2, \ldots, 5$ are the Casimirs of the VEV of the adjoint field $\Phi^{2,0}$ of the eight-dimensional gauge theory. It is not hard to see that the Chern classes of $\left\{a_{0}, a_{2}, a_{3}, a_{4}, a_{5}\right\}$ are given by $[13,14]$

$$
\left[a_{i}=0\right]=(6-i) c_{1}-t,
$$

where $c_{1}=c_{1}\left(T S_{2}\right)=-c_{1}\left(K_{S}\right)$ and $-t=c_{1}\left(N_{S}\right)$. 
Now let us write the Calabi-Yau four-fold more explicitly in terms of the $a_{i}$. We introduce variables $\{u, z, x, y\}$ of degrees $\{1,1,2,3\}$. Then we may write the sections as a single expression

$$
p_{5}=a_{0} z^{5}+a_{2} x z^{3}+a_{3} y z^{2}+a_{4} x^{2} z+a_{5} x y \text {. }
$$

The $d P_{8}$ fibration over $S$ is now simply given by the following equation:

$$
0=y^{2}+x^{3}+f x z^{4}+g z^{6}+u p_{5}(x, y) \text {. }
$$

To get an affine space, we can set $u \rightarrow 1$. Moreover, the terms $f x z^{4}$ and $g z^{6}$ are subleading and irrelevant for our purposes here, so we may set them to zero. Then we arrive at the equation of a deformed $E_{8}$ singularity:

$$
0=y^{2}+x^{3}+a_{0} z^{5}+a_{2} x z^{3}+a_{3} y z^{2}+a_{4} x^{2} z+a_{5} x y
$$

It is frequently desirable to keep the $d P_{8}$, but this is very similar and most of the comments in this section will go through.

The above data may also be represented by a spectral cover. The $d P_{8}$ fibration is equivalent to the spectral cover defined by $p_{5}=0$ in the elliptically fibered Calabi-Yau $y^{2}=x^{3}+f x+g$. In the limit that $f, g \rightarrow 0$, the $T^{2}$ fibration degenerates to a $\mathbf{P}^{1}$-fibration over $S_{2}$, and $z^{\prime}=y / x$ is identified with a coordinate on the $\mathbf{P}^{1}$. Then $p_{5}=0$ yields a spectral cover in the $\mathbf{P}^{1}$-fibration which is equivalent to the (compactified) $E_{8}$ ALE fibration. The spectral line bundle gets mapped to $G$-flux on $Y_{4}$. The locus where $\lambda_{i}=\lambda_{j}$ on the spectral cover for some $i \neq j$ is called the ramification locus (or ramification divisor, because it is complex codimension one). The spectral cover is smooth at this locus, but the vertical derivative vanishes. The projection of the ramification locus on $S$ is the branch locus. As we circle around this locus, the cycles undergo the monodromies by the Weyl group that we described above.

The adjoint representation of $E_{8}$ decomposes into the adjoint of $S U(5)_{H} \times$ $S U(5)_{\text {GUT }}$ plus some remaining representations, which can be read off from the Dynkin diagram:

$$
248=(\mathbf{2 4}, \mathbf{1})+(\mathbf{1}, \mathbf{2 4})+(\mathbf{5}, \mathbf{1 0})+(\overline{\mathbf{5}}, \overline{\mathbf{1 0}})+(\mathbf{1 0}, \overline{\mathbf{5}})+(\overline{\mathbf{1 0}}, \mathbf{5})
$$

The $E_{8}$ root $\alpha_{4}$ is a weight for the $(\mathbf{5}, \mathbf{1 0})$ representation of $S U(5)_{H} \times$ $S U(5)_{\text {GUT }}$ (since it extends the $S U(5)_{H}$ root system to $S U(6)$ and the $S U(5)_{\text {GUT }}$ root system to $S O(10)$ ), and acting with the remaining creation operators give the remaining weights of this representation. Similarly $-\alpha_{4}$ gives the $(\overline{\mathbf{5}}, \overline{\mathbf{1 0}}), 2 \alpha_{4}+\alpha_{3}+2 \alpha_{5}+\alpha_{6}+\alpha_{8}$ gives the $(\mathbf{1 0}, \overline{\mathbf{5}})$ 


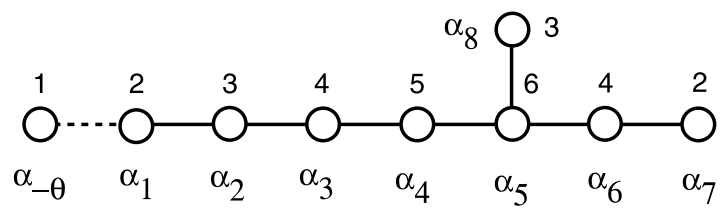

Figure 3: The extended $E_{8}$ Dynkin diagram and Dynkin indices.

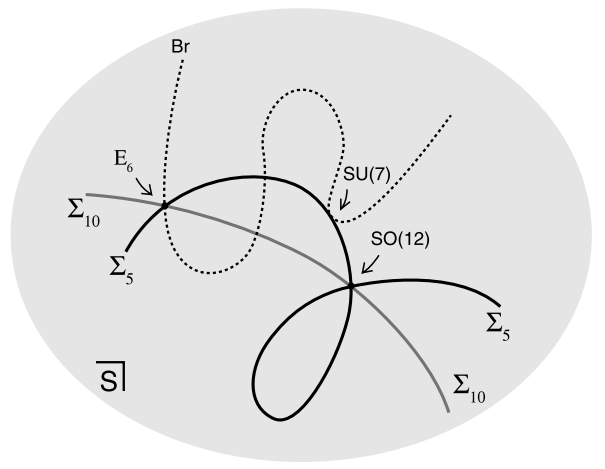

Figure 4: Schematic picture of the matter curves and branch locus on $S$, and their intersections, for generic values of the complex structure moduli.

and $-2 \alpha_{4}-\alpha_{3}-2 \alpha_{5}-\alpha_{6}-\alpha_{8}$ gives the $(\overline{\mathbf{1 0}}, \mathbf{5})$. For more details on the decomposition of the $E_{8}$ roots under $S U(5)_{H} \times S U(5)_{\mathrm{GUT}}$, see Appendix C.

Over a generic point on $S_{2}$, only $\left\{\alpha_{5}, \ldots, \alpha_{8}\right\}$ have zero size, and wrapping membranes (or rather $(p, q)$ strings) on these cycles gives the eightdimensional $S U(5)$ vector multiplet. However, over a complex codimension one locus on $S_{2}$, an extra cycle may shrink to zero, and wrapping a membrane on it gives rise to a hypermultiplet. When $a_{5}=0$, we have $\lambda_{i} \rightarrow 0$ for some $i$. Suppose we have $\lambda_{1} \rightarrow 0$. Then the cycle $\alpha_{4}$ shrinks to zero, and the singularity type is enhanced from $S U(5)_{\text {GUT }}$ to $S O(10)$, as we can easily read of from the Dynkin diagram in figure 3. In fact, as we can see from Appendix $\mathrm{C}$, when $\lambda_{1} \rightarrow 0$ there are actually twenty cycles shrinking to zero size simultaneously, precisely fitting in the $\mathbf{1 0} \oplus \overline{\mathbf{1 0}}$ of $S U(5)_{\mathrm{GUT}}$. Thus, the extra massless states fit in a hypermultiplet in the $\mathbf{1 0}$ of $S U(5)_{\mathrm{GUT}}$, and this locus this corresponds to the matter curve $\Sigma_{\mathbf{1 0}}$ :

$$
\Sigma_{\mathbf{1 0}}=\left\{a_{5}=0\right\}
$$

On the other hand, suppose $\lambda_{i}+\lambda_{j} \rightarrow 0$ for some $i \neq j$. Eg. suppose we have $\lambda_{1}+\lambda_{2} \rightarrow 0$. Then the cycle $\alpha_{3}+2 \alpha_{4}+2 \alpha_{5}+\alpha_{6}+\alpha_{8}$ shrinks to zero size, and computing the extended Dynkin diagram, we see that the 
singularity type is enhanced from $S U(5)_{\text {GUT }}$ to $S U(6)$. In fact according to Appendix $\mathrm{C}$, when $\lambda_{1}+\lambda_{2} \rightarrow 0$ there are actually ten cycles shrinking to zero size simultaneously, precisely fitting in the $\mathbf{5} \oplus \overline{\mathbf{5}}$ of $S U(5)_{\text {GUT }}$. Thus the extra vanishing cycles form a hypermultiplet in the $\mathbf{5}$ of $S U(5)_{\mathrm{GUT}}$, and this locus corresponds to the matter curve $\Sigma_{\mathbf{5}}$.

We can deduce an explicit equation for $\Sigma_{\mathbf{5}}$ as follows. When $\lambda_{i}+\lambda_{j} \rightarrow 0$ for $i \neq j$, it means that $p_{5}$ has solutions which are interchanged under $y \rightarrow$ $-y$. Equivalently if we write

$$
p_{5}=\left(a_{0}+a_{2} x+a_{4} x^{2}\right)+y\left(a_{3}+a_{5} x\right)=P(x)+y Q(x)
$$

then $P(x)$ and $Q(x)$ should have a common zero. This is measured by the vanishing of the resultant, so when

$$
\Sigma_{\mathbf{5}}=\left\{R_{8 c_{1}-3 t}=a_{0} a_{5}^{2}-a_{2} a_{3} a_{5}+a_{4} a_{3}^{2}=0\right\},
$$

we get the matter curve $\Sigma_{\mathbf{5}}$.

Now recall that the Yukawa couplings are given by the triple overlap of wave functions:

$$
\int_{S} \operatorname{Tr}\left(A^{0,1} \wedge A^{0,1} \wedge \Phi^{2,0}\right)
$$

where by the trace we just mean that one should pick a gauge singlet. This coupling is well-defined on Dolbeault cohomology classes. Since our wave functions have representatives that are localized on the matter curves, the Yukawa couplings get contributions only from the intersection of $\Sigma_{\mathbf{1 0}}$ and $\Sigma_{\overline{\mathbf{5}}}$. When these curves intersect, we have $a_{5}=0$ and $R=0$ so we must also have $a_{3}=0$ or $a_{4}=0$. If $a_{5}=a_{4}=0$, then up to Weyl reflections we can say that the cycles $\alpha_{4}$ and $\alpha_{3}$ shrink to zero and the singularity type is enhanced to $E_{6}$. If we wrap a membrane (or really $(p, q)$ string from the perspective of the IIb space-time) on the cycles $\alpha_{4}, \alpha_{4}+\alpha_{3}+2 \alpha_{5}+\alpha_{6}+\alpha_{8}$ and $-2 \alpha_{4}-\alpha_{3}-2 \alpha_{5}-\alpha_{6}-\alpha_{8}$, giving us states in the 10, 10 and $\mathbf{5}$ of $S U(5)_{\text {GUT }}$ respectively, then at the $E_{6}$ intersection point there is a relation in homology

$$
\left(\alpha_{4}\right)+\left(\alpha_{4}+\alpha_{3}+2 \alpha_{5}+\alpha_{6}+\alpha_{8}\right)+\left(-2 \alpha_{4}-\alpha_{3}-2 \alpha_{5}-\alpha_{6}-\alpha_{8}\right)=0
$$

Pictorially, this means that the three membranes wrapping these three cycles may be combined into a topologically trivial configuration. From the perspective of the IIb space-time, it means that on this intersection the ends of the three open $(p, q)$ strings can be combined into a gauge singlet. 
In terms of group theory, the statement (5.17) is equivalent to the fact that if we decompose the $\mathbf{7 8}$ of $E_{6}$ under $S U(5)_{\text {GUT }} \times U(1)_{a} \times U(1)_{b}$, which yields $\mathbf{1 0}_{1,1}+\mathbf{1 0}_{-1,1}+\overline{\mathbf{5}}_{0,2}+$ c.c plus additional neutral matter, then the $\operatorname{Tr}\left(\mathbf{7 8}^{3}\right)$ contains a contribution $\mathbf{1 0} \cdot \mathbf{1 0} \cdot \mathbf{5}$. Thus at such an intersection we get a contribution to the up type Yukawa coupling, inherited by KK reduction from the cubic coupling (5.16) in the eight-dimensional gauge theory:

$$
\left\{a_{5}=a_{4}=0\right\} \quad \rightarrow \quad \mathbf{1 0} \cdot \mathbf{1 0} \cdot \mathbf{5}
$$

Now consider the other type of intersection point, where $a_{5}=a_{3}=0$. Here we have (up to Weyl reflections) $\alpha_{4}=0$ and $\alpha_{9}=0$, where we define

$$
\alpha_{9}=\alpha_{2}+2 \alpha_{3}+2 \alpha_{4}+2 \alpha_{5}+\alpha_{6}+\alpha_{8}
$$

Note that $\alpha_{9}$ is a weight for the $(\mathbf{1 0}, \overline{\mathbf{5}})$ of $S U(5)_{H} \times S U(5)_{\text {GUT }}$, with size $\lambda_{2}+\lambda_{3}$, and by acting with raising and lowering operators we can get the remaining states in this representation. Computing the inner products, we see that the Dynkin diagram of $\left\{\alpha_{4}, \ldots, \alpha_{9}\right\}$ is that of $S O(12)$, so the singularity type is enhanced to $S O(12)$. Now note that $\alpha_{4}+\alpha_{9}$ is still a root of $S O(12)$, so we can make gauge invariant combinations of the type $\mathbf{1 0} \cdot \overline{\mathbf{5}} \cdot \overline{\mathbf{5}}$, yielding the down type Yukawas:

$$
\left\{a_{5}=a_{3}=0\right\} \quad \rightarrow \quad \mathbf{1 0} \cdot \overline{\mathbf{5}} \cdot \overline{\mathbf{5}}
$$

The curve $R=0$ clearly has a double point singularity at this intersection point. As pointed out in [17], if we keep track of the vanishing cycles along each branch, we see that the charged open strings, and hence the hypermultiplet in the $\mathbf{5}$, actually naturally propagate on the normalization of the matter curve, i.e., they propagate on the resolution obtained by replacing each double point by two distinct points.

This accounts for all the intersections of $\Sigma_{\mathbf{5}}$ and $\Sigma_{\mathbf{1 0}}$. However there is another type of singularity enhancement that occurs generically at codimension two on $S$ but does not correspond to the intersection points above. Let us define the root

$$
\tilde{\alpha}_{8}=\alpha_{3}+2 \alpha_{4}+3 \alpha_{5}+2 \alpha_{6}+\alpha_{7}+2 \alpha_{8} .
$$

This is one of the weights of the $(\mathbf{1 0}, \overline{\mathbf{5}})$ representation of $S U(5)_{H} \times$ $S U(5)_{\text {GUT }}$, with size $\lambda_{1}+\lambda_{2}$. Now suppose that $\tilde{\alpha}_{8}$ and $\alpha_{9}$ vanish at the same time. The Dynkin diagram generated by $\left\{\tilde{\alpha}_{8}, \alpha_{8}, \alpha_{5}, \alpha_{6}, \alpha_{7}, \alpha_{9}\right\}$ is that 
of $S U(7)$. Now let us apply to $\alpha_{9}$ the raising operator corresponding to the highest root of $S U(5)_{\mathrm{GUT}}$, yielding:

$$
\tilde{\alpha}_{9}=\alpha_{2}+2 \alpha_{3}+2 \alpha_{4}+3 \alpha_{5}+2 \alpha_{6}+\alpha_{7}+2 \alpha_{8} .
$$

The difference between these two roots is

$$
\tilde{\alpha}_{8}-\tilde{\alpha}_{9}=-\alpha_{2}-\alpha_{3}
$$

with size $\lambda_{1}-\lambda_{3}$. Hence we see that $\alpha_{2}+\alpha_{3}$ is also a vanishing two-cycle, but if we wrap a membrane on it we get a hypermultiplet $N$ which is neutral under $S U(5)_{\text {GUT }}$. This corresponds to one of the non-normalizable complex structure moduli describing deformations of the 7-branes. Indeed $\alpha_{2}+\alpha_{3}$ is a root for $S U(5)_{H}$, so we see that $N$ transforms in the adjoint of $S U(5)_{H}$. In the heterotic string, it would be identified with a vector bundle modulus. Note that complex structure moduli like $N$ are not localized along any matter curve - they are supported in the bulk.

To arrange such an $S U(7)$ point, we need (up to Weyl reflections) that $\lambda_{1}+\lambda_{2}=\lambda_{2}+\lambda_{3}=0$, but $\lambda_{1}, \lambda_{3} \neq 0$, because that would impose three conditions and so will not happen generically at codimension two. Thus this corresponds to some intersection of the branch locus with $\Sigma_{\mathbf{5}}$. However, not all intersections of the branch locus with $\Sigma_{5}$ are $S U(7)$ points, so how do we distinguish them geometrically?

Let us enumerate the possible intersections between $\Sigma_{\mathbf{5}}$ and the branch locus. One such type of intersection corresponds to $\lambda_{i}=\lambda_{j}=0$ for $i \neq j$. As we saw above, these are the $E_{6}$ enhancement points where the top quark Yukawa couplings are localized, and $\Sigma_{\mathbf{5}}$ also intersects $\Sigma_{\mathbf{1 0}}$ there. We could also have $\lambda_{1}+\lambda_{2}=0$ intersecting with the locus where $\lambda_{i}-\lambda_{j}=0$, but both $i$ and $j$ are not equal to one or two. This is generically a transverse intersection and there is no singularity enhancement at the intersection; lifting to the spectral cover, the ramification locus does not even intersect the zero section here. Even when the intersection between $\Sigma_{\mathbf{5}}$ and the branch locus is accidentally not transversal, we can make it transversal by a small complex structure deformation. Finally we could have the $S U(7)$ points where $\lambda_{1}+\lambda_{2}=0$ and $\lambda_{i}-\lambda_{j}=0$, and precisely one of $i$ or $j$ is equal to one or two (they should not both be equal, as this would give us back the $E_{6}$ intersection points). A simple local model near such an enhancement point is given by the two-fold spectral cover

$$
z_{1}+z_{2} s+s^{2}=0
$$

where $z_{1}, z_{2}$ are local coordinates on $S$, and $s$ is a local coordinate on the canonical bundle of $S$. In this model, $\Sigma_{\mathbf{5}}$ is given by $z_{1}=0$ and the branch 
locus is given by $z_{2}^{2}-4 z_{1}=0$. Although both curves are smooth, we see that the intersection is not transversal: the branch locus becomes tangential to $\Sigma_{\mathbf{5}}$ at the intersection $z_{1}=z_{2}=0$, where the singularity is enhanced to $S U(7)$. The non-transversality is required by the analytic structure and cannot be removed by a small deformation (as this would just amount to a redefinition of $z_{1}$ and $z_{2}$ ). Hence such intersection points can be distinguished geometrically.

From the homology relation (5.23), we see that we can make a gauge invariant coupling:

$$
\left\{R=B r=0, \operatorname{det}\left(R^{\prime}, B r^{\prime}\right)=0\right\} \quad \rightarrow \quad \mathbf{5} \cdot \overline{\mathbf{5}} \cdot \mathbf{1}
$$

where $B r=0$ denotes the branch locus of the spectral cover. ${ }^{11}$ Of course getting a massless $\mathbf{5} / \overline{\mathbf{5}}$ pair localized on a connected component of $\Sigma_{\mathbf{5}}$ generally requires some fine-tuning of the complex structure moduli, and such pairs are easily lifted. Turning on an expectation value for $N$ yields a mass term for the hypermultiplet. This corresponds to deforming the branch locus and $\Sigma_{\mathbf{5}}$ on $S$.

The $S U(7)$ enhancement points are on different footing from the $E_{6}$ and $S O(12)$ points; they are generally smooth points of $\Sigma_{\mathbf{5}}$ and it seems unlikely that the $\mathbf{5} \cdot \overline{\mathbf{5}} \cdot \mathbf{1}$ couplings are localized there. Further, one may also get $\mathbf{1 0}$. $\overline{\mathbf{1 0}} \cdot \mathbf{1}$ couplings, and it does not appear to require singularity enhancements.

\subsection{Extra $U(1)$ 's and their $D$-terms}

In the $S U(5)$ GUT models $R$-parity is not guaranteed. In order to prevent couplings of the type $\mathbf{1 0}_{m} \cdot \overline{\mathbf{5}}_{m} \cdot \overline{\mathbf{5}}_{m}$, we have to somehow be able to distinguish between $\overline{\mathbf{5}}_{h}$ and $\overline{\mathbf{5}}_{m}$ zero modes. This can be achieved by localizing the $\overline{\mathbf{5}}_{h}$ and $\overline{\mathbf{5}}_{m}$ on different matter curves, charging them differently under an extra gauge symmetry, or both.

The idea of using $U(1)$ symmetries has long been discussed in the heterotic literature, and is implicit in the construction of bundles by extension. As already noted in [77] the discussion can be largely translated to $F$-theory. In the discussion of the previous section we assumed that the spectral cover is smooth and irreducible, in which case the holonomy group of the spectral

\footnotetext{
${ }^{11}$ One may also write an explicit equation for the branch locus: it corresponds to the discriminant, here given by the resultant of $p_{5}$ and its vertical derivative. E.g., for the two-fold cover in (5.24) we get $\operatorname{Res}\left(z_{1}+z_{2} s+s^{2}, z_{2}+2 s\right)=4 z_{1}-z_{2}^{2}$.
} 
cover breaks $E_{8}$ to $S U(5)_{\mathrm{GUT}}$. However if the spectral cover degenerates, under certain conditions one may get additional gauge symmetries.

The five-fold spectral cover can be degenerated in various ways. When the spectral cover becomes reducible, for instance if the sections $a_{i}$ are such that we have a $3+2$ factorization

$$
p_{5}=\left(b_{0}+b_{2} x\right)\left(d_{0}+d_{2} x+d_{3} y\right)
$$

with $b_{i}, d_{j}$ generic, then the structure group of the spectral cover is reduced from $\mathscr{W}_{A_{4}}$ to $\mathscr{W}_{A_{1}} \times \mathscr{W}_{A_{2}}$. In order to understand the low energy gauge group, we also need to know what happens with the gauge field on the intersection of the reducible pieces. A rank one sheaf on the reducible surface consists of a pair of line bundles on the reducible components, together with a gluing morphism on the intersection $[23,70]$. If the gluing morphism vanishes identically, then there is an extra $U(1)_{X}$ symmetry that commutes with the structure group; in fact in this example there may be additional massless $W$-bosons, and the GUT group is enhanced to $S U(6)$, but this is typically broken to $S U(5)_{\text {GUT }} \times U(1)_{X}$ through $U(1)_{X}$ flux. If the elliptic fibration admits a second section, then we can also break to $S U(5)_{\mathrm{GUT}} \times U(1)_{X}$ by using an abelian Higgs field. At the intersection of the two reducible pieces of the spectral cover, one finds chiral fields charged under $U(1)_{X}$, whose internal zero modes correspond to the gluing morphism mentioned above. If the $D$-terms for $U(1)_{X}$ can be satisfied so that these charged moduli have zero VEVs, then we have a candidate for an extra $U(1)_{X}$ symmetry.

The $U(1)_{X}$ could still be lifted by other effects. In Section 2 , we saw that the $U(1)_{X}$ also gets a contribution to its mass from its couplings to axions, if the matrices

$$
\Pi_{M}^{X}=\int_{Y_{4}} \beta_{M} \wedge \omega^{X} \wedge G
$$

are non-zero. It is also possible that $\omega^{X}$ may not be extended globally. Thus the fate of the extra $U(1)$ can not be completely understood from the spectral cover for the local model alone.

In the supergravity approximation, we expect the Fayet-Iliopoulos parameters to be proportional to $G \wedge J \sim F \wedge J$. From the analysis of Becker and Becker we know that if we are working within $11 d$ supergravity on a smooth resolved Calabi-Yau, then we must have $J \wedge G=0$, but this need not be true in the $F$-theory limit, as their may be new light fields entering the $D$-term potential. To understand the dependence of the masses on the moduli, we need to write the $D$-term potential more precisely. We now describe 
how to do this in the four-fold picture. A brief summary of the conclusions here was already included in [23], in relation to $M 5$-instantons.

To find the $D$-terms in a supersymmetric manner, it is convenient to dualize the Green-Schwarz couplings. The Kähler potential for the Kähler moduli is

$$
\mathcal{K}=M_{P l}^{2} \hat{\mathcal{K}}=-2 M_{P l}^{2} \log \frac{1}{6} m_{10}^{6} \int_{B_{3}} J^{3} .
$$

The Kähler moduli are defined as

$$
T_{a}=\frac{1}{2} m_{10}^{4} \int_{D_{a}} J \wedge J-i \int_{D_{a}} C_{4}
$$

Then we may rewrite

$$
\mathcal{K}(T) \sim-\frac{1}{2} M_{P l}^{2} \log \frac{8}{6}\left(\operatorname{Re} T_{a}\right)\left(\operatorname{Re} T_{b}\right)\left(\operatorname{Re} T_{c}\right) d^{a b c},
$$

where $d_{a b c}=D_{a} \cap D_{b} \cap D_{c}$, and indices are raised and lowered using the metric $\hat{\mathcal{K}}_{a b}$.

In the presence of 7-branes, the RR field $C_{(4)}$ obtains an anomalous transformation law:

$$
\delta C_{(4)} \sim \sum_{m} \operatorname{Tr}\left(\Lambda_{m} F_{m}\right) \wedge \delta^{(2)}\left(D_{m}\right)-\operatorname{Tr}(\Theta R) \wedge \delta^{(2)}\left(D_{m}\right)
$$

This transformation law is determined by anomaly cancelation. We can try to write this in terms of four-fold data as follows: $C_{(4)}$ gets promoted to $C_{(6)}$ with two indices on the elliptic fiber, the $F_{m}$ get lifted to $G$-flux, and the $\Lambda_{m}$ get lifted to a harmonic two-form $\Lambda=\Lambda_{m} \omega^{m}$. Then we are motivated to write the variation under gauge transformations as

$$
\delta C_{(6)} \sim G \wedge \Lambda+I_{6}(R, \Theta)
$$

One can derive this more directly from the $M$-theory perspective, which is the proper way to do it. Due to the Chern-Simons term in 11d supergravity, 
one finds that

$$
d^{2} C_{6}+\frac{1}{2} G \wedge G+I_{8}(R)=0
$$

By descent we then arrive at (5.32). Now the Kähler moduli are not invariant under a gauge transformation of $U(1)_{X}$ :

$$
\operatorname{Im}\left(T_{a}\right) \rightarrow \operatorname{Im}\left(T_{a}\right)+q_{a}^{X} \lambda_{X}
$$

Therefore, the Kähler potential for the moduli is modified to

$$
\hat{\mathcal{K}}\left(T_{a}+T_{a}^{\dagger}\right) \rightarrow \hat{\mathcal{K}}\left(T_{a}+T_{a}^{\dagger}-q_{a}^{X} V_{X}\right)
$$

so that under a gauge transformation, the action is invariant. This Kähler potential now includes the dualized Green-Schwarz couplings, which are of the form $q_{a}^{X} A_{\mu}^{X} \partial^{\mu} \operatorname{Im}\left(T_{a}\right)$, in a manifestly supersymmetric manner. The Fayet-Iliopoulos term is given as the coefficient of $\int d^{4} \theta V_{X}$, i.e.,

$$
\xi^{X}=\left.M_{P l}^{2} \frac{\partial \hat{\mathcal{K}}}{\partial V_{X}}\right|_{V=0} \sim M_{P l}^{2} \frac{q_{a}^{X}\left(\operatorname{Re} T_{b}\right)\left(\operatorname{Re} T_{c}\right) d^{a b c}}{\left(\operatorname{Re} T_{a}\right)\left(\operatorname{Re} T_{b}\right)\left(\operatorname{Re} T_{c}\right) d^{a b c}}
$$

One can also understand this as the moment map associated to the gauged isometry (5.34). The $D$-term potential is given by

$$
V_{D}=\frac{1}{2} g_{X}^{2}\left(\xi^{X}-\sum q_{\phi}^{X}|\phi|^{2}\right)^{2}
$$

where $\phi$ are recombination moduli or matter fields with charge $q_{\phi}^{X}$ under $U(1)_{X}$. More generally if there are multiple $U(1)$ 's, there could be mixing and we should replace $g_{X}^{2}$ by the inverse of the holomorphic gauge kinetic function $\operatorname{Re}(f)$. It follows that we only need to determine the precise coupling $q_{a}^{X}$. This can be read off from (5.31) or (5.32) and the definition of the Kähler moduli. From (5.32) we read off that

$$
q_{a}^{X} \sim \int_{Y_{4}} G \wedge \omega^{X} \wedge P\left(\pi_{Y_{4}}^{*} D_{a}\right)
$$

The symbol $P$ is meant to represent the Poincaré dual. Defining parameters $t^{i}$ by $J=t^{i} P\left(D_{i}\right)$, we claim that $\left(\operatorname{Re} T_{j}\right)\left(\operatorname{Re} T_{k}\right) d^{i j k} \sim \operatorname{vol}\left(B_{3}\right)^{3} t^{k}$. Substituting in (5.36), we find that

$$
\xi^{X} \sim m_{10}^{4} \int_{Y_{4}} G \wedge \omega^{X} \wedge J
$$


where we used $M_{P l}^{2} \sim m_{10}^{8} \operatorname{vol}\left(B_{3}\right)$. This is expected, because it is related by SUSY to the matrices $\Pi_{M}^{X}$ discussed earlier which describe the coupling between $U(1)_{X}$ gauge fields and $\mathrm{RR}$ axions. We would like to use this in a regime which is far form the $11 d$ supergravity limit, where we derived this expression (and where the equations of motion yield $\mathrm{G} \wedge J=0$ ). The nonrenormalization theorem for Fayet-Iliopoulos terms gives us some confidence that it can be extrapolated to $F$-theory. Furthermore, the kinetic terms of extra $U(1)$ 's typically scale as

$$
\frac{1}{g_{X}^{2}} \sim \int_{Y_{4}} \omega_{X} \wedge * \omega_{X} \sim m_{10}^{3} \operatorname{vol}\left(B_{3}\right)^{1 / 2}
$$

Here we used the relation between $M$ - and $F$-theory to convert the volume of $Y_{4}$ measured in eleven-dimensional Planck units to the volume of $B_{3}$ measured in ten-dimensional Planck units. Note that such gauge fields are generally not localized on the cycle wrapped by the GUT brane, so the scaling is not set by $1 / R_{S}$. In the non-compact limit they freeze out, which further shows that their fate depends on the UV completion.

Note that the charged fields $\phi$ in (5.37) whose $D$-terms could possibly compensate for a non-zero Fayet-Iliopoulos term are obtained by quantizing wrapped $M 2$-branes. They are very heavy and have been integrated out in $11 d$ supergravity, and cannot be condensed. This is consistent with the fact that $\mathrm{G} \wedge J=0$ in $11 d$ supergravity. Extrapolating to $F$-theory by varying Kähler moduli, these states becomes light and should be included in the effective action. Even so, the effective action describes the linearized deformations, so the Fayet-Iliopoulos parameter must be small in this regime, so that all the masses of the fields in the effective action are parametrically below the KK scale.

In particular, using the above expressions for generic moduli dependence and non-zero Fayet-Iliopoulos term we find that the masses squared scale like $g_{X}^{2} \xi^{X} \sim 1 / R_{B_{3}}$, which exceeds the KK scale. Even with the scaling $g_{X}^{2} \sim \operatorname{vol}\left(B_{3}\right)^{-2 / 3}$ seen in type IIb (where such $U(1)$ gauge symmetries can be cleanly localized on four-cycles), the masses are not suppressed compared to the KK scale. Under these circumstances a consistent effective Lagrangian with a $U(1)_{X}$ symmetry requires the $\xi^{X}$ to vanish identically, or else we are expanding around the wrong background. This applies for example to models with only a single Kähler modulus. However when there are multiple Kähler moduli one may be able to tune some ratio to get a parametrically small VEV, although one still has to ensure that all effective cycles of $B_{3}$ are large compared to the $10 d$ Planck scale when we do this tuning. 
Since we extrapolated expressions from $11 d$ supergravity, one may wonder if we should trust this at all. Let us look at possible corrections to $\xi^{X}$. The non-renormalization theorem for the Fayet-Iliopoulos term says that there could be a perturbative correction at one loop. In the context of the heterotic string, it is well-known that for an anomalous $U(1)_{X}$ there could be a one-loop contribution that is quadratically divergent. In the context of perturbative type II, the evidence seems to indicate that such a correction is absent if tadpoles are canceled (by open/closed duality). Furthermore, under heterotic/F-theory duality it appears that the heterotic one-loop contribution gets mapped to a tree level contribution (see Appendix $\mathrm{C}$ of [15]). It also matches qualitatively with the Higgs bundle picture [70], though a precise match requires a more detailed analysis of the degeneration limit involved.

Even if extra $U(1)$ symmetries are unbroken perturbatively, they may still be violated by non-perturbative effects. Consider the action of a D3instanton wrapping a four-cycle $D$ :

$$
e^{-\operatorname{vol}(D)+i \int_{D} C_{(4)}}
$$

From (5.31) we see that the instanton action shifts under $\Lambda_{m}$ when either (1) the worldvolumes coincide $\left(D_{m}=D\right)$ and $\int_{D} F_{m} \wedge c_{1}(K) \neq 0$, or (2) the worldvolumes intersect $\left(D \cap D_{m}=\Sigma_{m}\right)$ and $\int_{\Sigma_{m}} F_{m} \neq 0$. (Again this implicitly uses a weakly coupled IIb picture and is slightly imprecise, it is better to use (5.32) and obtain $q_{D}^{X}$ as in (5.38).) In these cases, the D3instanton can generate superpotential terms which violate the $U(1)$ symmetry, by an amount equal and opposite to that of the instanton action. For instance in the case of an extra $U(1)_{B-L}$ symmetry, we might generate terms of the form

$$
\mathscr{L} \supset d^{2} \theta(Q D L+L E L+D U D) f(m) \mathrm{e}^{-V_{D 3}+\mathrm{i} \int_{D 3} C_{\mathrm{RR}}^{(4)}}
$$

where $f(m)$ is a one-loop determinant depending on complex structure moduli, but not on Kähler moduli. Such effects are exponentially suppressed and so we have a good approximate symmetry. To understand how instanton induced corrections to holomorphic couplings of charged fields such as (5.42) get generated requires a more in depth analysis of the chiral two-form on the M5-brane, in particular of certain correlation functions of this field. This is discussed in [79]. See also [24].

As an aside, F-theory has fewer anomalous $U(1)$ 's than perturbative IIb, and thus many more instantons contributing to the superpotential [79]. In particular, it has been argued that in type IIb, a D3 instanton wrapped on the Standard Model cycle (or intersecting it) cannot contribute to the 
superpotential [80]. This is because $C_{4}$ has an anomalous transformation under the $U(1) \subset U(5)$, proportional to the number of generations. To compensate for this, one has to consider derivatives of the superpotential, which effectively puts charged fields in front of the exponentiated instanton action. In $F$-theory by contrast, there is no such $U(1)$ with a mass small compared to the KK scale, and so the effective action contains only the $S U(5)$ gauge fields and does not respect this $U(1)$ symmetry. Therefore such an instanton can contribute to the superpotential in F-theory [79]. Lifting of instanton zero modes for finite coupling is also familiar in field theory ${ }^{12}$. A similar story also holds in IIa: when we lift to $M$-theory, all anomalous $U(1)$ 's have masses at or above the KK scale $[12,78]$.

Given a five-fold spectral cover, there is only a limited number of ways in which we can degenerate the cover to gain additional $U(1)$ symmetries. The possible degenerations and the resulting $U(1)_{X}$ symmetries were discussed in [77]. The simplest possibilities are a $4+1$-split, which leads to an extra $U(1)_{B-L}$ symmetry, and a $3+2$-split, which leads to an $S U(6)_{\text {GUT }}$ group (typically broken to $\left.S U(5)_{\mathrm{GUT}} \times U(1)_{X}\right)$.

The extra $U(1)$ symmetries could be used for various phenomenological purposes, such as for preventing rapid proton decay, or for generating flavor structure, as already advocated in the context of heterotic models. In one scenario, the $U(1)$ selection rules are maintained in perturbation theory. Then the instanton action $\mathrm{e}^{-\mathrm{vol}}$ plays the role of the Froggatt-Nielsen field. A second scenario is to explicitly break the $U(1)$ symmetry, but preserve some of its selection rules through the holomorphic zero mechanism.

\subsection{Proton decay operators of dimension four and five}

We would like to have the benefits of some of the possible extra $U(1)$ symmetries, without some of their drawbacks. We propose to do this as follows. When we split the spectral cover to gain an extra $U(1)$, we also break up the matter curves, and multiplets with different $U(1)_{X}$ charges get localized in different ways. The perturbative vanishing of superpotential couplings violating these symmetries now comes about because overlap integrals vanish due to the localization of the wave functions. The crucial ingredient here however is localization properties of the wave functions of matter fields, rather than the $U(1)_{X}$ symmetry, so we can imagine splitting up the matter curves without splitting up the spectral cover itself. Localization would still forbid suitable couplings, but not lead to a preserved $U(1)_{X}$ symmetry that forbids too many couplings. Similar ideas have been advocated in the

\footnotetext{
${ }^{12}$ We would like to thank Chris Beasley for emphasizing this.
} 
phenomenology literature on extra dimensions for a while, although one of the difference with that literature is that due to the holomorphic nature of superpotential calculations, the effect of localization of the wave functions is much more pronounced here.

Let us consider an $S U(5)_{\text {GUT }}$ model. Clearly for a generic choice of sections, the matter curves will be irreducible. Hence if we want $\Sigma_{\overline{\mathbf{5}}}$ to be reducible, we must require $R$ to factorize into two global holomorphic sections, $R_{8 c_{1}-3 t}=b_{8 c_{1}-3 t-q} b_{q}$ for some $q \in H^{2}\left(S_{2}\right)$. Note this does not necessarily mean that $\Sigma_{\mathbf{1 0}}$ also factorizes. We will take $\Sigma_{\overline{\mathbf{5}}_{m}}$ to correspond to $b_{8 c_{1}-3 t-q}=0$, and we will further factorize $b_{q}=b_{u} b_{d}$ so that $b_{u}=0$ and $b_{d}=0$ correspond to $\Sigma_{\mathbf{5}_{h}}$ and $\Sigma_{\overline{\mathbf{5}}_{h}}$ respectively (it will be clear later why this is needed).

Therefore one solution to eliminate dimension four proton decay is as follows: we must factorize $R$ as above, and in order to eliminate the $\mathbf{1 0}_{m}$. $\overline{\mathbf{5}}_{m} \cdot \overline{\mathbf{5}}_{m}$ Yukawa coupling we must ensure that $a_{5}=b_{8 c_{1}-3 t-q}=0$ implies that also $b_{d}=0$. This is just saying that all down type couplings correspond to $\mathbf{1 0}_{m} \cdot \overline{\mathbf{5}}_{m} \cdot \overline{\mathbf{5}}_{h}$ Yukawa couplings. Or if we are content with eliminating the down type Yukawas at tree level, we could simply require that $\Sigma_{\mathbf{1 0}_{m}}$ and $\Sigma_{\overline{\mathbf{5}}_{m}}$ do not intersect at all. Such a configuration still allows for up type Yukawa couplings. The latter option actually seems somewhat attractive since only the top quark Yukawa coupling is experimentally observed to be of order one.

Now we come to the dimension five operators, i.e., the quartic terms in the superpotential. Let us first make some general remarks about quartic (and higher order) terms in the superpotential. A straightforward dimensional reduction of the eight-dimensional Yang-Mills theory only yields cubic interaction vertices in the superpotential (5.16). So in order to get a quartic interaction in the low energy effective theory valid below the KK scale, we have to take two cubic vertices and connect them by a propagator of a mode with mass at least the KK scale. Starting with the eight-dimensional YangMills theory, the only modes that can appear in the intermediate channel are the KK modes of the eight-dimensional theory and the matter hypermultiplets. Corrections to the eight-dimensional Yang-Mills theory from more massive modes could give further contributions, but would also be relatively suppressed, and probably decouple in the $M_{P l} \rightarrow \infty$ limit. Summing the propagators of all the massive KK modes yields the Green's function $G_{\bar{\partial}}$ of the $\bar{\partial}$ operator. Thus a four-point coupling in the superpotential is recovered by computing an amplitude of the form

$$
\int_{S}\left\langle\psi_{1} \wedge \psi_{2} G_{\bar{\partial}} \psi_{3} \wedge \psi_{4}+\psi_{2} \wedge \psi_{3} G_{\bar{\partial}} \psi_{4} \wedge \psi_{1}\right\rangle
$$


where $\psi \sim A^{0,1}+\Phi^{2,0}$ is the Dolbeault cohomology class paired with a fourdimensional massless chiral field. In other words, superpotential terms are computed only from the fermionic part of the eight-dimensional gauge theory, i.e., from the holomorphic Chern-Simons theory [88]. The quartic terms in the superpotential correspond to a (length three) Massey product. Since they express topological information, they can also be computed in alternative ways that are manifestly independent of the Kähler moduli [89]. However in the following it will be useful to keep the picture with intermediate propagating KK modes in mind.

In four-dimensional models, the dimension five operators $d^{2} \theta Q Q Q L$ and $d^{2} \theta U D U E$ leading to proton decay can be generated by exchange of massive Higgsino triplets. If present, they lead to the decay $p \rightarrow K^{+} \bar{\nu}$ with a lifetime that is hard to reconcile with experiment, at least in the more minimal versions of supersymmetric GUTs. In F-theory models there is a natural solution to the doublet-triplet splitting problem, which basically eliminates the triplet partners of the Higgses. However there are still massive KK modes with the same quantum numbers (charged in the representation $(\mathbf{1}, \overline{\mathbf{3}})_{1 / 3}$ and its conjugate) supported on the matter curves. Their exchange would lead to the same dimension five operators suppressed only by a single power of $M_{\mathrm{KK}}$, which is slightly below $M_{\mathrm{GUT}}$, and thus leads to essentially the same problems as in four-dimensional models. So we would like to suppress the leading contributions to these operators. (There are of course other scenarios than we discuss; for example in split SUSY, dimension five proton decay is also suppressed, due to large masses for the squarks).

Massive fields in the $(\mathbf{1}, \overline{\mathbf{3}})_{1 / 3}$ representation naturally propagate on any of the matter curves which support a $\mathbf{5}$ or $\overline{\mathbf{5}}$, i.e., curves which support $\left(L, d^{c}\right)$, the Higgses and possibly messenger fields for SUSY breaking. This means such dimension five operators are generically present and we have to work to avoid them. If we want $\mathbf{1 0}_{m} \cdot \overline{\mathbf{5}}_{m} \cdot \overline{\mathbf{5}}_{h}$ down type Yukawa couplings then there will be interactions of the form

$$
Q L T_{d}+U D T_{d}
$$

where $T_{d}$ is a massive KK triplet supported on $\Sigma_{\overline{\mathbf{5}}_{h}}$. Now further since we have $\mathbf{1 0}_{m} \cdot \mathbf{1 0}_{m} \cdot \mathbf{5}_{h}$ up type Yukawa couplings, there will also be couplings of the form

$$
Q Q T_{u}+U E T_{u}
$$

where $T_{u}$ is a massive $\mathrm{KK}$ triplet supported on $\Sigma_{\mathbf{5}_{h}}$. 
Thus our options seem to be (1) arrange the matter curves so that a mass term of the form $m T_{u} T_{d}$ is forbidden, (2) engineer some $U(1)$ symmetry so that the dimension five operators are forbidden, or (3) arrange the matter curves so that the down type Yukawas, and hence also $Q L T_{d}+U D T_{d}$ are classically forbidden. The third scenario was already discussed in the context of eliminating the dimension four operators, however on its own it now seems less appealing. Whatever quantum effect would end up generating the down type Yukawas of the right order of magnitude would presumably also end up generating $Q Q T_{u}+U E T_{u}$ with coefficients that are too large, unless we also eliminate $m T_{u} T_{d}$ mass terms. Hence we concentrate on the first two scenarios.

For the first scenario, we must make sure that either $\Sigma_{\mathbf{5}_{h}}$ and $\Sigma_{\overline{\mathbf{5}}_{h}}$ do not intersect, or if they do it must be a type of intersection that does not allow for a $\mathbf{5} \cdot \overline{\mathbf{5}}$ mass term. This is the reason why in the above we required the Higgs curve to factorize as $b_{q}=b_{u} b_{d}$, and as we see we actually need a slightly stronger condition on the allowed intersections. It is also the same condition that would eliminate the classical $\mu$ term, so we see that these two issues are connected. Note though that whether or not $\Sigma_{\overline{\mathbf{5}}_{m}}$ and $\Sigma_{\overline{\mathbf{5}}_{h}}$ intersect, we may still get Giudice-Masiero operators of type $H_{u} H_{d} S^{\dagger}$ where $S$ is a complex structure modulus, because this is a term in the Kähler potential rather than a term in the superpotential. If the curves do not intersect, the overlap between the Gaussian tails of the wave functions gives a suppression of order $\exp \alpha_{G}^{-1 / 2} \sim \mathrm{e}^{-5}$, since $\alpha_{G}^{-1 / 4}$ is the typical distance between non-intersecting matter curves.

Perhaps surprisingly, the equation of the matter curve $\Sigma_{\mathbf{5}}$ naturally allows for factorizations satisfying all these requirements. For instance we can take

$$
\Sigma_{\mathbf{5}_{h_{u}}}=\{h=0\}, \quad a_{4}=\alpha_{4} h, \quad a_{2}=\alpha_{2} h, \quad a_{0}=\alpha_{0} h .
$$

Then we factorize

$$
0=a_{0} a_{5}^{2}-a_{2} a_{3} a_{5}+a_{3}^{2} a_{4}=\underbrace{h}_{\Sigma_{\mathbf{5}_{h_{u}}}} \underbrace{\left(\beta_{1} a_{5}+\alpha_{4} a_{3}\right.}_{\Sigma_{\overline{\mathbf{5}}_{h_{d}}}}) \underbrace{\left(\beta_{2} a_{5}+a_{3}\right)}_{\Sigma_{\overline{\mathbf{5}}_{m}}},
$$

where $\alpha_{0}=\beta_{1} \beta_{2}, \alpha_{2}=-\beta_{1}-\alpha_{4} \beta_{2}$. If we can localize the MSSM fields on the indicated curves, then there is no dimension four proton decay because we eliminated the double points on each irreducible piece of $\Sigma_{\mathbf{5}}$. (More precisely, we also have to make sure that the spectral sheaf decomposes, i.e., there is no gluing morphism). If we take $\alpha_{4}=1$ and arrange all three pieces of $\Sigma_{\mathbf{5}}$ to be non-intersecting save for the down type Yukawa intersections $a_{3}=a_{5}=0$, then there is also no dimension five proton decay 
and the mu-term is absent. This happens despite the absence any extra $U(1)$ symmetries, because the spectral cover subject to these conditions is generally still irreducible. Moreover if the curves are non-intersecting apart from the required Yukawa couplings, then there are no infinitesimal deformations that could recombine the curves. Therefore the absence of the undesired operators is not dependent on moduli stabilization or fine tuning. ${ }^{13}$

One should be careful with the fluxes however. In order to get only an $H_{u}$ on $\Sigma_{H_{u}}$ and an $H_{d}$ on $\Sigma_{H_{d}}$, we want a non-zero hypercharge flux through these components. As we discussed earlier, the hypercharge flux is always orthogonal to homology classes dual to linear combinations of $c_{1}$ and $t$, so the equation for $\Sigma_{H_{u}}$ cannot simply be a polynomial in the $a_{i}$. If we appropriately factor the $a_{i}$ (restricted to $S$ ), then in principle we can evade this constraint. It is not hard to see that with the above expressions, the hypercharge flux through $\Sigma_{H_{u}}$ and $\Sigma_{H_{d}}$ must be equal and opposite. In the above factorization this requires $\alpha_{4} \neq 1$, and thus reintroduces dimension five proton decay through massive triplets propagating on $\Sigma_{H_{d}}$, unless we also ensure that there are no simultaneous solutions to $a_{5}=0$ and $\alpha_{4}=0$.

If one insists on extra $U(1)$ symmetries, then what are the possibilities for preventing dimension five proton decay? Embedding in $S O(10)$ does not really help because the $\mathbf{1 6}^{4}$ contains a singlet. One could try to hope embedding in $E_{6}$ (and breaking to $S U(5)_{\text {GUT }}$ by turning on a $U(1) \times U(1)$ bundle) will work, because the $\mathbf{2 7 ^ { 4 }}$ does not contain a singlet. It has been argued that the extra $U(1)$ symmetry is not sufficient to prevent all dimension five operators, which can still be generated through triplet exchange [81]. It seems that by further embedding in $E_{7}$ or $E_{8}$, sufficient $U(1)$ symmetries are available. We still need to explain how such symmetries are broken since they should not survive at low energies. We explained some options for breaking extra $U(1)$ 's in Section 5.2.

\footnotetext{
${ }^{13}$ It has recently been suggested that such a scenario for eliminating proton decay is ruled out due to subleading corrections to the localized contributions in the overlap integrals, so that we cannot make the couplings vanish exactly. This is only partially true. The localization of Yukawa couplings for massless chiral fields is an exact statement due to its nature as a product on Dolbeault cohomology [15]; there are no subleading corrections except for non-perturbative ones. On the other hand, the overlap with KK modes is not sharply localized as KK modes are not Dolbeault cohomology classes. Thus for dimension five proton decay we can only argue that it is suppressed. The superpotential of course is still exact, and we should understand this suppression as having to do with the Kähler potential.
} 
We would like to mention that an additional interesting possibility is to break the $U(1)$ symmetries through certain subleading corrections to the local model. To engineer this is we use a partially factorized "local" spectral cover, so as to get accidental $U(1)$ symmetries that forbid dimension four and/or dimension five proton decay in the local model, but embed it in a global model in which such symmetries are broken. The breaking of the $U(1)$ symmetries is then due to the subleading terms to the local ALE fibration, and for generic global UV completions of a local model we would expect such breaking to occur, because generic subleading terms would typically destroy any factorizability properties of the leading terms. Said differently, an exact extra $U(1)$ symmetry requires a global harmonic two-form with one index on the base and one on the fiber, but such forms are hard to come by as the elliptic fibration generically has $I_{1}$ singularities and the discriminant locus is irreducible away from the GUT brane. This is yet another manifestation of the principle that the fate of $U(1)$ symmetries is not determined locally but depends on the UV completion, as we saw earlier for $U(1)_{Y}$.

From the four-dimensional perspective this means that the $U(1)$ symmetries are only broken by operators suppressed by a high scale which is not native to the local model. There has been recent progress on defining a stable degeneration limit of global models in which a local model splits off from the rest of the Calabi-Yau [68]. One could use this technology to implement such an idea.

Another possible solution to the mu-problem and doublet/triplet splitting problem was recently described in [71]. The idea is to put the Higgs fields in the bulk of the 7-brane, rather than on a matter curve as discussed above. These models use a further $U(1)$ symmetry to eliminate dimension four proton decay.

Even if we suppressed the classical dimension four and five operators, they may still be generated non-perturbatively through $D 3$-instanton effects. Such effects are presumably too small to be observed.

The issue of dimension four and five proton decay has been much studied since this paper appeared, and we believe the last word has not been said on it.

\subsection{Operators of dimension six}

Assuming we have engineered the dimension four and five operators leading to proton decay to be sufficiently suppressed, we now turn to the dimension six operators (the four-fermi terms). These can be mediated by massive 
gauge bosons in the representation $(\mathbf{2}, \mathbf{3})_{-5 / 6}$. The internal wave functions of these particles are scalar fields supported in the bulk of the 7 -brane. ${ }^{14}$ We will follow the formulation of [18], making adjustments for the $F$-theory setting.

In four-dimensional $S U(5)$ GUTs, dimension six proton decay ${ }^{15}$ comes from exchange of massive gauge bosons in the $(\mathbf{2}, \mathbf{3})_{-5 / 6}$, resulting in the matrix element

$$
\mathscr{M} \sim g_{\mathrm{GUT}}^{2} \frac{J_{\mu} \tilde{J}^{\mu}(0)}{M^{2}}
$$

with $J^{\mu} \sim \bar{\Psi}_{\mathbf{1 0}} \gamma^{\mu} \Psi_{\mathbf{1 0}}+\bar{\Psi}_{\overline{\mathbf{5}}} \gamma^{\mu} \Psi_{\overline{\mathbf{5}}}$. In $F$-theory, there is a whole tower of massive gauge bosons with these quantum numbers. They are KK modes of the eight-dimensional $S U(5)$ gauge field, whose internal wave functions are eigen-functions of the scalar Laplacian on $S$ (the 7-brane worldvolume). Thus we can immediately write down the analogous expression:

$$
\mathscr{M} \sim g_{8}^{2} \int_{S \times S} d^{4} z_{1} d^{4} z_{2} \mathbf{j}^{\mu}\left(0, z_{1}\right) \tilde{\mathbf{j}}_{\mu}\left(0, z_{2}\right) G\left(z_{1}, z_{2}\right)
$$

Here the currents are constructed from the wave functions of the fermionic zero modes, $\mathbf{j}^{\mu}(x, z) \sim \bar{\Psi} \gamma^{\mu} \Psi(x, z)$, with $x$ denoting coordinates on $\mathbf{R}^{4}$ and $z$ denoting coordinates on $S$, and $G\left(z_{1}, z_{2}\right)$ is the Green's function for the scalar Laplacian on $S$, for scalar fields valued in the representation $(\mathbf{2}, \mathbf{3})_{-5 / 6}$. The fermionic zero modes are of the form

$$
\Psi(x, z)=\sum_{i} \chi_{i}(x) \otimes \psi_{i}(z)
$$

where $\chi_{i}$ is a four-dimensional spinor and $\psi_{i}(z)$ is an internal fermionic zero mode on the 7-brane worldvolume. The fermion zero modes, as discussed in detail in [15], correspond to harmonic $(0, q)$ forms on $S$. In order to extract the physical amplitudes, they must be properly normalized, i.e., $\int_{S} d^{4} z \psi_{i}^{\dagger} \psi_{i}=1$. Then we can pull out the four-dimensional part and write

\footnotetext{
${ }^{14}$ To be fair, as seen in Section 4.2 the internal wave functions for the longitudinal modes of these gauge bosons are exact one-forms on $S_{2}$. As we consider couplings to conserved currents, these modes are not relevant.

${ }^{15}$ In flipped $S U(5)$ or $S O(10)$ models there are additional dimension six operators mediated by heavy gauge bosons in the $(\mathbf{2}, \mathbf{3})_{-1 / 3}$ representation. Also, dimension six operators mediated by exchange of scalars in the $(\mathbf{1}, \overline{\mathbf{3}})_{1 / 3}$ are presumed absent due to whatever mechanism eliminated the dimensions five proton decay operators.
} 
the matrix element as ${ }^{16}$

$$
\mathscr{M} \sim g_{8}^{2} \sum_{i, j} J_{i, \mu} \tilde{J}_{j}^{\mu}(0) \int_{S \times S} d^{4} z_{1} d^{4} z_{2} \psi_{i}^{\dagger} \psi_{i}\left(z_{1}\right) \tilde{\psi}_{j}^{\dagger} \tilde{\psi}_{j}\left(z_{2}\right) G\left(z_{1}, z_{2}\right)
$$

where $J_{i}^{\mu}=\bar{\chi}_{i} \gamma^{\mu} \chi_{i}$. We can formally write the Green's function as

$$
G\left(z_{1}, z_{2}\right) \sim \sum_{k} \frac{\phi_{k}^{*}\left(z_{1}\right) \phi_{k}\left(z_{2}\right)}{M_{k}^{2}}
$$

where the $\phi_{k}(z)$ are the normalized eigenmodes of the Laplacian for $(\mathbf{2}, \mathbf{3})_{5 / 6^{-}}$ valued scalars. This representation makes the analogy with the fourdimensional expression (5.47) more clear.

If the integral is finite (i.e., if the sum over KK modes is convergent), then we will end up getting an expression of the same general form as (5.47), with $M$ replaced by the KK scale. Therefore we would like to investigate the UV behavior of the amplitude.

Let us first consider the $\mathbf{1 0}^{4}$ amplitude. Then both $\psi$ and $\tilde{\psi}$ are localized on $\Sigma_{\mathbf{1 0}}$, and both $z_{1}$ and $z_{2}$ are points on $\Sigma_{\mathbf{1 0}}$. The Green's function diverges as

$$
G\left(z_{1}, z_{2}\right) \sim \frac{1}{d\left(z_{1}, z_{2}\right)^{2}}
$$

as $z_{1} \rightarrow z_{2}$. Here $d\left(z_{1}, z_{2}\right)$ is the geodesic distance on $S$. In order to estimate the integral, we first change variables from $\left\{z_{1}, z_{2}\right\}$ to $\left\{z_{1}-z_{2}, z_{1}+z_{2}\right\}$. To make sense of this on a Riemannian manifold, we use the exponential map to set up a local coordinate system. This cannot be done globally, but for large $d$ the integral is effectively cut off and the only scale that can enter the large $d$ integration is $R_{\mathrm{KK}}$. Since we are interested in the UV behavior, we should concentrate on $z_{1}$ close to $z_{2}$. The change of variable introduces a Jacobian. To leading order for $z_{1}$ close to $z_{2}$, the Jacobian should not depend on the metric and we can use the flat space expressions. For our purposes we can also approximate the densities $\psi_{i}^{\dagger} \psi_{i}$ as constant of order $1 / R^{2}$ on the matter curve, and vanishing off the matter curve. Then the integral over $z_{1}+z_{2}$ gives the volume of $\Sigma_{\mathbf{1 0}}$ which is of order $R^{2}$. The

\footnotetext{
${ }^{16}$ We are being a little crude and use the label $i$ only to indicate the gauge indices; in principle there are also flavor indices.
} 
integral over $z_{1}-z_{2}$ gives

$$
\int_{S \times S} d^{4} z_{1} d^{4} z_{2} \psi_{i}^{\dagger} \psi_{i}\left(z_{1}\right) \tilde{\psi}_{j}^{\dagger} \tilde{\psi}_{j}\left(z_{2}\right) G\left(z_{1}, z_{2}\right) \sim \frac{1}{R^{2}} \int_{\epsilon} r d r \frac{1}{r^{2}}
$$

where $r=\left|z_{1}-z_{2}\right|$. The integral is cut-off at $r \sim R$ on the upper limit due to the fact that $\Sigma_{\mathbf{1 0}}$ is compact. However we are interested in the lower limit, and here it diverges logarithmically. To evaluate it, we need to go beyond the eight-dimensional field theory approximation. Any subleading corrections to our approximations though lead to integrals that are convergent as $r \rightarrow 0$, and can be evaluated without knowing anything about the UV completion.

The divergence indicates sensitivity to UV physics and so will be regularized by the non-perturbative completion of $F$-theory. Thus we expect that the divergence will be effectively cut off by including new modes with wave-lengths of order the ten-dimensional Planck length. Therefore we "regularize" the integral by cutting off the integration at $r \sim 1 / m_{10}$, where $m_{10}$ is the ten-dimensional Planck scale. Then our estimate for the size of the amplitude becomes

$$
\mathscr{M} \sim g_{8}^{2} M_{\mathrm{KK}}^{2} \log \left(M_{\mathrm{KK}} / m_{10}\right) J_{\mathbf{1 0}, \mu} \tilde{J}_{\mathbf{1 0}}^{\mu}(0)
$$

Even if we would have kept track of all the numerical factors and been able to evaluate the integrals, we do not know the exact coefficient due to lack of knowledge of the proper regularization of the Green's function. The dependence on the cut-off scale is very mild however. It is interesting to compare the parametric dependence of this expression with the analogous one for four-dimensional GUTs. Using our earlier expressions, $g_{8}^{2} M_{\mathrm{KK}}^{2} \sim$ $\alpha_{\mathrm{GUT}} / M_{\mathrm{KK}}^{2}$, so we can write this as ${ }^{17}$

$$
\mathscr{M} \sim \alpha_{\mathrm{GUT}} \log \left(\alpha_{\mathrm{GUT}}^{-1}\right) \frac{J_{\mathbf{1 0}, \mu} \tilde{J}_{\mathbf{1 0}}^{\mu}(0)}{M_{\mathrm{KK}}^{2}}
$$

There are two important qualitative differences here compared to the analogous expression for conventional four-dimensional GUTs. First, the amplitude is proportional to $M_{\mathrm{KK}}^{-2}$ rather than $M_{\mathrm{GUT}}^{-2}$. This is expected because the modes mediating this process are KK modes. Secondly, there is a logarithmic enhancement as $\alpha_{\mathrm{GUT}} \rightarrow 0$ compared to the expression for fourdimensional GUTs. This came about because the amplitude was divergent and sensitive to physics at the ten-dimensional Planck scale. In practice we have $\alpha_{\mathrm{GUT}}^{-1} \sim 25$ and the enhancement is not very large. Also it is probably

\footnotetext{
${ }^{17}$ This corrects an error in v1. The logarithmic dependence was pointed out in $[82,83]$.
} 
hard to get any universal results about the numerical values of the integrals. (According to a recent conjecture however, we can numerically approximate the Hermitian metric on the Higgs bundle [70]. If correct, it means that in principle we could systematically approximate the integrals, although we would still not know the correct value of the cut-off).

For the $\mathbf{1 0}^{2} \overline{\mathbf{5}}^{2}$ amplitude, we need to distinguish two cases. The easiest is if $\Sigma_{\mathbf{1 0}}$ and $\Sigma_{\overline{\mathbf{5}}}$ do not intersect, a possibility we considered above in order to suppress dimension four and dimension five baryon number violating operators. Then their separation depends on complex structure moduli but is at most order $R \sim V_{S}^{1 / 4}$ (scale of $S$ ), and the least we can get is something of the form

$$
\mathscr{M} \sim g_{8}^{2} V_{S}^{-1 / 2} J_{\mathbf{1 0}, \mu} \tilde{J}_{\overline{\mathbf{5}}}^{\mu}(0) \sim \alpha_{\mathrm{GUT}} \frac{J_{\mathbf{1 0}, \mu} \tilde{J}_{\overline{\mathbf{5}}}^{\mu}(0)}{M_{\mathrm{KK}}^{2}}
$$

Modulo the appearance of $M_{\mathrm{KK}}$ instead of $M_{\mathrm{GUT}}$, this has the same parametric dependence as the four-dimensional expression.

If we allow $\Sigma_{\mathbf{1 0}}$ and $\Sigma_{\overline{\mathbf{5}}}$ to come closer or if they intersect at a point, we need to be more careful. Although at the down type Yukawa intersection the curve $\Sigma_{\mathbf{5}}$ has a double point singularity, we will interpret this as two branches of distinct components of $\Sigma_{\boldsymbol{5}}$ intersecting there, one component supporting matter and the other supporting the Higgs. As we have seen, other intersections lead to dimension four proton decay. Then $\Sigma_{\overline{\mathbf{5}}_{m}}$ and $\Sigma_{\mathbf{1 0}}$ intersect each other transversely there, and we may take $\Sigma_{\mathbf{1 0}}$ to be defined by $z_{2}=0$ locally, and $\Sigma_{\overline{\mathbf{5}}_{m}}$ by $z_{2}=\alpha z_{1}$ for some constant $\alpha$.

Let us use $w_{1}$ as the local coordinate along $\Sigma_{\mathbf{1 0}}$ with $z_{1}\left(w_{1}\right)=w_{1}$, and $w_{2}$ be a local coordinate along $\Sigma_{\overline{\mathbf{5}}_{m}}$, such that $z_{1}\left(w_{2}\right)=w_{2}$ and $z_{2}\left(w_{2}\right)=\alpha w_{2}$. Let us further assume that the wave functions of the matter fields are nonvanishing at the intersection. This may not necessarily be the case, but the integrals only get more convergent when the wave-functions have such vanishing behavior, so we may restrict to the case when they do not vanish. Then we get the following integral:

$$
\begin{aligned}
& g_{8}^{2} \int d^{2} w_{1} d^{2} w_{2} \mathbf{j}_{\mathbf{1} \mathbf{0}}\left(w_{1}\right) G\left(w_{1}, w_{2}\right) \mathbf{j}_{\overline{\mathbf{5}}}\left(w_{2}\right) \\
& \quad \sim g_{8}^{2} R^{-4} \int d^{2} w_{1} d^{2} w_{2} \frac{1}{\left|w_{1}-w_{2}\right|^{2}+\left|\alpha w_{2}\right|^{2}} .
\end{aligned}
$$

Let us change variables from $\left(w_{1}, w_{2}\right)$ to $\left(w_{12}, w_{2}\right)$, where $w_{12}=w_{1}-w_{2}$. The Jacobian for this change of variable is simply the identity. Therefore 
the integral becomes

$$
g_{8}^{2} R^{-4} \int d^{2} w_{12} d^{2} w_{2} \frac{1}{\left|w_{12}\right|^{2}+\left|\alpha w_{2}\right|^{2}} .
$$

By a further change of variables we get

$$
g_{8}^{2} R^{-4}|\alpha|^{-2} \int_{\epsilon} d^{4} x \frac{1}{|x|^{2}} \sim g_{8}^{2} R^{-4}|\alpha|^{-2} \int_{\epsilon} r^{3} d r \frac{1}{r^{2}},
$$

which converges at short distances. Therefore this amplitude can be calculated in the effective eight-dimensional gauge theory, we do not need to know anything about the UV completion to compute this amplitude. Just as in (5.56) the parametric dependence is the same as conventional fourdimensional models (5.47), except with $M$ being $M_{\mathrm{KK}}$ rather than $M_{\mathrm{GUT}}$. The only exception could be if for some reason the intersection is not transverse and the angle $\alpha$ is zero, which could yield logarithmic behavior, but this is certainly not generic.

\subsection{Conclusions}

In this section, we investigated the issue of proton decay in $F$-theory GUTs. In generic models there is nothing to prevent fast proton decay, so there must be some extra structure. We discussed some options for eliminating significant proton decay through dimension four and five operators, and simultaneously solve the classical mu-problem, by factorizing the matter curves in certain ways and ensuring that we have the right flux through each irreducible component, or by using extra $U(1)$ symmetries which are broken by the subleading corrections to the local ALE fibration in a global model. Although it appears possible to satisfy experimental constraints, ideally one would like to connect this with other issues in flavor physics, and further research is desirable.

The dimension six operators receive contributions from massive KK gauge bosons. Their wavefunctions are supported in the bulk of the 7-brane, and so such contributions can likely not be suppressed. We have found two qualitative differences with conventional four-dimensional models: there is a parametric difference between the $p \rightarrow \pi^{0} e_{L}^{+}$and $p \rightarrow \pi^{0} e_{R}^{+}$, and further the scale differs parametrically from the GUT scale. Although there is uncertainly about the numerical prefactors, these qualitative differences are universal and could potentially serve as smoking guns for $F$-theoretic GUT models. 
Processes coming from the 10 $\mathbf{1 0}$ OPE may be enhanced with respect to those coming from the $\mathbf{1 0} \cdot \overline{\mathbf{5}} \mathrm{OPE}$, due to a parametric enhancement of dimension six proton decay by a factor $\log \left(\alpha_{\mathrm{GUT}}^{-1}\right)$. Whether this would translate to an actual enhancement in the real world also depends on the numerical factors, which are hard to calculate, but at least it constitutes a qualitative difference with four-dimensional models. Such a qualitative difference is not uncommon in Kaluza-Klein models, but the precise form depends on the dimensionality: in $M$-theory/IIA models of unification, an $\alpha_{\mathrm{GUT}}^{-1 / 3}$ enhancement in the $\mathbf{1 0} \cdot \mathbf{1 0} \mathrm{OPE}$ was found compared to fourdimensional GUTs $[18,19]$.

The second effect comes from the KK scale being lowered compared to the GUT scale, which is parametric when the GUT group is broken by $U(1)_{Y}$ flux. The origin of this effect was explained in Section 4. If indeed the KK scale is lowered even slightly, we get a rather large enhancement of proton decay since $|\mathscr{M}|^{-2}$ scales as $M_{\mathrm{KK}}^{4}$. This is a surprising difference with conventional four-dimensional models, where the dimension 6 operators are mediated by $X$ and $Y$ bosons which have masses of order $M_{\mathrm{GUT}}$. This situation is also different from the $M$-theory unification models; in [18] it was found that after taking into account one-loop threshold corrections in $M$-theory, there is no parametric separation between $M_{\mathrm{KK}}$ and $M_{\mathrm{GUT}}$.

The proton life-time is of the form

$$
\tau_{p} \sim|\mathscr{M}|^{-2} m_{p}^{-5}
$$

In four-dimensional GUTs, with $M_{\mathrm{GUT}} \sim 3 \times 10^{16} \mathrm{GeV}$, we get [74] $\tau(p \rightarrow$ $\left.\pi^{0} \mathrm{e}^{+}\right) \sim 10^{35 \pm 1} \mathrm{yrs}$. This is out of range for current experiments, which provide a bound $\tau\left(p \rightarrow \pi^{0} \mathrm{e}^{+}\right)>5 \times 10^{33} \mathrm{yrs}$. The numerical pre-factor in $F$-theory models depends on the details of the geometry of the configuration, so it seems hard to make a universal statement. If, hypothetically, the numerical factors are similar to those of four-dimensional models, then with $\alpha_{\mathrm{GUT}} \sim 1 / 25$ the lifetime for $p \rightarrow \pi^{0} e_{L}^{+}$could get reduced by a factor of 10 compared to $p \rightarrow \pi^{0} e_{R}^{+}$due to the first effect. Due to the second effect, for $\Lambda \sim R_{B_{3}} m_{10}^{2}$ the lifetime through dimension six decays may be brought down by a factor of $10^{1.5}$, bringing it to the verge of detection.

\section{Acknowledgments}

MW would like to thank K. Köhler for correspondence on Ray-Singer torsion; J. Conlon for discussions on the unification scale in string models; and I. Adam, Y. Oz and S. Theisen for discussion related to this project. MW 
would also like to thank UPenn, LPTHE Jussieu, CUHK, HK University, CERN, Cambridge University, and the Simons workshop at SUNYSB for hospitality while this work was in progress and the opportunity to present some of these results. The research of MW was supported in part by a Curie fellowship under contract number MRTN-CT-2004-512294. R.D. is partially supported by NSF grant no. DMS 0612992 and NSF Research and Training Grant no. DMS 0636606.

\section{Appendix A GUT breaking fluxes constructed through the cylinder map}

In this appendix we would like to give a more detailed discussion of quantization constraints on GUT breaking fluxes in heterotic and local $F$-theory models. We will see how to construct models without exotic matter, and we will learn that this requires the Noether-Lefschetz fluxes of [22].

We consider an elliptically fibered Calabi-Yau three-fold $Z$ with a $U(5) \times$ $U(1)$ bundle $V \oplus K^{-1}$. We require that $\operatorname{det} V=K$ so that the structure group embeds in $S U(6) \subset E_{8}$. In other words, we consider a rank six bundle with structure group $U(5)$. We further assume our bundle $V$ can be constructed using spectral covers. Such bundles sometimes have the larger structure group $S U(5) \times U(1)$ rather than $U(5)=S U(5) \times Z_{5} U(1)$, which can cause trouble with exotic matter as we will see below. Then the spectral data consist of a degree six spectral cover and a rank one spectral sheaf on it. The six sheets may be labelled by the following roots of $E_{8}$ :

$$
\begin{array}{ll}
\sigma_{1}=\alpha_{5}, & \sigma_{4}=\alpha_{5}+\alpha_{4}+\alpha_{3}+\alpha_{2}, \\
\sigma_{2}=\alpha_{5}+\alpha_{4}, & \sigma_{5}=\alpha_{5}+\alpha_{4}+\alpha_{3}+\alpha_{2}+\alpha_{1}, \\
\sigma_{3}=\alpha_{5}+\alpha_{4}+\alpha_{3}, & \sigma_{6}=\alpha_{5}+\alpha_{4}+\alpha_{3}+\alpha_{2}+\alpha_{1}+\alpha_{-\theta} .
\end{array}
$$

The degree six spectral cover decomposes into an irreducible five-fold spectral cover $\pi_{C_{5}}: C_{5} \rightarrow S$ with sheets $\left\{\sigma_{2}, \ldots, \sigma_{6}\right\}$ and a degree one piece which is just $\sigma_{1}=\sigma_{B_{2}}$, so $C_{6}=C_{5} \cup \sigma_{1}$. Since we allow for a $U(5)$ structure group, we have a wider set of choices for the spectral line bundle $L_{5}$ on $C_{5}$ :

$$
c_{1}\left(L_{5}\right)=-\frac{1}{2} c_{1}(C)+\frac{1}{2} \pi_{C_{5}}^{*} c_{1}\left(B_{2}\right)+\gamma,
$$

with $\Sigma=C \cap \sigma_{B_{2}}$. For $S U(5)$ bundles, one would also require that $\pi_{C_{5} *} \gamma=$ 0 . Here instead we turn on a line bundle $\zeta^{-1}$ on $\sigma_{1}$ so that

$$
c_{1}(\zeta)=c_{1}\left(\pi_{C_{5} *} \gamma\right)=c_{1}\left(\left.\operatorname{det}(V)\right|_{B_{2}}\right) \in H^{2}\left(B_{2}\right) .
$$


In fact, this is still not the most general construction; we may further twist $V$ by a line bundle $Q$ with $c_{1}(Q)=q p_{Z}^{*} \Sigma$. Then we find [33]

$$
c_{1}(V \otimes Q)=n q p_{Z}^{*} \Sigma+p_{Z}^{*} c_{1}(\zeta),
$$

which determines the line bundle $K$ we have to turn on to compensate. Turning on $q \neq 0$ corresponds to making $\sigma_{1}$ different form the zero section $\sigma_{B_{2}}$, i.e., changing the four-fold rather than turning on $G$-flux. In terms of the eight-dimensional gauge theory, it corresponds to breaking the gauge group by turning on a non-zero profile for an abelian Higgs field. For more discussion of the latter as a mechanism for breaking the GUT group, see [22]. In the following however we keep $q=0$.

In order to break the GUT group without massless lepto-quarks, we want $c_{1}(\zeta)$ to be a primitive class with $c_{1}(\zeta) \cdot c_{1}\left(B_{2}\right)=0, c_{1}(\zeta) \cdot t=0$ and $c_{1}(\zeta)^{2}=$ -2 . Furthermore, for $L_{5}$ to exist the first Chern class of $L_{5}$ must be an integer class on $C_{5}$. As a simple Ansatz, we could try to take $\gamma$ of the form

$$
\gamma=\lambda \gamma_{u}+\frac{1}{5} \pi_{C_{5}}^{*} c_{1}(\zeta)
$$

where $\pi_{C_{5} *} \gamma_{u}=0$. Now we claim that if $\gamma_{u}$ is given by the universal flux:

$$
\gamma_{u}=5\left[\Sigma_{\mathbf{1 0}}\right]-p^{*}\left(\eta-5 c_{1}\right)
$$

then these conditions are not compatible, as we now show. First of all, note that any non-integrality in $\frac{1}{5} \pi_{C_{5}}^{*} c_{1}(\zeta)$ cannot be cancelled by $\frac{1}{2} r+\lambda \gamma_{u}$ unless $c_{1}(\zeta)$ is a multiple of $\eta-5 c_{1}$. But this is impossible on a del Pezzo surface as $\eta-5 c_{1}$ is effective, hence $c_{1} \cdot\left(\eta-5 c_{1}\right)>0$, but we had $c_{1}\left(B_{2}\right) \cdot c_{1}(\zeta)=0$. (One may strengthen this and also rule out other cases like $d P_{9}$ ). Therefore $p^{*} c_{1}(\zeta)$ should be divisible by 5 . But then $p^{*} c_{1}(\zeta)^{2}$ must be divisible by 25 . However,

$$
\pi_{C_{5}}^{*} c_{1}(\zeta) \cdot \pi_{C_{5}}^{*} c_{1}(\zeta)=c_{1}(\zeta) \cdot \pi_{C_{5} *} \pi_{C_{5}}^{*} c_{1}(\zeta)=-10
$$

which does not contain any squares, so $\pi_{C_{5}}^{*} c_{1}(\zeta)$ is in fact primitive. We conclude that there is a problem with exotic matter for GUT breaking by $U(1)$ fluxes in the heterotic string, if we only use this special flux. This is the heterotic version of the puzzle we discussed in Section 2.2: the $U(5)$ bundle cannot be the product of an $S U(5)$ bundle and a $U(1)$ bundle if we want to avoid exotic matter. In particular, previously proposed models along these lines turn out to have exotic matter. 
We will now show that exotic matter can be avoided if we use more general fluxes, namely the Noether-Lefschetz fluxes discovered in [22]. Consider a flux of the form

$$
c_{1}\left(L_{5}\right)=\frac{1}{2} r+\lambda \gamma_{u}+\alpha=\frac{1}{2} r+\tilde{\gamma}+\frac{1}{5} \pi_{C_{5}}^{*} c_{1}(\zeta),
$$

where $c_{1}(\zeta)=\pi_{C_{5} *} \alpha, r=-c_{1}\left(C_{5}\right)+\pi_{C_{5}}^{*} c_{1}\left(B_{2}\right)$. In the last equality, we have artificially split the flux into a flux $\tilde{\gamma}$ that commutes with $S U(5)_{\mathrm{GUT}}$ :

$$
\pi_{C_{5} *} \tilde{\gamma}=\lambda \pi_{C_{5} *} \gamma_{u}+\pi_{C_{5} *}\left(\alpha-\frac{1}{5} \pi_{C_{5}}^{*} \pi_{C_{5} *} \alpha\right)=0
$$

and a flux $\frac{1}{5} \pi_{C_{5}}^{*} c_{1}(\zeta)$ that is responsible for breaking $S U(5)_{\mathrm{GUT}}$ to the Standard Model. However $\frac{1}{5} \pi_{C_{5}}^{*} c_{1}(\zeta)$ is generally not an integer class. So we cannot simply turn off the GUT breaking flux if we are to satisfy the quantization constraints. This would result in an inconsistent model.

With the above definitions, $c_{1}\left(L_{5}\right)$ is naturally an integral class when $\lambda-\frac{1}{2}$ is an integer and $\alpha$ is an integral class. So we only need to construct a $(1,1)$ class $\alpha$ such that $\pi_{C_{5} *} \alpha \cdot c_{1}=0, \pi_{C_{5} *} \alpha \cdot t=0$ and $\left(\pi_{C_{5} *} \alpha\right)^{2}=-2$. This can certainly be done, for instance as in [22], but it requires that we adjust/stabilize some of the moduli. Otherwise the only available fluxes are the class of the matter curve and pull-backs of classes in $B_{2}$, which leads to the universal flux (A.6) and thus to exotic matter. Hence we conclude that absence of light scalar and vector lepto-quarks is perfectly consistent with the quantization conditions, provided we go slightly outside of the usual framework of (A.5) and make use of all available fluxes.

If we use the construction in [22] then we have $\int_{\Sigma_{\mathbf{1 0}}} \alpha=0$ and turning on $\alpha$ does not change the net chirality. So we immediately get some toy models with three generations and no exotic matter by using the explicit examples in Section 4 of [15] and turning on $\alpha$. The main downside of these toy models is that for generic complex structure moduli, they have no light Higgses (i.e., the model suffers from the $\mu$-problem), and if we would tune to get such Higgses, the model would typically suffer from fast proton decay and the Yukawa couplings would not be hierarchical. Some extra structure is needed to explain the mu-problem, the stability of the proton and flavor.

Let us be a little more explicit about the resulting $G$-fluxes in $F$-theory. Again we focus on the case of $S U(5)_{\text {GUT }}$ models broken to the Standard Model by hypercharge flux. Then this is a special case of an $S l(6)$ cover, which splits as $5+1$. All the other components of the $E_{8}$ cover can be expressed in terms of this fundamental cover. We use the following notation, 
similar to Appendix C of [15]:

$$
\begin{array}{rlrl}
\pi: Y_{4} & \rightarrow B_{3} & & \text { elliptic fibration } \\
\sigma_{B_{3}}: B_{3} \rightarrow Y_{4} & \text { the section } \\
\rho: B_{3} \rightarrow B_{2} & P^{1} \text { fibration } \\
Z \subset Y_{4} & \pi^{-1} \text { of a section of } \rho . \\
p: Y_{4} \rightarrow B_{2} & d P_{9} \text { fibration. } \\
\pi_{C}: C_{6} \rightarrow B_{2} & \text { the heterotic spectral cover } \\
p_{R}: R \rightarrow C_{6} & \text { the "cylinder", or union of lines in the } d P_{8}^{\prime} \text { s } \\
& \text { (i.e. sections of } \left.d P_{9}^{\prime} \text { s, disjoint from } \sigma\right) \\
j:\left(C_{6}=R \cap Z\right) \subset R & \text { parametrized by points of } C . \\
i: R \hookrightarrow Y & \text { the inclusion "at infinity" } \\
\operatorname{Pr}: H^{i}\left(Y_{4}\right) \rightarrow H^{i}\left(Y_{4}\right) & \text { orthogonal projection on }\left[\pi^{*} H^{i}\left(B_{3}\right)\right]^{\perp}
\end{array}
$$

We further consider the split $C_{6}=C_{5} \cup C_{1}$, where $C_{1}$ coincides with the zero section $B_{2}$. In $F$-theory, the $G$-flux dual to the bundle $V \oplus \operatorname{det}(V)^{-1}$ is then

$$
\begin{aligned}
\mathrm{G} & =\operatorname{Pr}\left[i_{R_{5} *} p_{R_{5}}^{*} \gamma-i_{R_{1} *} p_{R_{1}}^{*} c_{1}(\zeta)\right] \\
& =i_{R_{5} *} p_{R_{5}}^{*} \gamma-i_{R_{1} *} p_{R_{1}}^{*} c_{1}(\zeta)-n_{\gamma}\left[d P_{9}\right]
\end{aligned}
$$

Here we used $\gamma=c_{1}\left(L_{5}\right)-\frac{1}{2} r$ to represent the flux of $L_{5}$. We recall that $c_{1}(\zeta)=-\pi_{C_{5} *} \gamma$. We compute that

$$
n_{\gamma}=\gamma \cdot C_{5} \Sigma-c_{1}(\zeta) \cdot B_{2} c_{1}\left(K_{B_{2}}\right)
$$

Since we are assuming that $c_{1}(\zeta)$ is orthogonal to $K_{S}$, the last part simply drops out. The map $p_{R}$ associates lines in $d P_{8}$ (or sections of $d P_{9}$ ) with points on $T^{2}$ and is defined using the embedding $S U(6) \in E_{8}$ discussed in equation (A.1). Note that in the notation of Section 2.2, essentially we have a fractional line bundle $L^{1 / 6}=\zeta^{1 / 5}$ on each sheet of the irreducible five-fold cover $C_{5}$, and $L^{-5 / 6}=\zeta^{-1}$ on the sixth sheet.

It remains to say something about the $D$-terms. There are two cases to be addressed. First, it is possible that our abelian gauge symmetry does not couple to RR axions for topological reasons, as we imposed for hypercharge. In this case, by SUSY there is also no corresponding Fayet-Iliopoulos term. Such configurations are naturally polystable, with slope zero. 
Now suppose that we do have such a coupling. We take a general Kähler class of the form

$$
J=t_{1} p^{*} J_{B_{2}}+t_{2} J_{\infty}
$$

where $J_{B_{2}}$ is a class in $H^{2}\left(B_{2}\right)$, and $J_{\infty}$ is the Poincaré dual of $B_{2}$ in $B_{3}$ (where we use the embedding at infinity here - this differs from the embedding at the location of the singular locus only by a class in $H^{2}\left(B_{2}\right)$ ). we need both $t_{1}$ and $t_{2}$ large in Planck units. The small angle limit, where we can trust the $8 d$ gauge theory description, corresponds to $t_{1} \gg t_{2}$. We are interested in the Fayet-Iliopoulos term

$$
\xi \sim m_{10}^{4} \int_{Y_{4}} G \wedge J \wedge \omega^{Y} .
$$

The intersection of $G$ with $J_{\infty}$ would generally be non-zero, but in the present case we know that $\omega_{Y}$ is supported at the zero section (see equation $(2.21)$ ), whereas $J_{\infty}$ is localized at the infinity section, and hence the intersection vanishes. The Fayet-Iliopoulos parameter then only depends only on the intersection of $G$ with $p^{*} J_{B_{2}}$, which is proportional to $J_{B_{2}} \cdot B_{2} c_{1}(\zeta)$. In particular, it vanishes if $J_{B_{2}} \cdot c_{1}(\zeta)=0$. For more general $U(1)$ 's, the FayetIliopoulos parameter can depend on the extension to the global model.

\section{Appendix B Metric anomaly for holomorphic torsion}

Let $X$ denote a complex manifold of dimension $d=2 n$ with metric $g$, and let $V$ be a holomorphic bundle on $X$ with Hermitean metric $h$. Let $\mathbf{T}(X, V)$ denote the Ray-singer torsion for $V$. The torsion is not invariant under general changes of the metrics $g$ and $h$. Consider a one parameter family of metrics $g_{t}, h_{t}$. The variational formula of [51] is as follows:

$$
\begin{aligned}
\frac{1}{2 \pi} \frac{\partial}{\partial t} \mathbf{T}(X, V)= & \frac{1}{2 \pi} \frac{1}{2} \sum(-1)^{q} \operatorname{Tr}\left(\alpha_{t} P_{0, q}^{t}\right)-\left.\frac{1}{2} \int_{X} \frac{\partial}{\partial s}\right|_{s=0} \\
& \times\left[\mathbf{T} \mathbf{d} \frac{1}{2 \pi}\left(i R+s g^{-1} \partial_{t} g\right) \mathbf{c h} \frac{1}{2 \pi}\left(i F+s h^{-1} \partial_{t} h\right)\right]_{n+1}
\end{aligned}
$$

Here $\alpha_{t}=*_{t}^{-1} \partial_{t} *_{t}$, the operator $P_{0, q}^{t}$ projects on the zero modes of the indicated degree, and the subscript $n+1$ on the second line means that one should take the piece of the expression of degree $n+1$.

In order to get the scale dependence, we consider rescaling the metric as $g_{\mu \nu} \rightarrow t g_{\mu \nu}$. That is, we only need the following special case: $h^{-1} \partial h=$ 
$0, g^{-1} \partial g=\frac{1}{t} I_{2 n}(1 / t$ times the $2 n \times 2 n$ identity matrix $)$. We claim that

$$
{ }_{t} \omega_{0, q} \propto t^{d / 2-q}
$$

Here $t^{d / 2}$ arises from the factor of $\sqrt{g}$ in $*$, and $t^{-q}$ arises because we need $q$ factors of $g^{\mu \nu}$ to contract the indices of $\omega_{0, q}$ with the $\epsilon$ tensor. Therefore

$$
\begin{aligned}
\sum(-1)^{q} \operatorname{Tr}\left(\alpha_{t} P_{0, q}^{t}\right) & =\frac{1}{t} \sum(-1)^{q} h^{q}(d / 2-q) \\
& =\frac{1}{t}\left(\frac{d}{2} \chi(V)+\sum(-1)^{q+1} q h^{q}\right),
\end{aligned}
$$

where $h^{q}$ is the number of zero modes in $\Omega^{0, q}(V)$.

For our purposes we need two special cases. We use the splitting principle in order to simplify the calculation. Recall that

$$
T d(x)=\frac{x}{1-\mathrm{e}^{-x}}=1+\frac{1}{2} x+\frac{1}{12} x^{2}-\frac{1}{720} x^{4}+\cdots .
$$

We also implicitly assume that $V$ has rank one. The calculation is easily adjusted for higher rank.

For a curve:

$$
\begin{aligned}
& \left.\frac{\partial}{\partial s}\right|_{s=0}[\mathbf{T d}(T) \mathbf{c h}(V)]_{2} \\
& \quad=\left.\frac{1}{2 \pi t} \frac{\partial}{\partial s}\right|_{s=0}\left[\left(1+\frac{1}{2}(x+s)+\frac{1}{12}(x+s)^{2}\right)\left(1+c_{1}+c h_{2}\right)\right]_{2} \\
& \quad=\left.\frac{1}{2 \pi t} \frac{\partial}{\partial s}\right|_{s=0}\left[c h_{2}+\frac{1}{2}(x+s) c_{1}+\frac{1}{12}(x+s)^{2}\right] \\
& \quad=\frac{1}{2 \pi t}\left(\frac{1}{2} c_{1}(V)+\frac{1}{6} c_{1}(T)\right) .
\end{aligned}
$$

In the above equation, in order to avoid having to keep writing $s / 2 \pi t$, we redefined $s \rightarrow 2 \pi t s$ which of course gives the same result. Combining with (B.3), we get

$$
2 \mathbf{T}(X, V) \sim\left[\left(h^{0}(X, V)-\int_{X}\left(\frac{1}{2} c_{1}(V)+\frac{1}{6} c_{1}(T)\right)\right] \log t .\right.
$$

One may check this expression is invariant under Serre duality, $\mathbf{T}(X, V)=$ $\mathbf{T}\left(X, K \otimes V^{*}\right)$. 
For a surface:

$$
\begin{aligned}
& \left.\frac{\partial}{\partial s}\right|_{s=0}[\mathbf{T d}(T) \mathbf{c h}(V)]_{3} \\
& =\left.\frac{1}{2 \pi t} \frac{\partial}{\partial s}\right|_{s=0}\left[\prod_{i=1,2}\left(\left(1+\frac{1}{2}\left(x_{i}+s\right)+\frac{1}{12}\left(x_{i}+s\right)^{2}\right) \sum_{j} c h_{j}(V)\right]_{3}\right. \\
& =\frac{1}{2 \pi t}\left(c h_{2}(V)+\frac{5}{12} c_{1}(V) c_{1}(T)+\frac{1}{24} c_{1}(T)^{2}+\frac{1}{12} c_{2}(T)\right) .
\end{aligned}
$$

Combining with (B.3), we get

$$
\begin{aligned}
2 \mathbf{T}(X, V) \sim & {\left[\sum(-1)^{q}(2-q) h^{q}(X, V)\right.} \\
& \left.-\int_{X}\left(c h_{2}(V)+\frac{5}{12} c_{1}(V) c_{1}(T)+\frac{1}{24} c_{1}(T)^{2}+\frac{1}{12} c_{2}(T)\right)\right] \log t .
\end{aligned}
$$

Again one may check this expression behaves appropriately under Serre duality, in this case $\mathbf{T}(X, V)=-\mathbf{T}\left(X, K \otimes V^{*}\right)$. This is a useful check on some of the coefficients.

\section{Appendix C Roots of $E_{8}$}

In this brief section, we would like to explicitly write out the roots of $E_{8}$ and their decomposition under the $S U(5)_{H} \times S U(5)_{\text {GUT }}$ subgroup of $E_{8}$. We use the labelling of the $E_{8}$ Dynkin diagram shown earlier in this paper, and define $\alpha_{-\theta}$ to be the negative of the highest root:

$$
\alpha_{-\theta}=-2 \alpha_{1}-3 \alpha_{2}-4 \alpha_{3}-5 \alpha_{4}-6 \alpha_{5}-4 \alpha_{6}-2 \alpha_{7}-3 \alpha_{8} .
$$

The adjoint representation is 248-dimensional, consisting of eight Cartan generators, 120 positive and 120 negative roots. To make the table less cluttered, will list only 120 of the roots; the remaining roots are the negative of those that are written. For the case of the $(\mathbf{1}, \mathbf{2 4})$ and $(\mathbf{2 4}, \mathbf{1})$ representations, we further have to add the Cartan generators in order to recover the full representation. The roots are divided up as follows: 
$(1,24)$ :

$$
\left\{\begin{array}{c}
\alpha_{5}, \alpha_{6}, \alpha_{7}, \alpha_{8} \\
\alpha_{5}+\alpha_{6}, \alpha_{6}+\alpha_{7}, \alpha_{5}+\alpha_{8}, \alpha_{5}+\alpha_{6}+\alpha_{8} \\
\alpha_{5}+\alpha_{6}+\alpha_{7}, \alpha_{5}+\alpha_{6}+\alpha_{7}+\alpha_{8}
\end{array}\right\}
$$

$(24,1)$ :

$$
\left\{\begin{array}{c}
\alpha_{-\theta}, \alpha_{1}, \alpha_{2}, \alpha_{3}, \\
\alpha_{-\theta}+\alpha_{1}, \alpha_{1}+\alpha_{2}, \alpha_{2}+\alpha_{3}, \\
\alpha_{-\theta}+\alpha_{1}+\alpha_{2}, \\
\alpha_{1}+\alpha_{2}+\alpha_{3} \\
\alpha_{-\theta}+\alpha_{1}+\alpha_{2}+\alpha_{3} .
\end{array}\right\}
$$

$(5,10):$

$$
\left\{\begin{array}{c}
\alpha_{4} \\
\alpha_{3}+\alpha_{4} \\
\alpha_{2}+\alpha_{3}+\alpha_{4} \\
\alpha_{1}+\alpha_{2}+\alpha_{3}+\alpha_{4} \\
\alpha_{-\theta}+\alpha_{1}+\alpha_{2}+\alpha_{3}+\alpha_{4}
\end{array}\right\}+\left\{\begin{array}{c}
\alpha_{5} \\
\alpha_{5}+\alpha_{6} \\
\alpha_{5}+\alpha_{8} \\
\alpha_{5}+\alpha_{6}+\alpha_{7} \\
\alpha_{5}+\alpha_{6}+\alpha_{8} \\
\alpha_{5}+\alpha_{6}+\alpha_{7}+\alpha_{8} \\
2 \alpha_{5}+\alpha_{6}+\alpha_{8} \\
2 \alpha_{5}+\alpha_{6}+\alpha_{7}+\alpha_{8} \\
2 \alpha_{5}+2 \alpha_{6}+\alpha_{7}+\alpha_{8}
\end{array}\right\}
$$

$(10, \overline{5})$ :

$$
\left\{\begin{array}{c}
\alpha_{3}+2 \alpha_{4} \\
\alpha_{2}+\alpha_{3}+2 \alpha_{4} \\
\alpha_{2}+2 \alpha_{3}+2 \alpha_{4} \\
\alpha_{1}+\alpha_{2}+\alpha_{3}+2 \alpha_{4} \\
\alpha_{1}+\alpha_{2}+2 \alpha_{3}+2 \alpha_{4} \\
\alpha_{1}+2 \alpha_{2}+2 \alpha_{3}+2 \alpha_{4} \\
\alpha_{-\theta}+\alpha_{1}+\alpha_{2}+\alpha_{3}+2 \alpha_{4} \\
\alpha_{-\theta}+\alpha_{1}+\alpha_{2}+2 \alpha_{3}+2 \alpha_{4} \\
\alpha_{-\theta}+\alpha_{1}+2 \alpha_{2}+2 \alpha_{3}+2 \alpha_{4} \\
\alpha_{-\theta}+2 \alpha_{1}+2 \alpha_{2}+2 \alpha_{3}+2 \alpha_{4}
\end{array}\right\}+\left\{\begin{array}{c}
2 \alpha_{5}+\alpha_{6}+\alpha_{8} \\
2 \alpha_{5}+\alpha_{6}+\alpha_{7}+\alpha_{8} \\
2 \alpha_{5}+2 \alpha_{6}+\alpha_{7}+\alpha_{8} \\
3 \alpha_{5}+2 \alpha_{6}+\alpha_{7}+\alpha_{8} \\
3 \alpha_{5}+2 \alpha_{6}+\alpha_{7}+2 \alpha_{8}
\end{array}\right\}
$$




\section{References}

[1] H. Georgi, H.R. Quinn and S. Weinberg, Hierarchy of interactions in unified gauge theories, Phys. Rev. Lett. 33 (1974), 451.

[2] S. Dimopoulos, S. Raby and F. Wilczek, Supersymmetry and the scale of unification, Phys. Rev. D 24 (1981), 1681.

[3] H. Georgi and S.L. Glashow, Unity of all elementary particle forces, Phys. Rev. Lett. 32 (1974), 438.

[4] Y. Hosotani, Dynamical mass generation by compact extra dimensions, Phys. Lett. B 126 (1983), 309.

[5] E. Witten, Symmetry breaking patterns in superstring models, Nucl. Phys. B 258 (1985), 75.

[6] P. Candelas, G.T. Horowitz, A. Strominger and E. Witten, Vacuum configurations for superstrings, Nucl. Phys. B 258 (1985), 46.

[7] I. Antoniadis, E. Kiritsis and T.N. Tomaras, A D-brane alternative to unification, Phys. Lett. B 486 (2000), 186, [arXiv:hep-ph/0004214].

[8] G. Aldazabal, L.E. Ibanez, F. Quevedo and A.M. Uranga, Dbranes at singularities: a bottom-up approach to the string embedding of the standard model, J. High Energy Phys. 08 (2000), 002, [arXiv:hep-th/0005067].

[9] D. Berenstein, V. Jejjala and R.G. Leigh, The standard model on a Dbrane, Phys. Rev. Lett. 88 (2002), 071602, [arXiv:hep-ph/0105042].

[10] H. Verlinde and M. Wijnholt, Building the standard model on a D3brane, J. High Energy Phys. 01 (2007), 106, [arXiv: hep-th/0508089].

[11] E. Witten, Strong coupling expansion of Calabi-Yau compactification, Nucl. Phys. B 471 (1996), 135, [arXiv:hep-th/9602070].

[12] T. Pantev and M. Wijnholt, Hitchin's equations and M-theory phenomenology, arXiv:0905.1968 [hep-th].

[13] M. Bershadsky, K.A. Intriligator, S. Kachru, D.R. Morrison, V. Sadov and C. Vafa, Geometric singularities and enhanced gauge symmetries, Nucl. Phys. B 481 (1996), 215, [arXiv:hep-th/9605200].

[14] R. Friedman, J. Morgan and E. Witten, Vector bundles and F theory, Commun. Math. Phys. 187 (1997), 679, [arXiv:hep-th/9701162].

[15] R. Donagi and M. Wijnholt, Model building with F-theory, arXiv:0802 . 2969 [hep-th].

[16] C. Beasley, J.J. Heckman and C. Vafa, GUTs and exceptional branes in F-theory - I, arXiv:0802.3391 [hep-th].

[17] H. Hayashi, R. Tatar, Y. Toda, T. Watari and M. Yamazaki, New Aspects of Heterotic-F Theory Duality, arXiv:0805.1057 [hep-th]. 
[18] T. Friedmann and E. Witten, Unification scale, proton decay, and manifolds of G(2) holonomy, Adv. Theor. Math. Phys. 7 (2003), 577, [arXiv:hep-th/0211269].

[19] I.R. Klebanov and E. Witten, Proton decay in intersecting Dbrane models, Nucl. Phys. B 664 (2003), 3, [arXiv:hep-th/ 0304079].

[20] C. Beasley, J.J. Heckman and C. Vafa, GUTs and Exceptional branes in F-theory - type II: experimental predictions, arXiv:0806.0102 [hep-th].

[21] R. Tatar and T. Watari, GUT relations from string theory compactifications, arXiv:0806.0634 [hep-th].

[22] R. Donagi and M. Wijnholt, Higgs bundles and UV completion in F-Theory, arXiv:0904.1218 [hep-th].

[23] R. Donagi and M. Wijnholt, $M S W$ instantons, arXiv:1005.5391 [hep-th].

[24] R. Blumenhagen, A. Collinucci and B. Jurke, On Instanton effects in F-theory, J. High Energy Phys. 08 (2010), 079, [arXiv:1002.1894 [hep-th]].

[25] L.J. Hall and Y. Nomura, Grand unification in higher dimensions, Annals Phys. 306 (2003), 132, [arXiv:hep-ph/0212134].

[26] K.R. Dienes, E. Dudas and T. Gherghetta, Grand unification at intermediate mass scales through extra dimensions, Nucl. Phys. B 537 (1999), 47, [arXiv:hep-ph/9806292].

[27] E. Witten, New issues in manifolds of SU(3) holonomy, Nucl. Phys. B 268 (1986), 79.

[28] M. Buican, D. Malyshev, D.R. Morrison, H. Verlinde and M. Wijnholt, D-branes at singularities, compactification, and hypercharge, J. High Energy Phys. 01 (2007), 107, [arXiv:hep-th/0610007].

[29] M. Wijnholt, Geometry of particle physics, Adv. Theor. Math. Phys. 13(4) (2009), 947-990, arXiv:hep-th/0703047.

[30] B. Andreas and D. Hernandez Ruiperez, $U(n)$ vector bundles on CalabiYau threefolds for string theory compactifications, Adv. Theor. Math. Phys. 9 (2005), 253, [arXiv:hep-th/0410170].

[31] R. Blumenhagen, G. Honecker and T. Weigand, Loop-corrected compactifications of the heterotic string with line bundles, J. High Energy Phys. 06 (2005), 020, [arXiv:hep-th/0504232].

[32] R. Blumenhagen, S. Moster and T. Weigand, Heterotic GUT and standard model vacua from simply connected Calabi-Yau manifolds, Nucl. Phys. B 751 (2006), 186, [arXiv:hep-th/0603015]. 
[33] R. Blumenhagen, S. Moster, R. Reinbacher and T. Weigand, Massless spectra of three generation $U(N)$ heterotic string vacua, J. High Energy Phys. 05 (2007), 041, [arXiv:hep-th/0612039].

[34] J. Preskill, Magnetic monopoles, Ann. Rev. Nucl. Part. Sci. 34 (1984), 461.

[35] X.G. Wen and E. Witten, Electric and magnetic charges in superstring models, Nucl. Phys. B 261 (1985), 651.

[36] B.R. Greene, D.R. Morrison and C. Vafa, A geometric realization of confinement, Nucl. Phys. B 481 (1996), 513, [arXiv:hep-th/9608039].

[37] H. Verlinde, On metastable branes and a new type of magnetic monopole, arXiv:hep-th/0611069.

[38] O. Aharony, B. Fiol, D. Kutasov and D.A. Sahakyan, Little string theory and heterotic/type II duality, Nucl. Phys. B 679 (2004), 3, [arXiv:hep-th/0310197].

[39] M.B. Green, J.H. Schwarz and L. Brink, $N=4$ Yang-Mills and $N=$ 8 supergravity as limits of string theories, Nucl. Phys. B 198 (1982), 474.

[40] R.R. Metsaev and A.A. Tseytlin, On loop corrections to string theory effective actions, Nucl. Phys. B 298 (1988), 109.

[41] A.A. Tseytlin, On SO(32) heterotic - type I superstring duality in ten dimensions, Phys. Lett. B 367 (1996), 84, [arXiv:hep-th/9510173].

[42] C. Bachas and E. Kiritsis, $F^{4}$ terms in $N=4$ string vacua, Nucl. Phys. Proc. Suppl. 55B (1997), 194, [arXiv:hep-th/9611205].

[43] E. Kiritsis, N.A. Obers and B. Pioline, Heterotic/type II triality and instantons on K3, J. High Energy Phys. 01 (2000), 029, [arXiv:hep-th/0001083].

[44] W. Lerche, On the heterotic/F-theory duality in eight dimensions, arXiv:hep-th/9910207.

[45] A. Clingher, R. Donagi, M. Wijnholt, The Sen Limit, To appear.

[46] C. Bachas, C. Fabre, E. Kiritsis, N.A. Obers and P. Vanhove, Heterotic / type I duality and D-brane instantons, Nucl. Phys. B 509 (1998), 33, [arXiv:hep-th/9707126].

[47] F. Fucito, J.F. Morales and R. Poghossian, Exotic prepotentials from D(-1)D7 dynamics, J. High Energy Phys. 10 (2009), 041, [arXiv:0906.3802 [hep-th]].

[48] D.B. Ray and I.M. Singer, Analytic torsion for complex manifolds, Annals of Mathematics, 2nd Ser., Vol. 98, No. 1. (July 1973), pp. 154177 . 
[49] D.M. Ghilencea, Regularisation techniques for the radiative corrections of the Kaluza-Klein states, Phys. Rev. D 70 (2004), 045011, [arXiv:hep-th/0311187].

[50] M. Bershadsky, S. Cecotti, H. Ooguri and C. Vafa, Kodaira-Spencer theory of gravity and exact results for quantum string amplitudes, Commun. Math. Phys. 165 (1994), 311, [arXiv:hep-th/9309140].

[51] J.-M. Bismut, H. Gillet and C. Soulé, Analytic torsion and holomorphic determinant bundles III: quillen metrics on holomorphic determinants, Comm. Math. Phys. 115 (1988), 302-315.

[52] M.R. Douglas, D.N. Kabat, P. Pouliot and S.H. Shenker, D-branes and short distances in string theory, Nucl. Phys. B 485 (1997), 85, [arXiv:hep-th/9608024].

[53] V. Kaplunovsky and J. Louis, Field dependent gauge couplings in locally supersymmetric effective quantum field theories, Nucl. Phys. B 422 (1994), 57, [arXiv:hep-th/9402005].

[54] V. Kaplunovsky and J. Louis, On Gauge couplings in string theory, Nucl. Phys. B 444 (1995), 191, [arXiv:hep-th/9502077].

[55] J.P. Conlon and E. Palti, On Gauge Threshold corrections for local IIB/F-theory GUTs, arXiv:0907.1362 [hep-th].

[56] K.R. Dienes and A.E. Faraggi, Making ends meet: string unification and low-energy data, Phys. Rev. Lett. 75 (1995), 2646, [arXiv:hep-th/9505018].

[57] Particle data group, Review on Grand Unified Theories.

[58] M.L. Alciati, F. Feruglio, Y. Lin and A. Varagnolo, Proton lifetime from $S U(5)$ unification in extra dimensions, J. High Energy Phys. 03 (2005), 054, [arXiv:hep-ph/0501086].

[59] S. Raby, SUSY GUT Model Building, arXiv:0807.4921 [hep-ph].

[60] T. Goto and T. Nihei, Effect of RRRR dimension five operator on the proton decay in the minimal SU(5) SUGRA GUT model, Phys. Rev. D 59 (1999), 115009, [arXiv:hep-ph/9808255].

[61] H. Murayama and A. Pierce, Not even decoupling can save minimal supersymmetric SU(5), Phys. Rev. D 65 (2002), 055009, [arXiv:hep-ph/0108104].

[62] M. Hortacsu, K.D. Rothe and B. Schroer, Generalized QED in twodimensions and functional determinants, Phys. Rev. D 20 (1979), 3203.

[63] W.I. Weisberger, Normalization of the path integral measure and the coupling constants for bosonic strings, Nucl. Phys. B 284 (1987), 171.

[64] K. Köhler, Equivariant analytic torsion on $P^{n} C$, Math. Ann. 297 (1993), 553-565. 
[65] K. Köhler, Holomorphic torsion on Hermitian symmetric spaces, J. Reine Angew. Math. 460 (1995), 93-116.

[66] V.S. Kaplunovsky, One loop threshold effects in string unification, Nucl. Phys. B 307 (1988), 145, [arXiv:hep-th/9205070].

[67] M. Bershadsky, S. Cecotti, H. Ooguri and C. Vafa, Kodaira-Spencer theory of gravity and exact results for quantum string amplitudes, Commun. Math. Phys. 165 (1994), 311-428, [arXiv: hep-th/9309140].

[68] R. Donagi, S. Katz and M. Wijnholt, Weak coupling, degeneration and log Calabi-Yau spaces, To appear.

[69] R. Blumenhagen, gauge coupling unification in f-theory grand unified theories, Phys. Rev. Lett. 102 (2009), 071601, [arXiv:0812.0248 [hep-th]].

[70] R. Donagi and M. Wijnholt, Gluing Branes, I, [arXiv:1104.2610 [hep-th]].

[71] R. Donagi and M. Wijnholt, Gluing Branes II: Flavour Physics and String Duality, arXiv:1112.4854 [hep-th].

[72] N. Akerblom, R. Blumenhagen, D. Lust and M. Schmidt-Sommerfeld, Thresholds for intersecting D-branes revisited, Phys. Lett. B 652 (2007), 53, [arXiv:0705.2150 [hep-th]].

[73] P. Nath and P. Fileviez Perez, Proton stability in grand unified theories, in strings, and in branes, Phys. Rep. 441 (2007), 191, [arXiv:hep-ph/0601023].

[74] S. Raby, Proton decay, arXiv:hep-ph/0211024.

[75] R. Donagi, Spectral Covers, [arXiv:alg-geom/9505009].

[76] G. Curio and R.Y. Donagi, Moduli in $N=1$ heterotic/F-theory duality, Nucl. Phys. B 518 (1998), 603, [arXiv:hep-th/9801057].

[77] R. Tatar and T. Watari, Proton decay, Yukawa couplings and underlying gauge symmetry in string theory, Nucl. Phys. B 747 (2006), 212, [arXiv:hep-th/0602238].

[78] E. Witten, Anomaly cancellation on G(2) manifolds, arXiv:hep-th/ 0108165.

[79] R. Donagi and M. Wijnholt, MSW Instantons, arXiv:1005.5391 [hep-th].

[80] R. Blumenhagen, S. Moster and E. Plauschinn, Moduli stabilisation versus chirality for MSSM like type IIB orientifolds, J. High Energy Phys. 01 (2008), 058, [arXiv:0711.3389 [hep-th]].

[81] J.C. Pati, The essential role of string-derived symmetries in ensuring proton stability and light neutrino masses, Phys. Lett. B 388 (1996), 532, [arXiv:hep-ph/9607446]. 
[82] M. Wijnholt, F-Theory, GUTs and Chiral Matter, arXiv:0809.3878 [hep-th].

[83] version 3 of reference [21].

[84] L.J. Hall and U. Sarid, Gravitational smearing of minimal supersymmetric unification predictions, Phys. Rev. Lett. 70 (1993), 2673, [arXiv:hep-ph/9210240].

[85] J. Polchinski, String theory. vol. 2: superstring theory and beyond, Cambridge, UK, University Press, 1998, 531 p.

[86] C. Bachas, C. Fabre, E. Kiritsis, N.A. Obers and P. Vanhove, Heterotic/type-I duality and D-brane instantons, Nucl. Phys. B 509 (1998), 33, [arXiv:hep-th/9707126].

[87] C. Bachas and E. Kiritsis, $F^{4}$ terms in $N=4$ string vacua, Nucl. Phys. Proc. Suppl. 55B (1997), 194, [arXiv:hep-th/9611205].

[88] E. Witten, Chern-Simons gauge theory as a string theory, Prog. Math. 133 (1995), 637, [arXiv:hep-th/9207094].

[89] S.A. Merkulov, Strongly homotopy algebras of a Kähler manifold, Internat. Math. Res. Notices no.3 (1999), 153-164, [arXiv.org:math/ 9809172]. 
